\title{
Mach Reflection of Cellular Detonations
}

\author{
Yannick Fortin \\ Master of Engineering \\ Department of Mechanical Engineering \\ McGill University \\ Montréal,Québec \\ 2014-07-08

\begin{abstract}
A thesis submitted to McGill Master of Engineering
\end{abstract} \\ University in partial fulfillment of \\ the requirements of the degree of \\ (c) Y. Fortin 2014
}




\section{TABLE OF CONTENTS}

LIST OF TABLES $\ldots \ldots \ldots \ldots \ldots \ldots \ldots \ldots \ldots$ iv

LIST OF FIGURES $\ldots \ldots \ldots \ldots \ldots \ldots \ldots$. . . . . . . . . . . . . .

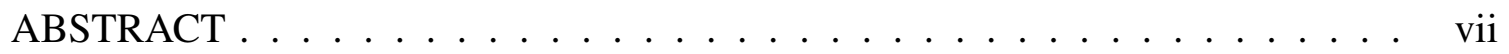

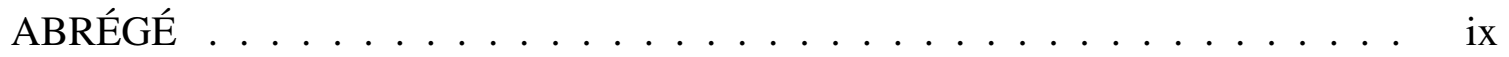

ACKNOWLEDGEMENTS . . . . . . . . . . . . . . . . . . . xi

1 Introduction $\ldots \ldots \ldots \ldots \ldots \ldots \ldots \ldots \ldots$

$1.1 \quad$ Background $\ldots \ldots \ldots \ldots \ldots \ldots \ldots \ldots$

$1.2 \quad$ Past Studies . . . . . . . . . . . . . . . . . . . . . . . . . . 8

1.3 Three-Shock Theory Limitations $\ldots \ldots \ldots$

1.4 Motivation . . . . . . . . . . . . . . . . . . . . . . . . 19

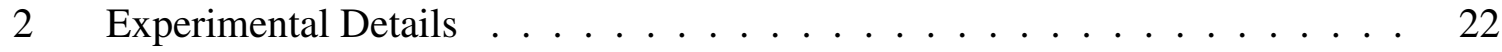

$2.1 \quad$ Experimental setup . . . . . . . . . . . . . . . . . 22

$2.2 \quad$ Mixture Selection and Preparation $\ldots \ldots \ldots \ldots \ldots$

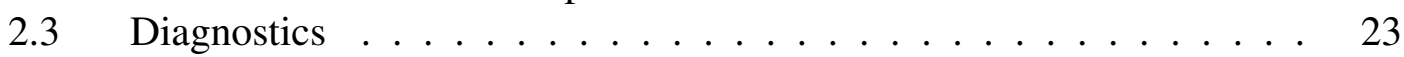

$3 \quad$ Results and Discussion $\ldots \ldots \ldots \ldots \ldots$. . . . . . . . . . . . . 25

$3.1 \quad$ Effect of Initial Pressure. . . . . . . . . . . . . . . . . . . . . . . 25

3.2 Effect of Wedge Angle . . . . . . . . . . . . . . . . . . 27

3.3 Effect of Mixture . . . . . . . . . . . . . . . . . . . . . 30

3.4 Critical Angle for Transition $\ldots \ldots \ldots \ldots \ldots$

3.5 Smoke Foils . . . . . . . . . . . . . . . . . . . . . . 37

$3.6 \quad$ Graphical Results . . . . . . . . . . . . . . . . . . . . 40

3.6 .1 Effect of Initial Pressure. . . . . . . . . . . . . . . . . . . 40

3.6 .2 Effect of Wedge Angle and Mixture. . . . . . . . . . . . 44

3.6 .3 Smoke foils VS schlieren $\ldots \ldots \ldots \ldots \ldots$ 


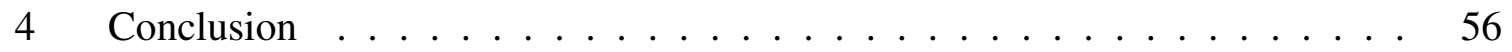

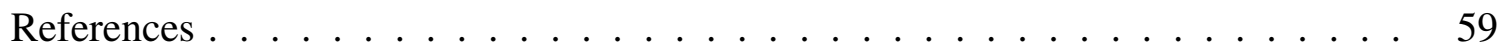

A $\quad$ schlieren Setup $\ldots \ldots \ldots \ldots$. . . . . . . . . . . . . . . . 62

B Theory . . . . . . . . . . . . . . . . . . . . 72 
Table

LIST OF TABLES

page 


\section{LIST OF FIGURES}

$1-1$ Shock Reflection $\ldots \ldots \ldots \ldots$. . . . . . . . . . . . . . 1

1-2 Diagram of regular reflection sitting on the triple point . . . . . . . . . 2

1-3 Polar diagram of regular reflection . . . . . . . . . . . . 3

$1-4$ Critical angle . . . . . . . . . . . . . . . 4

1-5 Diagram of Mach reflection sitting on the triple point $\ldots \ldots \ldots$

1-6 Polar diagram of Mach Reflection . . . . . . . . . . . . . . . 6

1-7 Mach Reflection Photograph . . . . . . . . . . . . . . . . . 8

1-8 Relationship between Triple point trajectory and wedge angle . . . . . . . 18

1-9 Reactive Three-shock theory for various dilution ratios of $\mathrm{C}_{2} \mathrm{H}_{2}+2.5 \mathrm{O}_{2}$

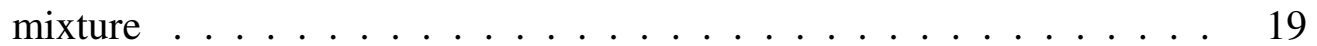

$2-1 \quad$ Schematic of the windowed section . . . . . . . . . . . . . 22

$3-1 \quad \mathrm{C}_{2} \mathrm{H}_{2}+2.5 \mathrm{O}_{2}+70 \%$ Ar $30^{\circ}$ wedge . . . . . . . . . . . . 26

$3-2 \mathrm{C}_{2} \mathrm{H}_{2}+2.5 \mathrm{O}_{2}+70 \%$ Ar $30^{\circ}$ wedge . . . . . . . . . . . 27

$3-3 \quad \mathrm{C}_{2} \mathrm{H}_{2}+2.5 \mathrm{O}_{2}+70 \%$ Ar $10^{\circ}$ wedge . . . . . . . . . . . . 28

$3-4 \quad \mathrm{C}_{2} \mathrm{H}_{2}+2.5 \mathrm{O}_{2}+70 \%$ Ar $35^{\circ}$ wedge . . . . . . . . . . . . 29

$3-5 \mathrm{C}_{3} \mathrm{H}_{8}+5 \mathrm{O}_{2} 10^{\circ}$ wedge $\ldots \ldots \ldots \ldots$

$3-6 \mathrm{C}_{3} \mathrm{H}_{8}+5 \mathrm{O}_{2} 35^{\circ}$ wedge $\ldots \ldots \ldots \ldots \ldots$

$3-7 \quad \mathrm{C}_{2} \mathrm{H}_{2}+2.5 \mathrm{O}_{2} 10^{\circ}$ wedge $\ldots \ldots \ldots \ldots$

$3-8 \mathrm{C}_{2} \mathrm{H}_{2}+2.5 \mathrm{O}_{2} 35^{\circ}$ wedge $\ldots \ldots \ldots \ldots$ 


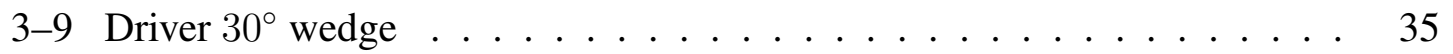

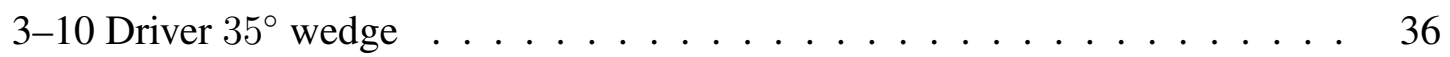

3-11 Driver $35^{\circ}$ wedge $\ldots \ldots \ldots \ldots \ldots \ldots \ldots \ldots \ldots \ldots$

3-12 Smoke foils of a detonation in $\mathrm{C}_{2} \mathrm{H}_{2}+2.5 \mathrm{O}_{2}+70 \% \mathrm{Ar}$ at $5 \mathrm{kPa}$ over a $30^{\circ} \quad 38$

3-13 Smoke foils of a detonations in $\mathrm{C}_{3} \mathrm{H}_{8}+5 \mathrm{O}_{2}$ at $5 \mathrm{kPa}$ over a $30^{\circ}$ wedge . . $\quad 39$

3-14 Height of Mach stem VS propagation distance for detonation waves in mixtures of a) $\mathrm{C}_{2} \mathrm{H}_{2}+2.5 \mathrm{O}_{2}+70 \% \mathrm{Ar}$ b) $\mathrm{C}_{3} \mathrm{H}_{8}+5 \mathrm{O}_{2}$ and c) $\mathrm{C}_{2} \mathrm{H}_{2}+$ $2.5 \mathrm{O}_{2}$ at different initial pressures, over a $30^{\circ}$ wedge $\ldots \ldots \ldots .42$

3-15 Height of Mach stem VS propagation distance for $\mathrm{C}_{2} \mathrm{H}_{2}+2.5 \mathrm{O}_{2}+70 \% \mathrm{Ar}$ at $20 \mathrm{kPa}$ over different wedges $\ldots \ldots \ldots \ldots \ldots$

3-16 Height of Mach stem VS propagation distance for $\mathrm{C}_{3} \mathrm{H}_{8}+5 \mathrm{O}_{2}$ at $10 \mathrm{kPa}$ over different wedges $\ldots \ldots \ldots \ldots$. . . . . . . . . . . . . . 49

3-17 Height of Mach stem VS propagation distance for $\mathrm{C}_{2} \mathrm{H}_{2}+2.5 \mathrm{O}_{2}$ at $20 \mathrm{kPa}$ over different wedges $\ldots \ldots \ldots \ldots \ldots \ldots \ldots \ldots$

3-18 Triple point height vs distance traveled over a $30^{\circ}$ wedge for a) $\mathrm{C}_{2} \mathrm{H}_{2}+$ $\left.2.5 \mathrm{O}_{2}+70 \% \mathrm{Ar} \mathrm{P}_{\text {ini }}=20 \mathrm{kPa} \mathrm{b}\right) \mathrm{C}_{2} \mathrm{H}_{2}+2.5 \mathrm{O}_{2}+70 \% \mathrm{Ar} \mathrm{P}_{\text {ini }}=5 \mathrm{kPa}$ c) $\left.\mathrm{C}_{3} \mathrm{H}_{8}+5 \mathrm{O}_{2} \mathrm{P}_{\text {ini }}=10 \mathrm{kPa}, \mathrm{d}\right) \mathrm{C}_{3} \mathrm{H}_{8}+5 \mathrm{O}_{2} \mathrm{P}_{\text {ini }}=5 \mathrm{kPa} \ldots \ldots \ldots 55$

A-1 Simple schlieren a), b)with point light source and c), d) extended light source 63

A-2 Z-type schlieren configuration used . . . . . . . . . . . . . 65

A-3 Condenser lens . . . . . . . . . . . . . . . 66

A-4 Photodiode triggering circuit $\ldots \ldots \ldots \ldots \ldots$. . . . . 70

A-5 Spherical shock generated by high voltage discharge obtained from Grondin $[11] \ldots \ldots \ldots \ldots \ldots \ldots \ldots \ldots$

B-1 Oblique Detonation Diagram $\ldots \ldots \ldots \ldots \ldots . \ldots \ldots$

B-2 Three-Shock configuration from the triple point . . . . . . . . . 74 


\begin{abstract}
In this thesis the Mach reflection of cellular detonation was studied. The detonations were propagating in mixtures of $\mathrm{C}_{2} \mathrm{H}_{2}+2.5 \mathrm{O}_{2}, \mathrm{C}_{2} \mathrm{H}_{2}+2.5 \mathrm{O}_{2}+70 \% \mathrm{Ar}$ and $\mathrm{C}_{3} \mathrm{H}_{8}+5 \mathrm{O}_{2}$ at initial pressures of $3 \mathrm{kPa} \leq \mathrm{P}_{o} \leq 20 \mathrm{kPa}$. Wedge angles of $10^{\circ}, 20^{\circ}, 30^{\circ}, 35^{\circ}$ and $40^{\circ}$ were used. The Mach reflection was observed by schlieren photography and smoked foils. From the results it is seen that for small wedge angles (e.g. $\theta_{w}=10^{\circ}$ ), the Mach stem is curved and blends smoothly with the incident detonation front with no discernible triple point. For larger wedge angles the typical features of the Mach reflection phenomenon can be observed. A sharp "kink" between the Mach stem and the incident detonation also gives a more definite location of the triple point. Based on the measurements of the variation of the height of the Mach stem versus the incident wave propagation it was found that the triple point boundary between the Mach stem and the incident wave follows a curved line in contrast to a straight line trajectory of the triple point obtained via regular three-shock theory. The observed critical angle for the transition between regular and Mach reflection does not agree with the predictions using the self-similar three-shock theory except for the $\mathrm{C}_{2} \mathrm{H}_{2}+2.5 \mathrm{O}_{2}$ mixture. Schlieren photographs and smoke foils illustrate the transition of the cellular structure from the overdriven Mach stem to the incident detonation indicating that a description of the triple point region requires a consideration between the interaction of the transverse waves of cellular instability and the reflected shock of the Mach reflection process. A dependence of the Mach stem height on cell size and initial pressure were also observed. It may be concluded that Mach reflections of cellular detonations cannot be described by simple self-similar theory and would require the description of the transient
\end{abstract}


propagation of cellular detonation under the change in the boundary condition of diffraction by a wedge. 


\begin{abstract}
ABRÉGÉ
Une étude de la réflexion de Mach sur des détonations cellulaires à été fait dans ce mémoire. Les détonations sont propagées dans des mixtures de $\mathrm{C}_{2} \mathrm{H}_{2}+2.5 \mathrm{O}_{2}, \mathrm{C}_{2} \mathrm{H}_{2}+$ $2.5 \mathrm{O}_{2}+70 \%$ Ar et $\mathrm{C}_{3} \mathrm{H}_{8}+5 \mathrm{O}_{2}$ pour des pressions initiales de $3 \mathrm{kPa} \leq \mathrm{P}_{o} \leq 20 \mathrm{kPa}$. Des coins aux angles de $10^{\circ}, 20^{\circ}, 30^{\circ}, 35^{\circ}$ et $40^{\circ}$ ont été utilisé. Les réflexions de Mach sont observés par des photos schlieren et des feuilles fumées. D'après les résultats, il est observé que pour des coins à petit angle (e.g. $\theta_{w}=10^{\circ}$ ), la souche de Mach courbe et ce mélange doucement avec le front de la détonation incidente sans observation de point triple. Pour les coins aux angles plus grands les attributs typiques du phénom ène de réflexion de Mach sont observés. Un point de rebroussement clair entre la souche de Mach et la détonation incidente procure un point triple mieux localisé. Basé sur des mesures de la variation de hauteur de la souche de Mach sur la surface des coins il a été démontré que la limite du point triple entra la souche de Mach et la vague incidente suis une ligne courbé en contraste avec la trajectoire de ligne droite obtenu avec la théorie de trois choc. Langle critique observé entre la réflexion réguli ére et la réflexion de Mach nest pas en accord avec les prédiction de la théorie auto-similaire des trois chocs sauf pour la mixture de $\mathrm{C}_{2} \mathrm{H}_{2}+$ $2.5 \mathrm{O}_{2}$. Les photos schlieren et les feuilles fumées illustre la transition de la structure cellulaire entre la souche de Mach et la détonation incidente qui indique que la description de la région du point triple requiert une considération entre linteraction des vague transverse de linstabilit'e cellulaire ainsi que londe reflété du processus de réflexion. Une dépendance de la hauteur de la souche de Mach sur la taille des cellules et pression initial ont été observé. Il peut être conclût que la réflexion de Mach pour les détonations cellulaires ne peut pas
\end{abstract}


être décrite par une simple théorie auto-similaire et quune description des propagations transientes des détonations cellulaires affectés par le changement de condition de la limite de bordure causé par une diffraction par un coin est nécessaire. 


\section{ACKNOWLEDGEMENTS}

First and foremost I would like thank my supervisor Professor John H.S. Lee, who has always given me insight and support in dealing with problems both in the laboratory and in life. He has proven to be a mentor in many things. His healthy criticism of my work has made my work experience much more valuable. His patience in teaching things in a simple manner has also tremendously helped my understanding of the subject. I also thank the rest of the Shock Wave Physics Group past and present. Amanda Starr, Adam Smith, Anne Jesuthasan deserve special thanks. Because of them the work environment was much more enjoyable especially while dealing with forced experimental downtime. Thanks to Liu Jie who has been invaluable help by carrying out some experiments. He has brought fresh ideas to the problem and helped streamlining experimental procedure by updating the equipment. Sincere thanks are due also to Jean-Sebastien Grondin, whom has initiated me in the work environment with patience and smile on a daily basis thus fueling my desire to pursue my studies. He provided close cooperation and much technical expertise in the experimental schlieren setup required for my experiments. The experimental setup could not be what it is without the tremendous work by the Machine Tool Lab technicians whom have help in the building of most of my setup as well as provided help in the maintenance process. Special thanks are due to Tony Micozzi, Andreas Hofmann and Gary Savard for going out of their way in helping me. The present work could not be completed without the unending support of my parents and my brother. Thank you for all the positive reenforcement you all have provided in some of the darkest times. To all my best friends whom have made it mandatory to meet once a week to keep me smiling and providing a 
fun environment out of the Lab. Last but not least, I would like to thank Leakena Mom, my love, whom has been with me through the whole process. Helping me in my downtimes and inspiring me to go above and beyond myself. 


\section{CHAPTER 1}

\section{Introduction}

\subsection{Background}

When a shockwave propagates up a wedge the incident shock is reflected. There are two basic types of shock reflections possible, a regular reflection and irregular reflection. Mach reflection being a special case of irregular reflection. Both regular and Mach reflection are illustrated in Fig. 1-1(a) and 1-1(b) respectively.

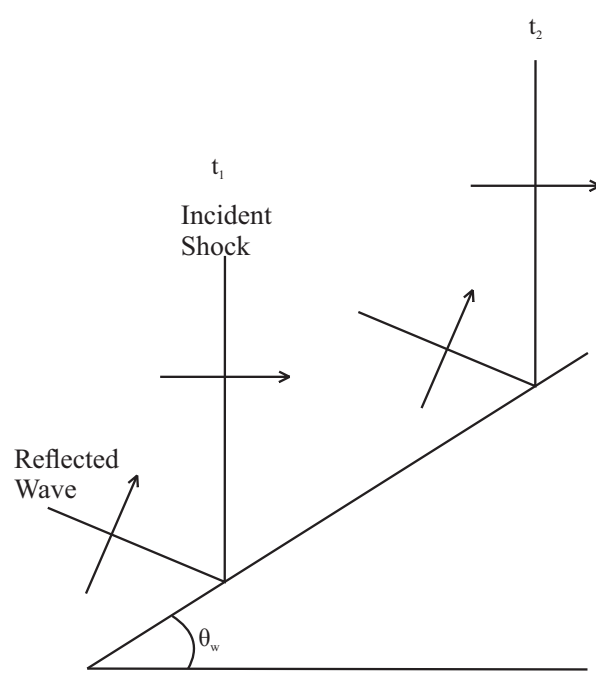

(a) Regular Reflection

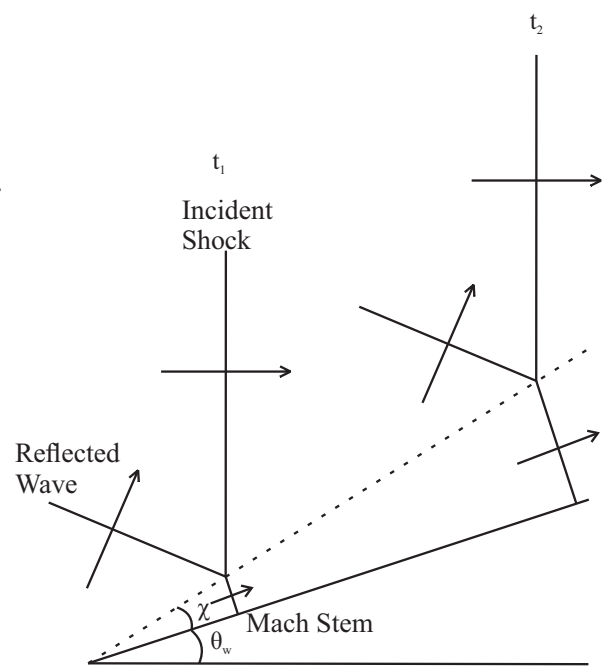

(b) Mach Reflection

Figure 1-1: Diagram of Shock reflection at various times

For regular reflection the intersection of the incident and reflected shock is on the wedge surface whereas for Mach reflection there are three shocks; the incident, reflected 
and a third shock called the Mach stem. The shock intersection point is called the triple point and it travels up from the wedge surface. Whether regular or Mach reflection occurs depends on the angle of the wedge. It is of interest to find the wedge angle at which the transition between regular reflection and Mach reflection occurs. To find this critical angle of transition it is more convenient to consider a frame of reference at the shock intersection as shown in Fig. 1-2.

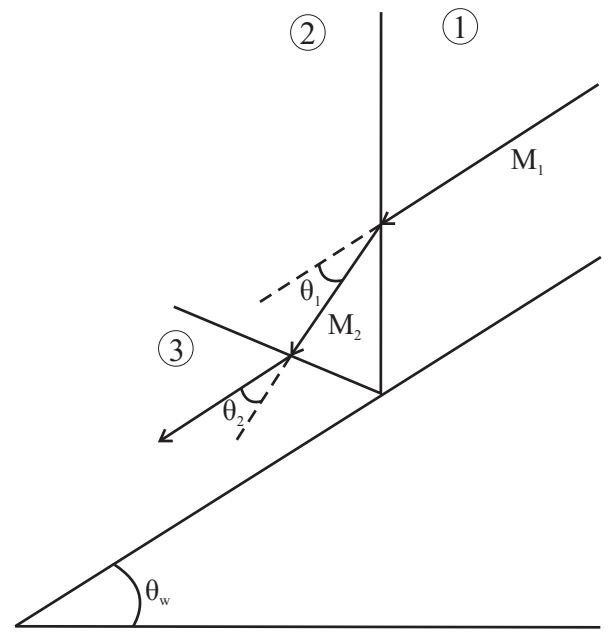

Figure 1-2: Diagram of regular reflection sitting on the triple point

For the regular reflection the flow comes in parallel to the wedge and it is deflected by the incident shock by an angle $\theta_{1}$ and then the reflected wave bends the flow back by $\theta_{2}$ such that it returns parallel to the wedge with a total deflection $\theta_{t o t}=\theta_{1}+\theta_{2}=0$. The shock polar for regular reflection is shown in Fig. 1-3. 


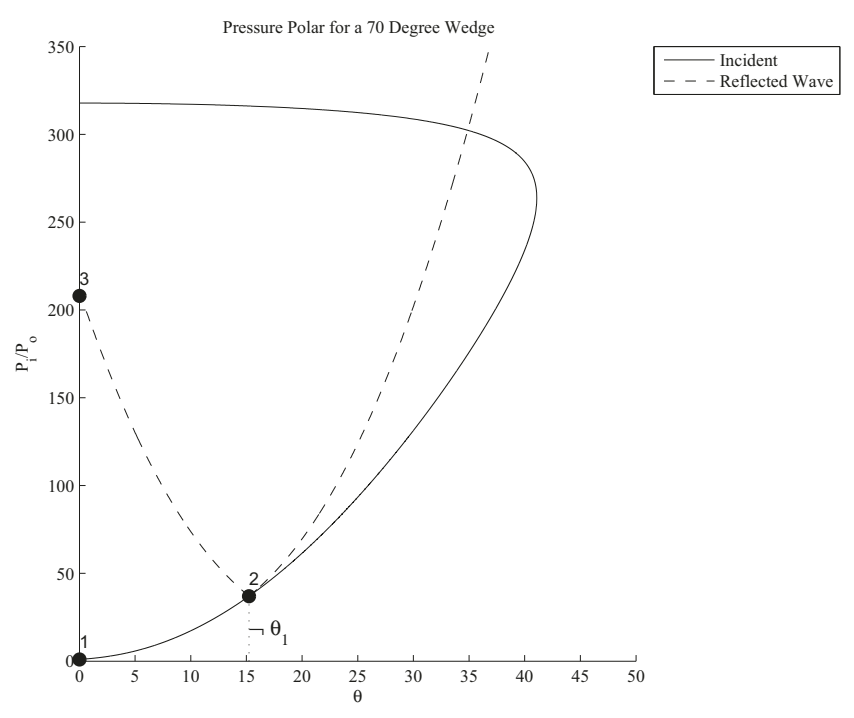

Figure 1-3: Polar diagram of regular reflection

The pressure in region 3 can be solved using shock polars for the incident and the reflected wave. If $\theta_{t o t}=0$ then the reflected polar muxt intersect the y-axis. As the wedge angle is decreased the reflected shock polar will be shifted along the incident shock polar and its magnitude will decrease since the reflected wave becomes weaker for a smaller wedge angle. There will be a limiting angle at which the reflected wave polar will just become tangent to the y-axis. This angle is the minimum wedge angle at which regular reflection can be observed as shown in Fig. 1-4(a). Below that angle the reflected wave polar will not intersect the y-axis, as seen in Fig. 1-4(b). This means that the flow behind the reflected wave is no longer parallel to the wedge surface as the maximum deflection angle that can be acheived by the reflected wave $\theta_{2 \max }$ is smaller than $\theta_{1}$. It is not possible to solve for $\theta_{2}$ unless we introduce a third shock (Mach stem) as illustrated in Fig. 1-5 which shows the flow with respect to a frame of reference travelling with the triple point. 


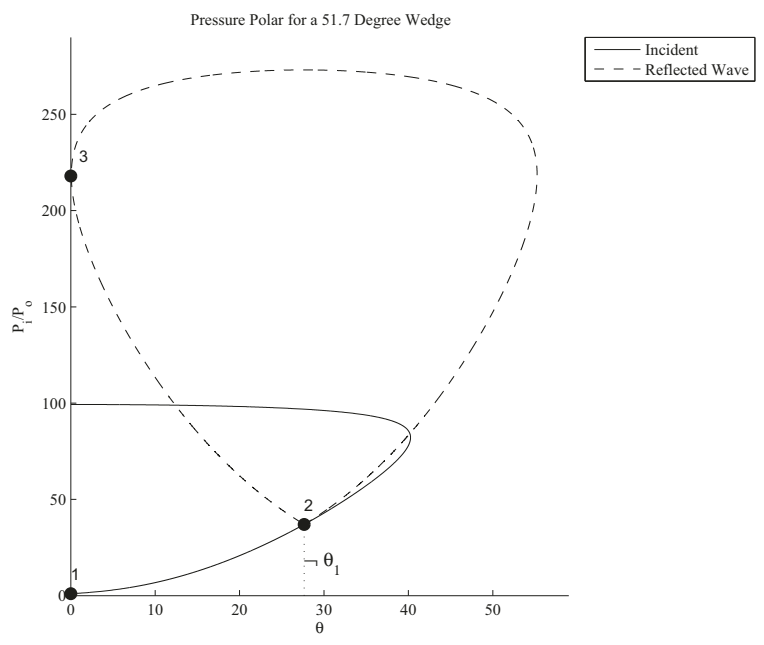

(a) Critical Wedge angle

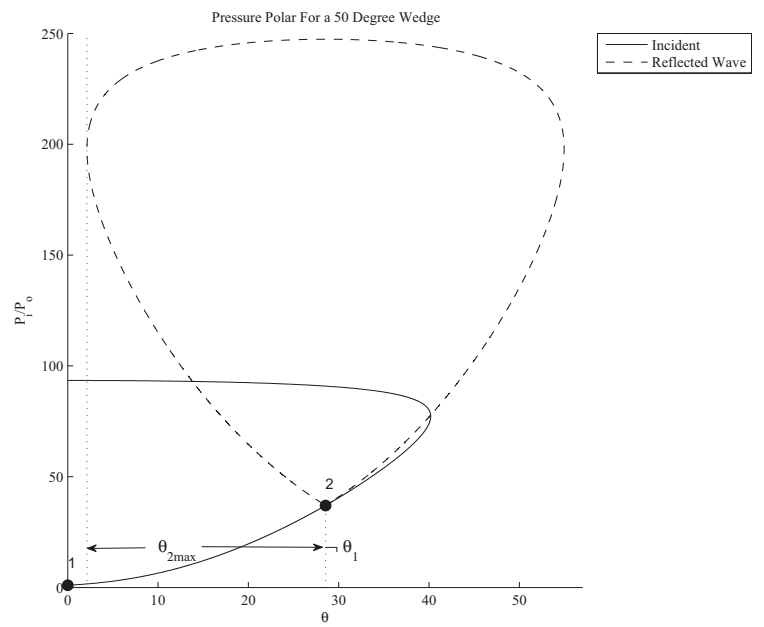

(b) Wedge angle below critical angle

Figure 1-4: Graphical representation of a) Minimum Wedge angle for observable regular reflection b) Below critical angle 


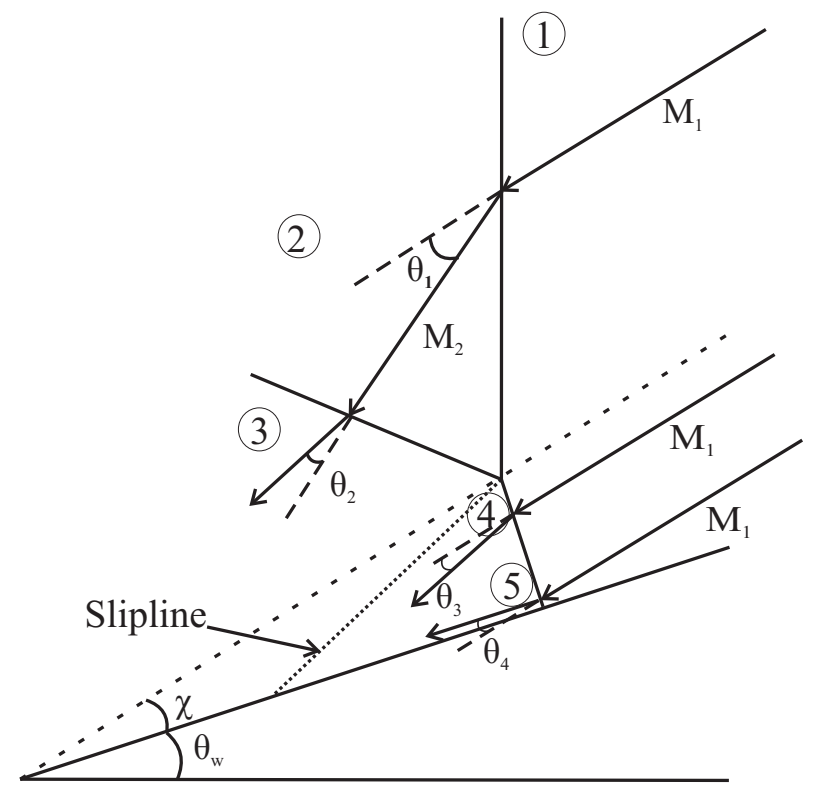

Figure 1-5: Diagram of Mach reflection sitting on the triple point

The density in region 3 is not the same as the density in region 4 because the gas in the region 3 has been subjected to two shockwaves, while region 4 only passed through one shock. Therefore there is a discontinuity in density (and temperature) between the two regions. However the streamlines are parallel thus we denote the discontinuity as a slipline. Across the slipline the pressure must be the same. Since the pressure in region 4 must lie on the incident polar, because the incoming flow across the Mach stem is $\mathrm{M}_{1}$, and the pressure in region 3 must be on the reflected wave polar the solution is thus at the intersection of the two shock polars as shown in Fig.(1-6). 


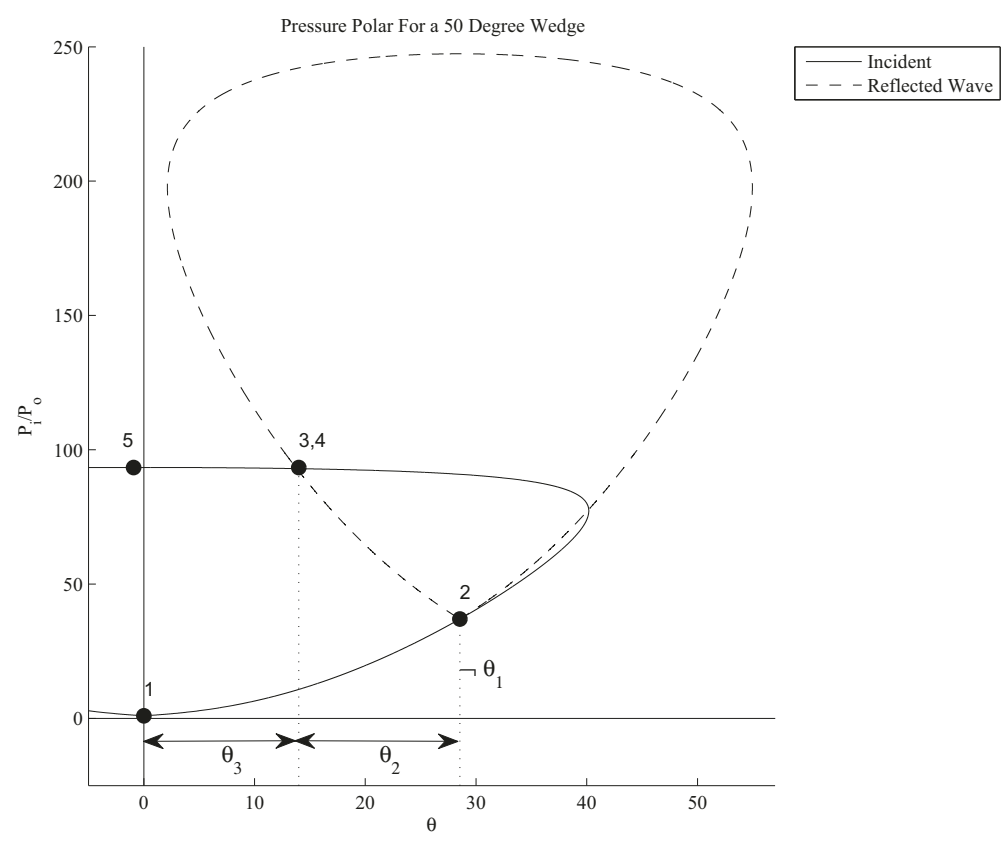

Figure 1-6: Polar diagram of Mach Reflection

With points 3 and 4 at the intersection of the two shock polars, the deflection of the streamlines $\theta_{2}$ and $\theta_{3}$ can be obtained and the trajectory of the triple point angle $\chi$ can be found. This was first analyzed by von Neumann [32] in 1943 and later referred to as the Three-shock Theory. More details on the application of this method can be found in appendix B.

The Mach Stem cannot be straight since the streamlines in region 4 are not parallel to the wedge surface, however to satisfy the boundary conditions the streamlines in region 5 must be parallel to the surface. Since both regions have the same incoming flow $\mathrm{M}_{1}$, they must lie on the incident polar, but their deflections are not the same, thus we conclude 
that the wave angle varies along the Mach stem to accommodate the flow deflection at the boundary.

Shock waves are very thin. Detonation waves can be aproximated as shockwaves with energy release at the front. They have a finite thickness that is at least one cell length. The consequence of this is that the problem can no longer be considered as quasi-steady. Therefore the three-shock theory, that solves the flow angles behind the Mach reflection based on a reference frame situated on the triple point, can no longer be used. However both shock and detonation waves have Mach reflection and both share similarities as shown in Fig. 1-7. Both have a perpendicular incident wave at the channel wall and curved reflected waves. But several features are different. The triple point is usually well defined for shock waves but in the case of detonation waves it becomes a small region near the intersection pattern with the incident wave rather than a sharp point. The reflected wave can sometimes be difficult to define due to the flowfield behind the incident detonation wave masking the weak density gradients across it. The Mach stem being overdriven leads to the cell sizes being smaller, creating a different structure between it and the incident wave. This permits the triple point region to be localized even if the reflected wave is not visible. This topic has been the center of many investigations in the past, but several key problems remain unresolved. 


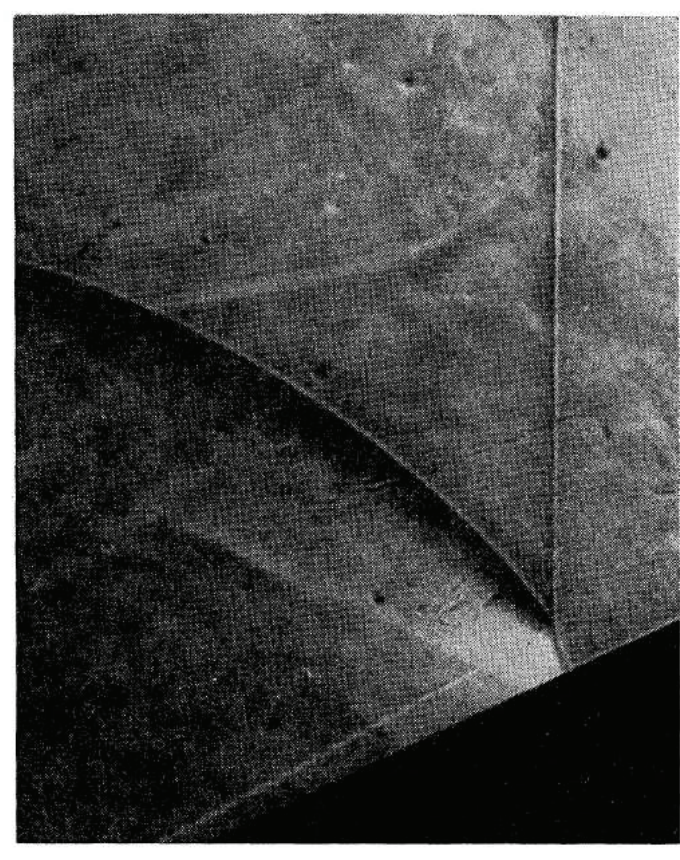

a)

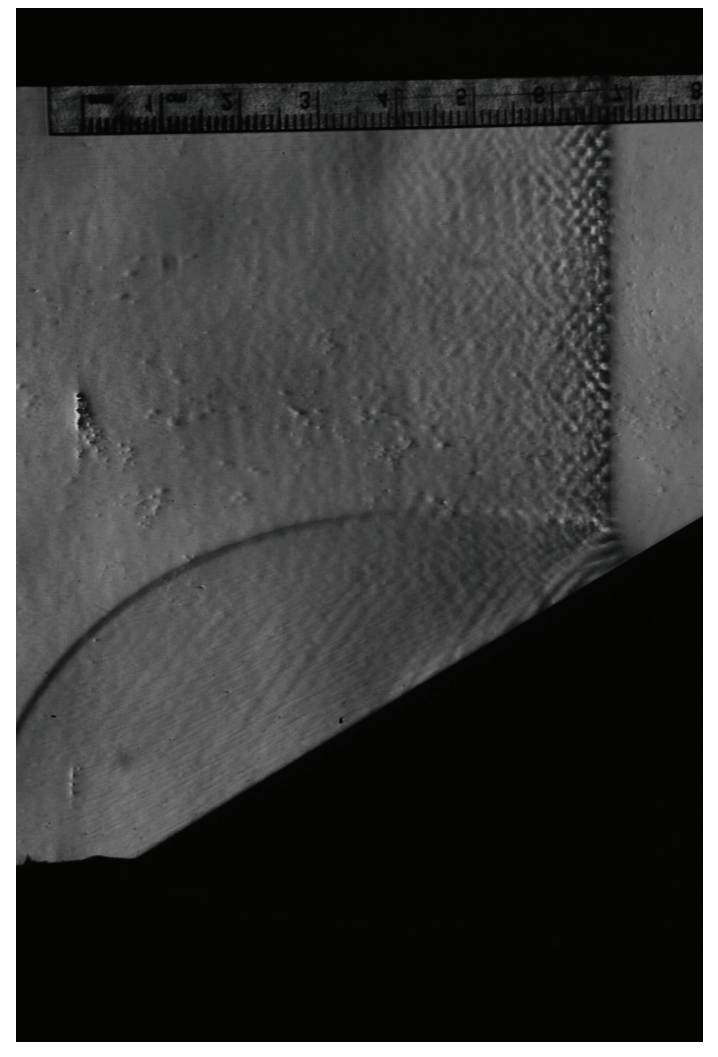

b)

Figure 1-7: schlieren photograph of Mach reflection of a) shockwave(Bleakney et al. [4]) and b) detonation wave

\subsection{Past Studies}

R.S. Ong (1955) [26] studied planar Chapman ChapmanJouguet (CJ) detonation in stoichiometric hydrogen-oxygen mixtures, at initial pressure 20psia, reflecting from wedges varying between $0^{\circ}$ and $45^{\circ}$. From schlieren photographs he measured the trajectory of the triple point and compared the experimental results with theoretical calculations. By assuming that the phenomenon is self-similar he reduced the problem to a time-independent boundary value problem in a pseudo-stationary frame with coordinates $\eta$ and $\zeta$ where $\eta$ is 
$\mathrm{x} / \mathrm{t}$ and $\zeta$ is $\mathrm{y} / \mathrm{t}$. He treated the detonation wave as a thin discontinuity with a heat release and assumed that the reflected shock is circular. This assumption allowed him to linearize the differential equations of motion. Using the circular reflected wave as the zeroth order solution he adjusted the shape by adding a small perturbation function to the derivatives of the flow parameters and calculated the final shape of the reflected shock. He obtained very good agreement between calculations and experiments up to a $15^{\circ}$ wedge after which the calculation started to overpredict by $15 \%$ or more. He also calculated the triple point trajectory and compared it with the measured trajectory from the experiments and obtained a very good agreement (within $0.5^{\circ}$ ) up to a $30^{\circ}$ wedge. He did not report the calculated angle for the $35^{\circ}$ wedge and the photographs of the $45^{\circ}$ wedge show regular reflection.

Gvozderva and Predvoditeleva (1969) [14] investigated Mach reflections of detonations in methane-oxygen and hydrogen-oxygen mixtures at 1atm propagating along 2 wedges of $20^{\circ}$ and $35^{\circ}$ only. They measured the triple point trajectory from schlieren photographs and compared their results with the three shock theory. They found that the calculations underpredicted the trajectory by $4^{\circ}$ for the $20^{\circ}$ wedge and they have obtained Mach reflection for the $35^{\circ}$ while the theory predicted regular reflection. They pointed out that the $35^{\circ}$ wedge photographs indicate a possibility of more complex Mach reflections.

Gavrilenko et al. (1980) [10] studied "supercompressed" detonations in equimolar and stoichiometric acetylene-oxygen mixtures at 1atm. The detonations were supercompressed by propagating through a rectangular channel whose area was decreased with symmetrical wedge inserts varying from $15^{\circ}$ to $90^{\circ}$. The velocity of the detonation was measured by streak photography. The supercompression ratio as a function of wedge angle was obtained. They found that the maximum supercompression for both mixtures occured 
when $30^{\circ}$ wedge inserts were used to reduce the channel area 5 times the original area. He noticed that the theoretical critical wedge angle for transition between Mach reflection and regular reflection was $34^{\circ}$ for both mixtures and proceeded to make theoritical calculations for the critical angle for mixtures of $2 \mathrm{H}_{2}+\mathrm{O}_{2}, \mathrm{H}_{2}+\mathrm{O}_{2}, 4 \mathrm{H}_{2}+\mathrm{O}_{2}, \mathrm{C}_{2} \mathrm{H}_{2}+2.5 \mathrm{O}_{2}, \mathrm{C}_{2} \mathrm{H}_{2}+$ air (stochiometric), $\mathrm{CO}+2 \mathrm{O}_{2}$ and $\mathrm{CH}_{4}+$ air (stoichiometric) at varying pressures from 0.1 to $1 \mathrm{~atm}$. He found that the theoretical critical wedge angle is nearly independent on the mixture as well as initial pressure with a calculated critical angle of $34 \pm 0.4^{\circ}$ for all undiluted mixtures. However dilution with argon increased the theoretical critical angle by up to $3^{\circ}$.

Gavrilenko and Prokhorov (1982-1983) [9], [8] studied Mach reflections of detonations in stoichiometric mixtures of acetylene-oxygen and hydrogen-oxygen at initial pressures varying from 0.05 to $1 \mathrm{~atm}$. They measured the triple point trajectory and critical angle using smoke foils. They report a single critical angle of $40 \pm 1^{\circ}$ for both mixtures which is $6^{\circ}$ above the calculated theoretical critical angle, however no mention was made about the initial pressure at which this critical angle was found nor are there any mention of the effects of different initial pressures on the critical angle. The smoke foils showed that the triple point trajectory was not self-similar as in the case of shockwaves. They believed that that non self-similarity was due to the interaction between the large cells in the incident wave with the triple-points.

Edwards et al. (1984) [7] investigated detonation diffraction in mixtures of hydrogenoxygen and acetylene-oxygen with various degrees of dilution. The triple point trajectory was measured from schlieren photographs and used to compute the degree of overdrive of the Mach stem. Smoke foils were used to determine the cell sizes behind the Mach stem and he compared with Barthels acoustic theory for predicting wave spacing. He found 
that up to an overdrive factor of 1.2 Barthels theory compares well with experiment but beyond this value the theory overpredicts the wave spacing by $15 \%$. No comparison was made with the three shock theory. He also found from the schlieren photographs that the triple point trajectory was smaller when the cell size of a given mixture was smaller but does not elaborate further.

Nettleton (1987) [23] compared the results of Edwards with theoretical calculations from both the three shock and Whithams ray shock theory [34]. Ray shock theory is an area-Mach number relationship developed for shockwaves. He assumed the detonations to be strong shockwaves followed by a large induction zone and solved theoretically for the critical angle of transition using three shock theory. He calculated a critical angle of $65^{\circ}$ which is $17^{\circ}$ higher than the experimental value of $48 \pm 2^{\circ}$ reported by Edwards. Both Ray shock and three shock theory yielded similar results for the triple point trajectory but overpredicted results from schlieren photographs by $5^{\circ}-12^{\circ}$ and $3^{\circ}-8^{\circ}$ for smoke foils.

Meltzer(1993) [21] studied detonation reflection in stoichiometric hydrogen-oxygen at an initial pressure of $20 \mathrm{kPa}$ propagating over wedges varying between $10^{\circ}-50^{\circ}$ in $5^{\circ}$ increments. The triple point trajectory and the critical angle were measured from smoke foils by looking for overdriven cells on the foils and he assumed they were generated by the Mach stem and drawing a straight line to the apex between these two regions. This proved to be difficult for the smaller wedges (below $20^{\circ}$ ) as the cell size ratio $\lambda_{\text {over }} / \lambda$ varies between $0.58-0.82$, thus resulting in a large scatter in experimental data. Although this method was deemed more accurate for the larger wedges the results still compared poorly with the prediction from three-shock theory which underpredicts the trajectory by $6^{\circ}-10^{\circ}$. The reported critical angle is between $40^{\circ}-45^{\circ}$. They claimed that the poor agreement was 
due to the inaccuracy of the measurements techniques and that the effects of the transverse waves, which would contribute to loss terms, are not accounted for.

Akbar (1997) [1] investigated the Mach reflection of detonations in hydrogren-oxygen and acetylene-oxygen mixtures with various degrees of dilutions, over wedges ranging from $15^{\circ}-45^{\circ}$ as well as half cylinder obstacles. Using shadowgraph pictures and smoke foils he measured the triple point trajectory by drawing a straight line between the triple point and wedge apex (schlieren photographs) or by drawing a straight line through regions of larger and smaller cell sizes (smoke foils). He compared the results with Whithams ray shock and three-shock theories by assuming the detonations were shock waves (unreactive case) or thin discontinuities with a heat release (reactive case). He found that smoke foils results compared well with those of Meltzer for hydrogen-oxygen but poorly with his shadowgraph measurements. None of the mixture tested agreed with the reactive shock solution, which Akbar claimed it is due to the fact that the cellular effects of the detonation are ignored in the theoretical calculations. He found that the experimental results for the larger wedges $\left(30^{\circ}+\right)$ had a tendency towards the reactive Whitham solution. The diluted mixture of hydrogen-oxygen was the exception to that rule as it had good agreement with the unreactive three shock theory within $2^{\circ}$. Akbar mentions that within the experimental field of view the phenomenon appeared to be self-similar for two mixtures. He also reports a critical angle greater than $45^{\circ}$ for both diluted and undiluted hydrogen-oxygen at $20 \mathrm{kPa}$ and between $40^{\circ}-45^{\circ}$ for diluted acetylene-oxygen at 50kPa. Although Akbar [1] said that the experiments appeared self-similar within his field of view it is not so. In the case of shockwaves no length scale other than the distance traveled is present, which lead to the assumption of self-similarity. The self-similar behavior of Mach reflection for shock waves 
was experimentally demonstrated by Bleakney et al. [4], [3] and Bazhenova [2]. However Hornung [16], [15] pointed out that this assumption would be wrong if the problem had such an independent length scale. Detonation waves have the length scale associated with them based on the interaction of chemical reactions with gas-dynamics in the form of the cell size $\lambda$. In order to compare his mixtures with one another Akbar took only pictures in the far-field region using the dimensionless characteristic length $\mathrm{L} / \lambda$, where $\mathrm{L}$ is the horizontal distance traveled by the incident wave, as a measure of self-similarity regions. This was done following a study by Sandeman et al. [27] who studied the diffraction of strong shockwaves along wedges. Strong shockwaves have a thickness associated with them in the form of a relaxation length, which is a length behind the shockwave at which chemical equilibrium is established and is very similar to the detonation cell size $\lambda$. Akbar defines the two extreme cases as:

- Small L/ $\lambda$ : corresponding to the frozen chemistry (unreactive shockwaves) in which the experiments are expected to favorably compare with the unreactive theories. On the wedge this region is represented by the near field very close to the apex.

- Large $\mathrm{L} / \lambda$ : corresponding to the equilibrium chemistry (thin discontinuity detonation wave) in which experiments are expected to compare favorably with the reactive theories. On the wedge this region is considered to be the far-field.

Akbar focused only in the far-field region in which L is large and self-similarity is expected, thus the conclusion that the phenomenon appeared self-similar is not representative of the actual phenomenon.

Shepherd et al. (1999) [30] reviewed the results obtained by Akbar and Meltzer. He provided a good discussion on the failure of self-similarity due to the detonation wave 
thickness. He points out that smoke foils are not an effective way to measure the triple point in mixtures with irregular cellular structure, like hydrogen-oxygen, because a change in cell size may not be an indication of the triple point location. From smoke foils it is difficult to distinguish the triple point from the Mach reflection to that of an instability wave triple point inherent to irregular structure detonation wave. He also pointed out that the triple point is not sharply defined as in shockwaves and it has a tendency to be smoothly distributed over a range (especially for the wedges below $20^{\circ}$ ) and this is a major source of error in experimental results. The experimental results are found to be "bounded" by the unreactive and reactive versions of the three-shock and Ray shock theories. This led him to believe that mixtures with different reaction zone length would have different triple point trajectories as well as different critical transition angle.

Hoshi (1999) [17] studied Mach reflection of detonations in stoichiometric hydrogenoxygen with various degree of argon dilution at various initial pressures ranging from 29.3 $\mathrm{kPa}$ to $53.3 \mathrm{kPa}$ over $10^{\circ}, 20^{\circ}, 30^{\circ}, 35^{\circ}, 40^{\circ}$ wedges. They used schlieren photographs and smoke foils to observe the phenomenon. They measured the maximum pressure behind the Mach stem and compared it with the 2-gamma three-shock theory for thin discontinuity detonation developed by Li et al. (1997) [20] for the Mach reflection of detonation. The theoretical results underpredicted the experimental results. It was also found that the triple point trajectory is not straight and that there was an induction distance prior to the establishment of the Mach stem detonation.

Ohyagi et al. (2000) [25] performed numerical experiments with diluted stoichiometric hydrogen-oxygen at 1atm over wedges carrying between 0 and 40 degrees. They found that the triple point trajectory, $\chi$, is not a straight line. They also found that the cell shape 
behind the Mach stem are determined from the interactions between transverse waves and the wedge surface. Although they did not obtain a well resolved structure behind the Mach stem in the calculations, they could observe a significant structure difference between the incident wave and Mach stem.

Guo et al. (2001) [13] used smoke foils to study Mach reflection for detonations in hydrogen-oxygen mixtures diluted with $25 \%$ argon in two sets of experiments. The first series of tests were made by changing the wedge angle from $10^{\circ}$ to $45^{\circ}$ and keeping the initial pressure constant at $16 \mathrm{kPa}$. The second set of experiments were performed on a constant $19.3^{\circ}$ wedge and varying the initial pressure from $16 \mathrm{kPa}$ to $40 \mathrm{kPa}$. From the first set of experiments it was concluded that the critical angle for transition was between $50^{\circ}$ $53^{\circ}$. This angle was found using an exponential fit of the experimentally measured triple point trajectory $\chi$. It was found that $\chi$ does not move in a straight line. The explanation presented was that the transverse wave interactions changed the trajectory. Since $\chi$ does not move in a straight line the experimentally measured $\chi$ was done by measuring the angle made by a linear fit to the irregular line fitted to the wedge apex. Also it was mentioned that for wedges $30^{\circ}$ and higher the triple point seemed to move straight. However the channel height used in this study was $4 \mathrm{~cm}$ which means that the wedges were short. This means that not enough time is given for the Mach stem to develop. The second set of experiment concluded that the initial pressure had no effect on $\chi$, but only the results up to $27 \mathrm{kPa}$ are shown.

Thomas and al. (2002) [31] studied detonation of stoichiometric hydrogen-oxygen, ethane-oxygen, acetyelene-oxyegn and acetyelene-nitrous oxide diluted with argon and nitrogen over wedges, as well as curved channel and curved pipes. They investigated the 
effects of the cellular structure on the reflection process using smoke foils and schlieren photographs. They concluded that a structure variation for detonation waves with a relatively regular structure had minimal impacts on the diffraction process. However mixtures with irregular structures did not yield conclusive results because the internal structure variation due to microscopic shock interactions masked the macroscopic Mach reflection process. They claim that the presence of transverse waves has an influence on the reflection process and that it is probably not self-similar.

Wang et al. (2009) [33] did a numerical study of the Mach reflection of detonations in hydrogen-oxygen diluted with $25 \%$ argon at $8 \mathrm{kPa}$ initial pressure. They simulated wedges ranging from $5^{\circ}$ to $50^{\circ}$ and compared their results with those of Guo (2001) [13]. Due to the cellular structure a detonation front is not planar, however when the cell size of the mixture is small the non-planar effects can be ignored but when detonations have a low initial pressure the cells are large and becomes comparable to the size of the Mach stem (or the channel width) the non-planar front cannot be disregarded. In this case it was found that for all wedges the process started with regular reflection which then transited to Mach reflection further up the wedge. The distance required for this transition increased with wedge angle. Although the transition angle was found to be the same as Guo, the simulated results overpredicted the experimental data. Based on the conclusion from Guo, that initial pressure has no effect on the trajectory of $\chi$, they eliminated the possibility that the different initial pressure was the cause of the discrepancy. This is erroneous because Guo concluded that initial pressure had no impact on the trajectory however he started at $16 \mathrm{kPa}$ and did not mention the effects of lower initial pressure. The difference in the 
detonation front and cellular structure between $16 \mathrm{kPa}$ and $8 \mathrm{kPa}$ is very large and should not be dismissed so easily.

\subsection{Three-Shock Theory Limitations}

As was mentioned previously, the wedge angle is the most influencial parameter in Mach reflection. When the wedge angle is large, regular reflection is obtained and thus the triple point trajectory $\chi$ is $0^{\circ}$. However when the wedge angle is sufficiently small Mach reflection occurs and the trajectory angle $\chi$ can be calculated. The relationship between $\chi$ and the wedge angle $\left(\theta_{w}\right)$ is shown in Fig. $1-8$ for a mixture of $\mathrm{C}_{2} \mathrm{H}_{2}+2.5 \mathrm{O}_{2}$ $+70 \% \mathrm{Ar}$ at $20 \mathrm{kPa}$. The unreactive case represents the frozen chemistry case (shockwave travelling at $\mathbf{M}_{c j}$ assumption) and the reactive case represents the equilibrium chemistry case (thin discontinuity assumption). In both cases the complex three-dimensional effects of the transverse waves are not considered and thus it represents a gross simplification of a real cellular detonation wave. The complex structure of a cellular detonation wave greatly influences the flowfield behind the wave and these effects should not be ignored. In the past, the trajectory of the triple point was also compared with results from the Whithams Ray shock theory. However since this theory requires the presence of a Mach stem to solve for $\chi$, it cannot be used to find the critical angle of transition. This method was also deemed inappropriate to use in the reactive case by Shepherd and will not be used further in this study. 


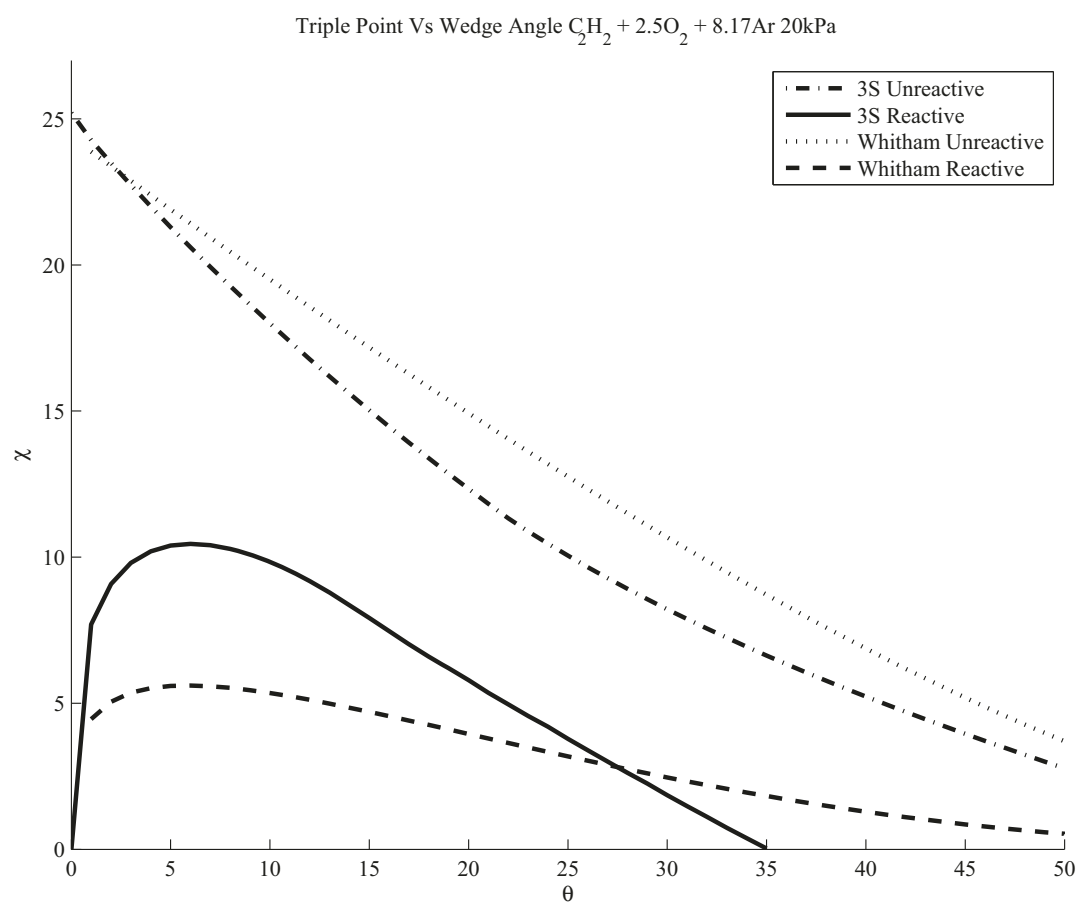

Figure 1-8: Relationship between Triple point trajectory and wedge angle

Using the thin discontinuity model makes the change of initial pressure have minimal influence on the trajectory of the triple point. This is because the heat release parameter $\mathrm{Q}$ is a function of the detonation wave Mach number (as discussed in Appendix B). When the initial pressure is lowered, so is the Mach number, thus is Q. These changes counteract each other and the calculated results remain the same. This was demonstrated by Gavrilenko [10] who found that the mixture and initial pressure used in three-shock theory had minimal impact on the critical angle for transition obtaining a value of $34 \pm 0.4^{\circ}$ for all undiluted mixtures for all initial pressures calculated. Dilution with argon has a significant effect on the cellular structure of the detonation wave, yet the trajectories of the triple 
point calculated with three-shock theory are marginally different from the undiluted case because the cellular structure is ignored, as shown in Fig. 1-9

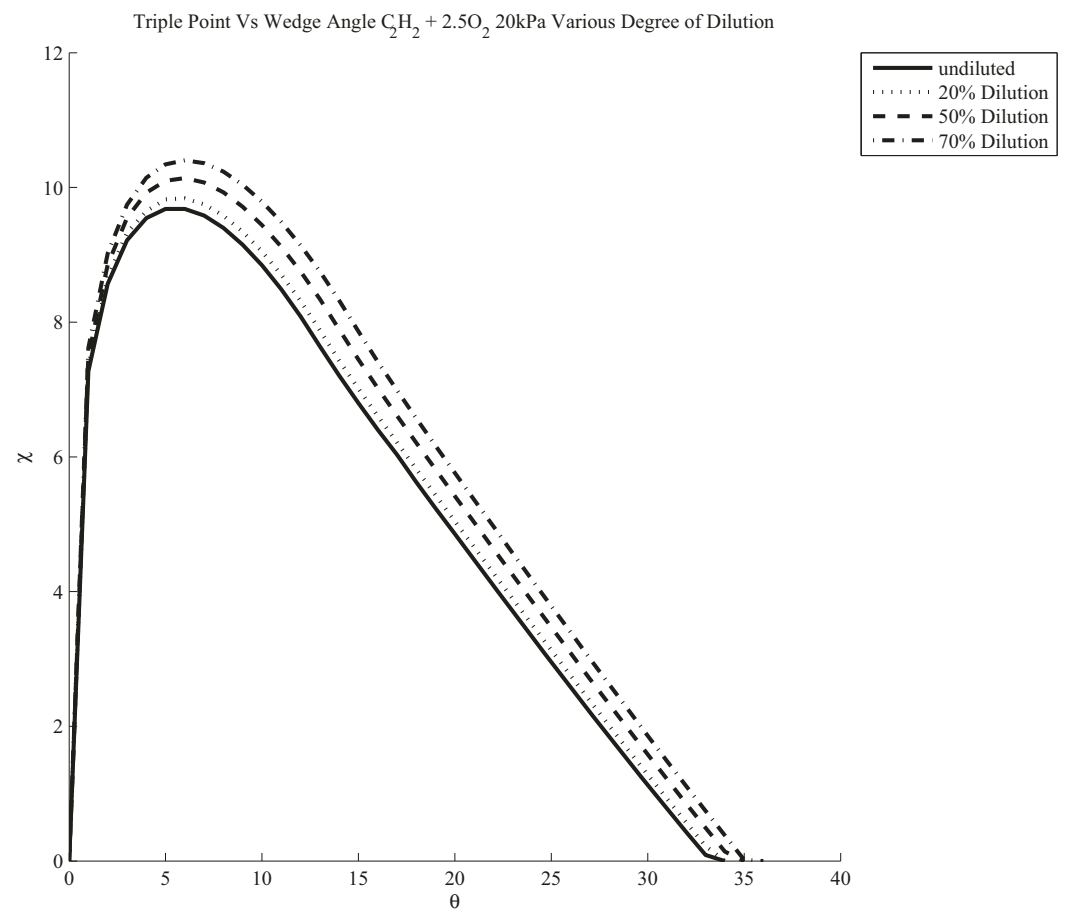

Figure 1-9: Reactive Three-shock theory for various dilution ratios of $\mathrm{C}_{2} \mathrm{H}_{2}+2.5 \mathrm{O}_{2}$ mixture

This is also in agreement with Meltzer [21] who calculated the theoretical triple point trajectory $\chi$ in a mixture of $\mathrm{H}_{2}+\mathrm{O}_{2}$ with various degree of dilution with argon and obtained similar results.

\subsection{Motivation}

Failure of self-similarity makes it means that the trajectory of the triple point is no longer a straight line starting from the apex of the wedge. Although a number of previous 
researchers have reported that the trajectory of the triple point is a curved line, they ignored the curvature and determine the angle $\chi$ between the triple point trajectory and the wedge surface to compare with the three-shock theory. In some cases where short wedges (or a single schlieren photograph) were used, a straight line was drawn from the apex of the wedge to the triple point. Thus the curvature is overlooked which is not representative of the phenomenon. In the experiments where smoke foils were used to record the Mach reflection process [13], the complete boundary between the Mach stem and incident detonation can be obtained. Previous investigators have reported a "wavy" boundary, however, a linear fit was used to obtain a straight line trajectory from the wedge apex. Some investigators also noted that the Mach stem does not necessarily starts from the wedge tip, but some distance downstream of the apex. According to Wang [33], this indicates that a relaxation region is required for the formation of the Mach stem. Yet he still determined a straight trajectory from the wedge apex to separate the difference in the cellular pattern observed between the Mach stem and the incident detonation. The effects of changing the initial pressure are also not well known. Guo mentioned that the initial pressure has no effect on the triple point trajectory, which is in contradiction with Shepherd that mentions that mixtures with different reaction zone lengths (or cell size) would behave differently. Furthermore Ong was the only researcher obtaining a good agreement between experimental and theoretical results. He performed his experiment at high pressures (20psia) and thus had small enough cell sizes compared with the channel width (or in the case of Mach reflection, the Mach stem) to allow for the thin discontinuity assumption to be valid. It is clear then that there is some confusion stemming from the past results and that the 
current understanding of the Mach reflection of a cellular detonation wave is not satisfactory in spite of the numerous investigations. It is therefore worthwhile to carry out a more extensive study to highlight the complex phenomenon of Mach reflection of cellular detonations. In the present study, we chose detonable mixtures with both regular and irregular cell pattern. We also vary the initial pressure to obtain a range of cell sizes of the incident detonation to change the characteristic length scale of the detonation front. Using schlieren photographs and smoke foils, we also determine the variation of the height of the Mach stem with incident wave travel distance. The smoked foils reveal the interaction of the transverse waves of the Mach stem with the incident detonation front at the junctions of the two waves. 


\section{CHAPTER 2 \\ Experimental Details}

\subsection{Experimental setup}

The experiments have been carried out in a rectangular aluminum tube, $1.14 \mathrm{~m}$ long by $0.10 \mathrm{~m}$ wide and $0.025 \mathrm{~m}$ thick supporting two opposing windows walls made of tempered glass to permit schlieren photography. A sketch of the configuration of the windowed section can be seen in Fig. 2-1. It was mounted vertically in the schlieren field of view.

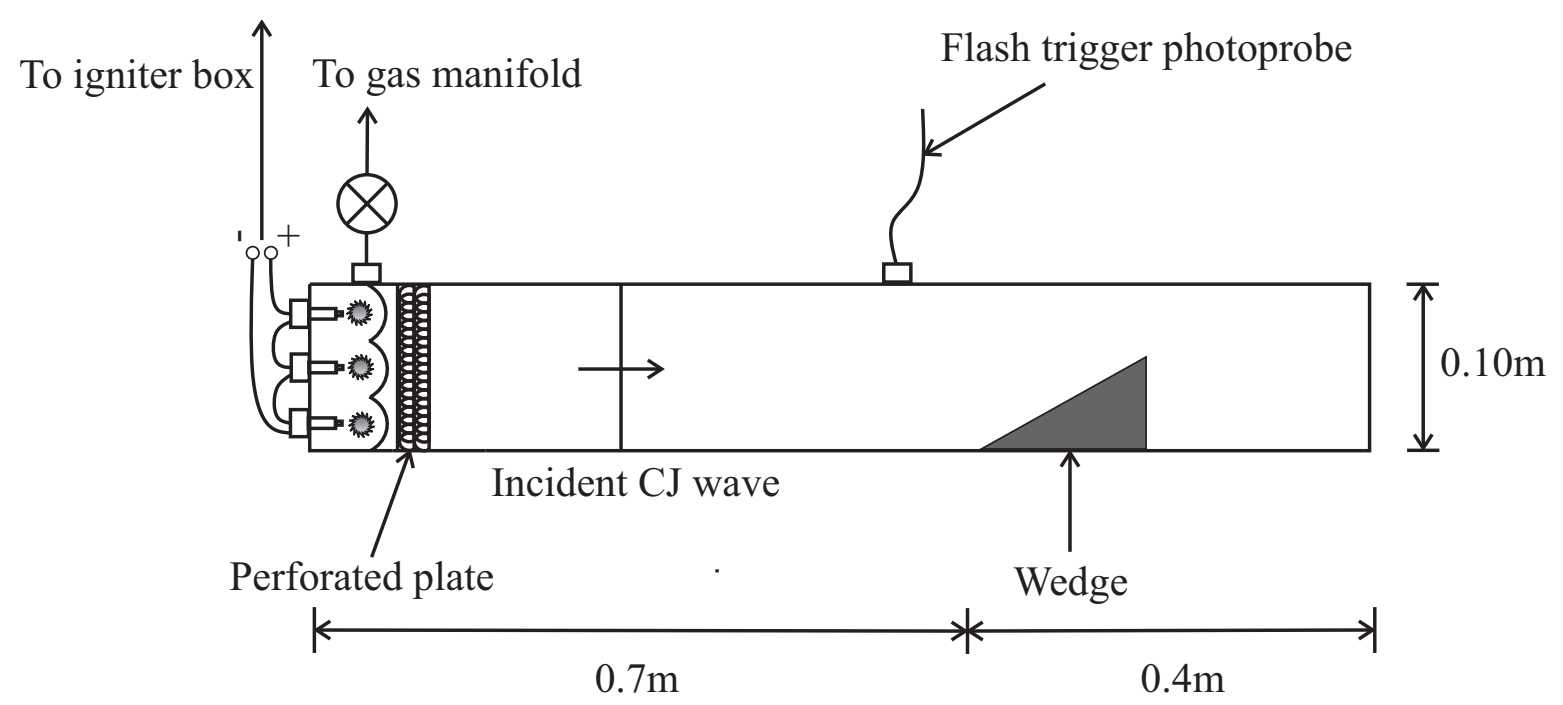

Figure 2-1: Schematic of the windowed section

Ignition of the detonation at top end of the tube was performed by means of 3 simultaneous powerful sparks obtained via the rapid discharge of a high-voltage capacitor across a gap switch. When less sensitive mixtures were used, a tiny amount of driver mixture was injected near the igniters a few seconds before initiation. Perforated plates 
holding coarse wire mesh near the igniters promoted instabilities in order to facilitate the formation of a CJ detonation in the vicinity of the igniters. The wedges were installed sufficiently far downstream as to have a fully developed CJ detonation. Five wedges have been used throughout the present study. They are $10^{\circ}, 20^{\circ}, 30^{\circ}, 35^{\circ}$ and $40^{\circ}$.

\subsection{Mixture Selection and Preparation}

Three mixtures were selected for use in the present study. They are: stoichiometric acetylene-oxygen undiluted $\left(\mathrm{C}_{2} \mathrm{H}_{2}+2.5 \mathrm{O}_{2}\right)$ and with $70 \%$ of argon dilution $\left(\mathrm{C}_{2} \mathrm{H}_{2}+\right.$ $\left.2.5 \mathrm{O}_{2}+70 \% \mathrm{Ar}\right)$ and stoichiometric propane-oxygen $\left(\mathrm{C}_{3} \mathrm{H}_{8}+5 \mathrm{O}_{2}\right)$. argon dilution gives a regular the cellular pattern as well as larger cell size as was discussed by Shepherd [18], [29], Radulescu [22] and Voitsekhovskii [5]. The initial pressure range used in the experiments was varied between $3 \mathrm{kPa}$ and $20 \mathrm{kPa}$. These three mixtures provide different cellular structures. Mixtures with different cellular structures have been found to behave differently under perturbations (Moen [24], Shepherd [18] and Desbores [6]). The mixtures

have been prepared using the method of partial pressure and given at least 18 hours to mix. The aforementioned driver mixture is equimolar acetylene-oxygen $\left(\mathrm{C}_{2} \mathrm{H}_{2}+\mathrm{O}_{2}\right)$ and is prepared in the same way.

\subsection{Diagnostics}

The main diagnostics used in this study were single-frame schlieren photography and smoke foils. The schlieren is setup in a z-type configuration. The single frame is obtained by a short duration spark flash. The flash duration is measured to be around 500ns. In order to properly coordinate the flash with the detonation wave in the field of view of a DSLR type camera, a digital delay generator is configured before each experiment. It is clear then that each photograph represents a different experiment; however for every time 
step, at least two experiments were performed to ensure that enough reproducibility in the pictures was found. This allowed for proper chronological reconstruction. The pictures are then digitized using MATLAB in order to measure the triple point height. See appendix A for more details about the schlieren setup and flash trigger.

However schlieren photographs are not a very good way to observe the effect of the different mixtures. Because the photographs integrate the density gradients throughout the entire width of the channel, the detonation front can appear much thicker when the cellular structure is irregular because the transverse waves are unevenly spaced. As such smoke foils are a much better approach to observe the interaction of the transverse waves. Smoke foils can be used to describe the changes in the detonation wave structure during the Mach reflection process. Since the Mach stem is overdriven it will have smaller cell sizes when compared with the incident wave. Using smoke foils, it is possible to investigate the Mach reflection process of cellular detonation by inspecting the foil to identify a region in which the cell size, closer to the wedge surface, are smaller than that of the incident wave. Smoke foils complement the schlieren photographs quite well to give a global view of the phenomenon. Schlieren are instantaneous view of the wave configurations at any given time. Smoke foils however give the cellular structure behind the incident wave and Mach stem throughout the whole propagation over the wedge. Although smoke foils are usually limited to lower pressures for clarity (the cell sizes becoming very small at high pressure thus rendering the cell size difference between the incident wave and the Mach stem much harder to differentiate) unlike schlieren photographs. Each method complements the other. 


\section{CHAPTER 3 \\ Results and Discussion}

\subsection{Effect of Initial Pressure}

To investigate the effect of cell size on the Mach reflection phenomenon, the initial pressure is varied. Fig. 3-1 shows the Mach reflection of a detonation wave in $\mathrm{C}_{2} \mathrm{H}_{2}+$ $2.5 \mathrm{O}_{2}+70 \% \mathrm{Ar}$ at an initial pressure of $20 \mathrm{kPa}$ on a $30^{\circ}$ wedge. The detonation cell size under this condition corresponds to about $2 \mathrm{~mm}$. Near the end of the wedge the Mach stem height, measured from the perceived triple point and the wedge surface, is about $10 \mathrm{~mm}$ and is greater than the cell size. A distinct difference in the cell structure between the Mach stem and the incident detonation can be observed and the change in structure gives a more definite location of the triple point. 


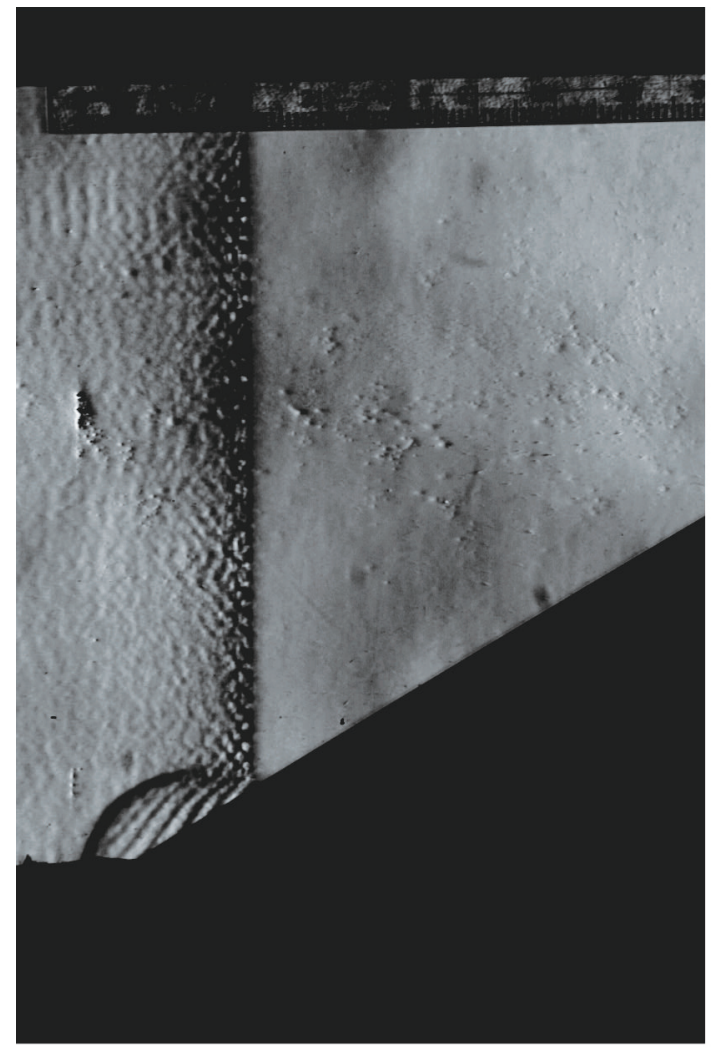

a)

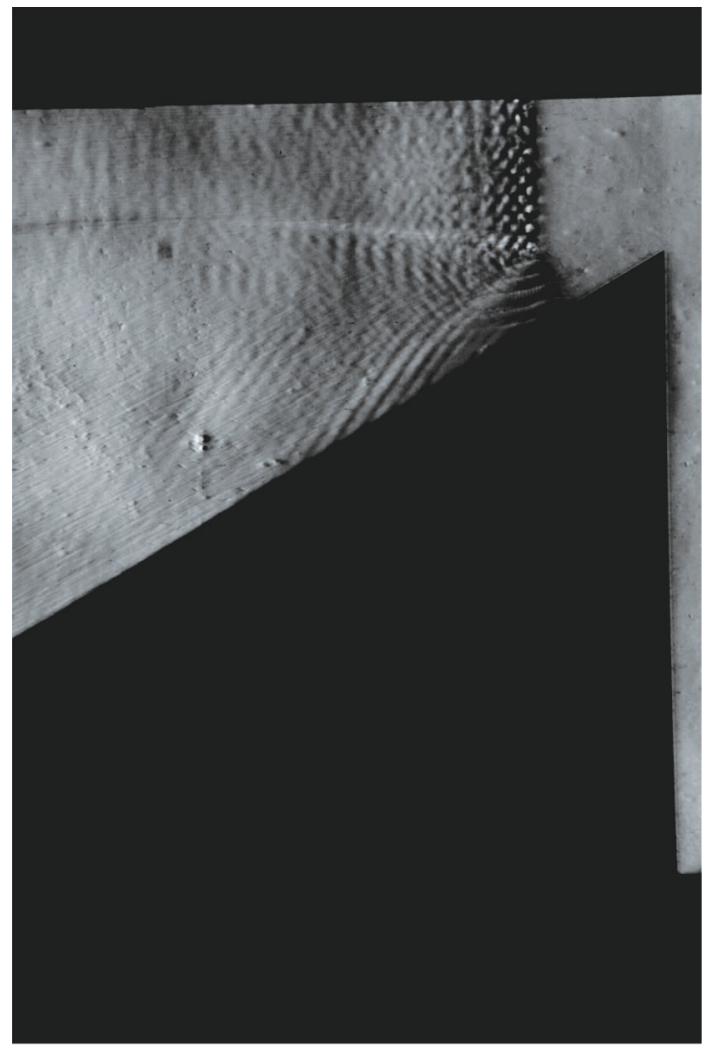

b)

Figure 3-1: Detonation wave in $\mathrm{C}_{2} \mathrm{H}_{2}+2.5 \mathrm{O}_{2}+70 \%$ Ar at $20 \mathrm{kPa}$ over $30^{\circ}$ wedge a) near-field b) far-field

Reducing the initial pressure down to $3 \mathrm{kPa}$ in Fig. 3-2 gives the mixture a cell size of about $37.7 \mathrm{~mm}$. The reflected wave is not evident in this case however the triple point region can still be localized due to the distinct difference in the structure between the Mach stem and the incident wave. A pronounced "thinning" of the thickness of the detonation of the Mach stem when compared with the incident wave can be observed. The Mach stem height is roughly $20 \mathrm{~mm}$ which is significantly larger than the $20 \mathrm{kPa}$ case. This illustrates 
a case in which the Mach reflection of a cellular detonation where the cell size is large and the detonation cannot be approximated as a thin discontinuity even at the end of the wedge

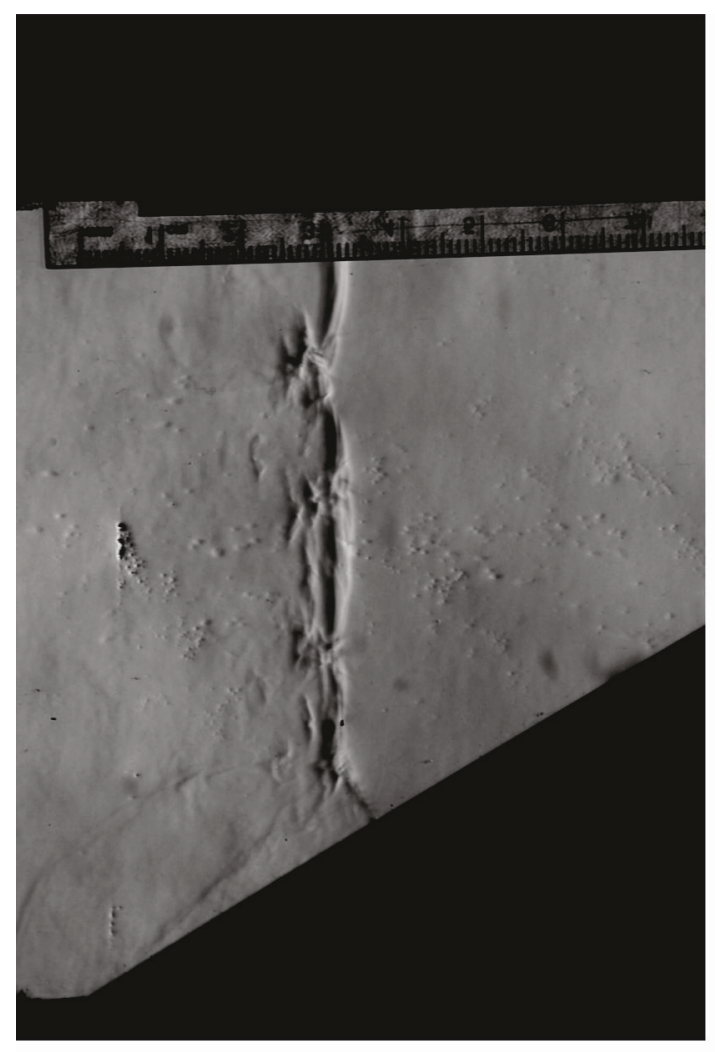

a)

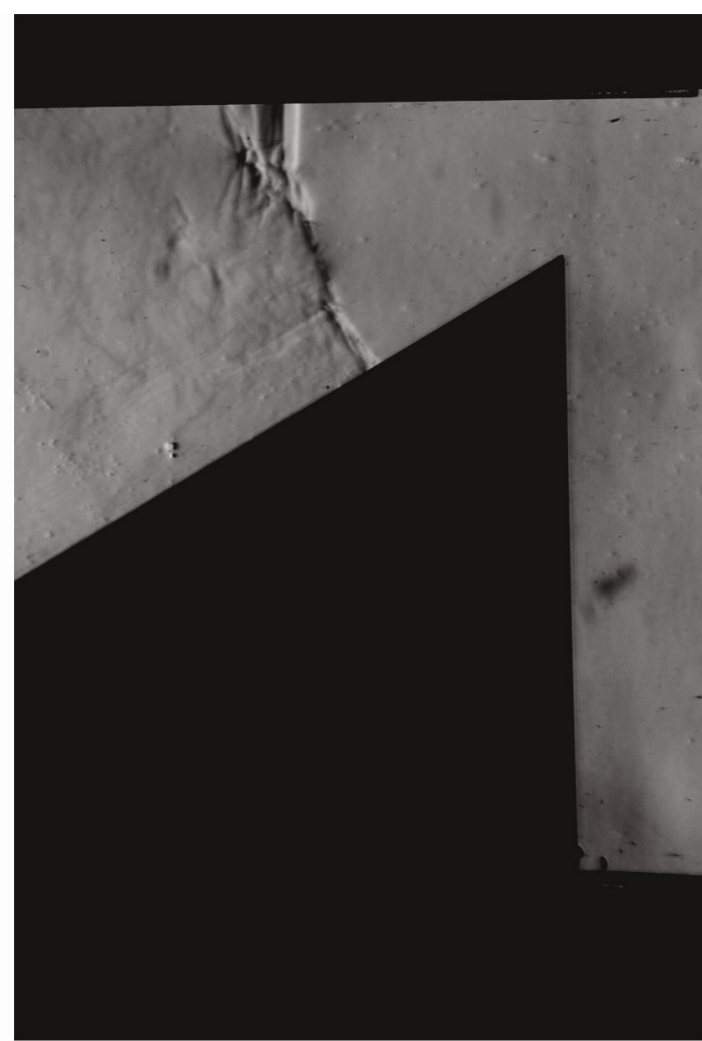

b)

Figure 3-2: Detonation wave in $\mathrm{C}_{2} \mathrm{H}_{2}+2.5 \mathrm{O}_{2}+70 \%$ Ar at $3 \mathrm{kPa}$ over $30^{\circ}$ wedge a) near-field b) far-field

\subsection{Effect of Wedge Angle}

Fig. 3-3 shows the Mach reflection of a detonation wave in $\mathrm{C}_{2} \mathrm{H}_{2}+2.5 \mathrm{O}_{2}+70 \% \mathrm{Ar}$ at $20 \mathrm{kPa}$ over a smaller angle of $\theta_{w}=10^{\circ}$. The reflected wave is hardly seen in the nearfield. There is evidently no distinct triple point discernable in this case and the Mach stem is smoothly curved to join the incident wave. The reflected wave is also hard to discern 
even in the far-field and the cellular structure along the curved portion of the wave appears to remain more or less the same as that of the incident wave.

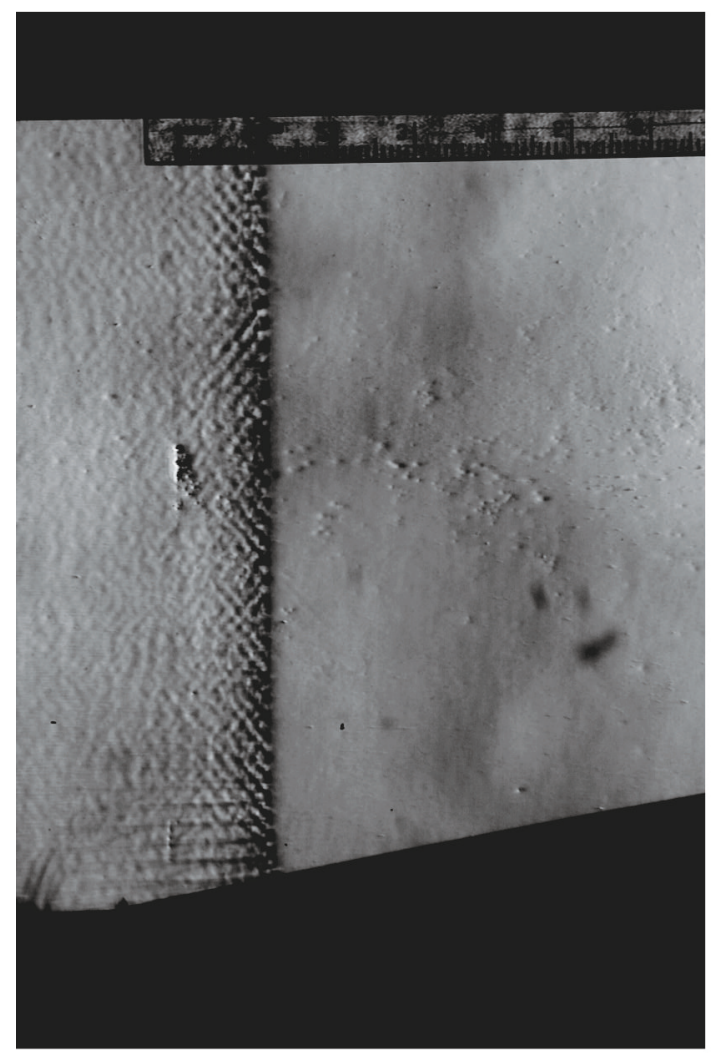

a)

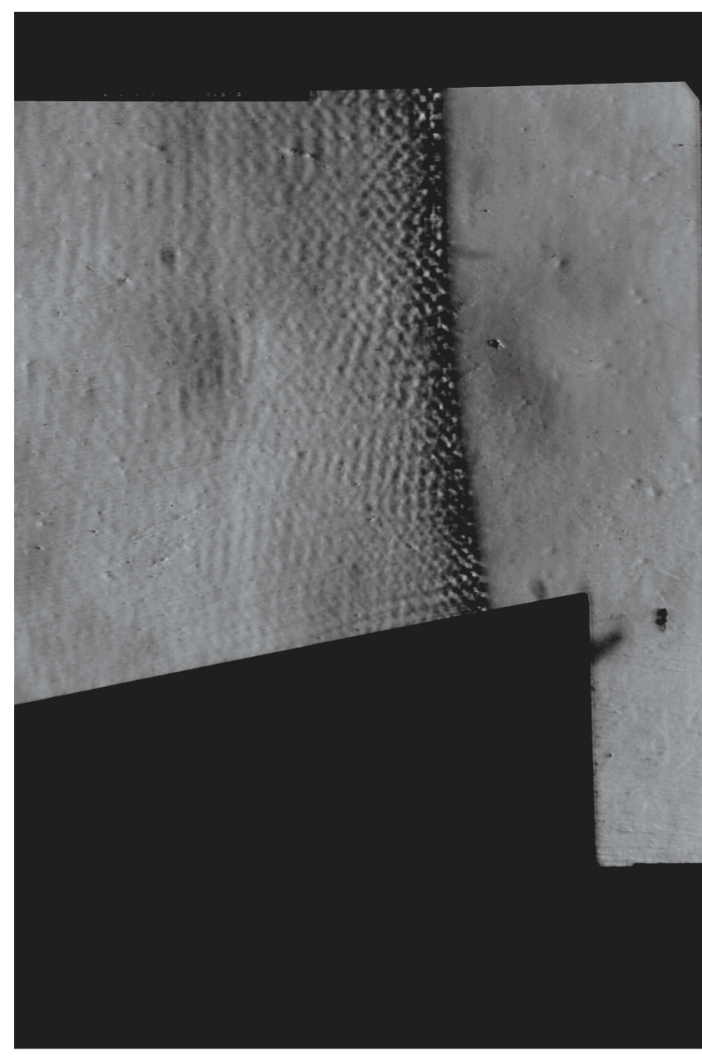

b)

Figure 3-3: Detonation wave in $\mathrm{C}_{2} \mathrm{H}_{2}+2.5 \mathrm{O}_{2}+70 \% \mathrm{Ar}$ at $20 \mathrm{kPa}$ over $10^{\circ}$ wedge a) near-field b) far-field

When the wedge angle is increased to $\theta_{w}=35^{\circ}$ the reflected wave becomes stronger and thus it can be more clearly observed even in the far-field. as in Fig. 3-4 shows the transition between the incident wave and the Mach stem becomes much clearer with the appearance of a definite "kink" in the wave. The structure difference between the Mach stem and incident wave is also noticeable. The appearance of striations is also more 
prominent. These striations appear to be a train of compressive waves generated by the interaction between the Mach stem transverse waves and the wedge surface.

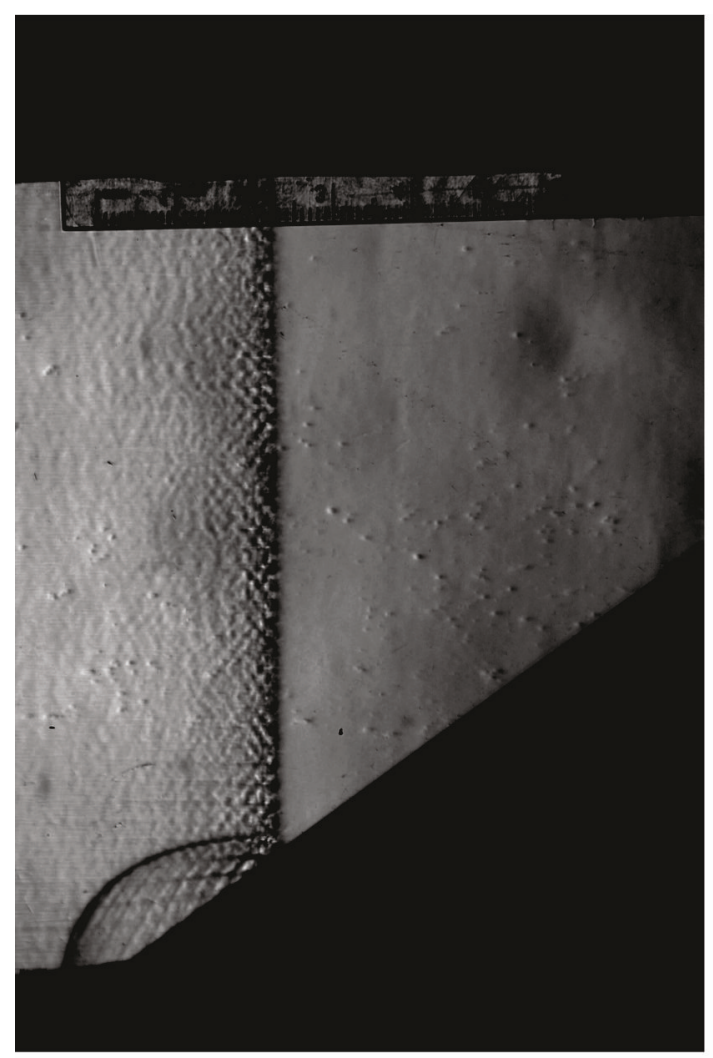

a)

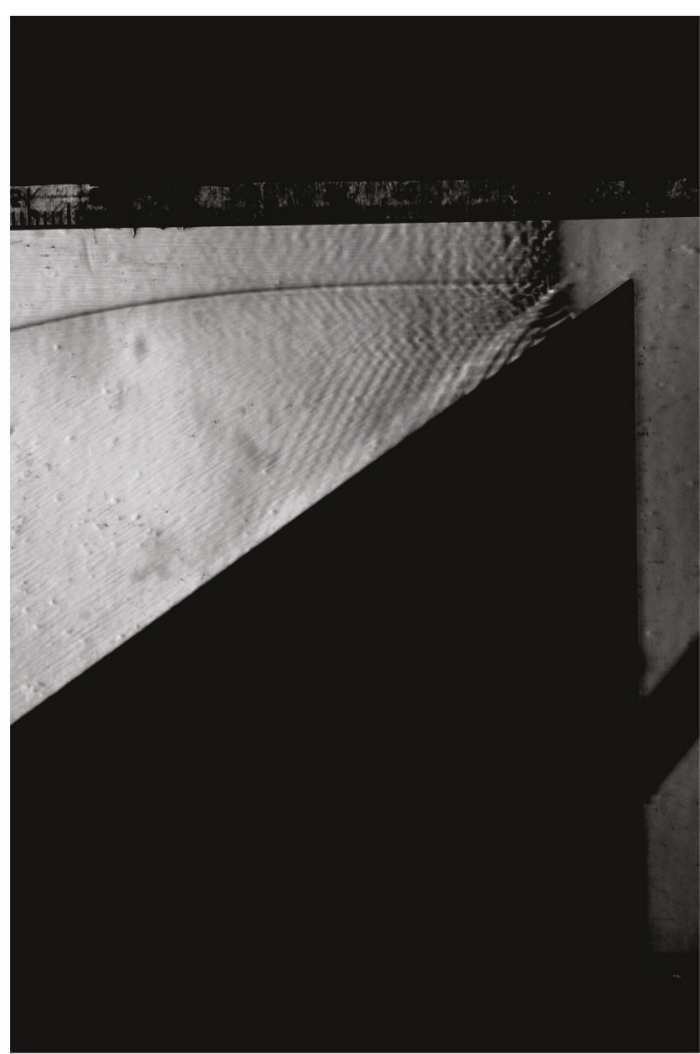

b)

Figure 3-4: Detonation wave in $\mathrm{C}_{2} \mathrm{H}_{2}+2.5 \mathrm{O}_{2}+70 \%$ Ar at $20 \mathrm{kPa}$ over $35^{\circ}$ wedge a) near-field b) far-field

It is expected that the Mach stem height would be shorter when the wedge angle becomes larger since the triple point trajectory decreases for larger wedge angles, as demonstrated in Fig. 1-8, Mach reflection of detonations over small wedge angles was demonstrated to not yield a Mach stem. When the wedge angle becomes very small (usually $\theta_{w}$ $<15^{\circ}$ ) the reflected wave becomes extremely weak. Shock waves depend on the flowfield 
behind them to dictate their propagation. Even a weak reflected shock would increase the pressure in the region below the triple point, thus driving the Mach stem forward. However since detonation waves propagate from the chemical energy release and the expansion of the products, any pressure gradient behind the sonic plane has no impact on the detonation front. In this case a weak reflected shock would "blend in" with the transverse waves of the detonation and the front would accommodate itself to the wedge surface. In contrast when the wedge angle is large the reflected wave is very strong and will affect the transverse waves from the incident detonation. The transverse waves moving downward through the strong reflected wave propagating upward would slow down thus the wave spacing between consequent waves would become smaller leading to an effective smaller cell size behind the Mach stem. Furthermore the transverse waves reflecting off the wedge surface would also be much stronger, affecting the reaction rate of the Mach stem detonation and overdriving it.

\subsection{Effect of Mixture}

A detonation wave in $\mathrm{C}_{3} \mathrm{H}_{8}+5 \mathrm{O}_{2}$ with initial pressure of $10 \mathrm{kPa}$ over a $10^{\circ}$ wedge is shown in Fig. 3-5. $\mathrm{C}_{3} \mathrm{H}_{8}+5 \mathrm{O}_{2}$ gives an irregular cell pattern in contrast with detonations in highly argon diluted mixtures in previous figures. However very similar results are obtained. It can be seen that similarly to the $\mathrm{C}_{2} \mathrm{H}_{2}+2.5 \mathrm{O}_{2}+70 \% \mathrm{Ar}$ mixture, the reflected shock is fairly weak and cannot be defined. The Mach stem curved continuously to join the incident detonation and the difference between the structure in the overdriven Mach stem and the incident detonation is also not distinguishable. In Fig. 3-6 where the wedge angle is $35^{\circ}$, the reflected shock is very clear and the Mach stem is much smaller. The reflected shock appears to attenuate where the Mach stem is and it is difficult to define near 
the end of the wedge (most likely due to the density gradients of the incident detonation masking the weaker reflected wave). The difference in the detonation structure between Mach stem and incident detonation is also clear. It can also be seen that the Mach stem curves smoothly towards the incident detonation and the triple point is not a sharp point but rather a region not easily defined. The train of compression waves is also observed behind the Mach stem as in the case for the $\mathrm{C}_{2} \mathrm{H}_{2}+2.5 \mathrm{O}_{2}+70 \%$ Ar mixture.

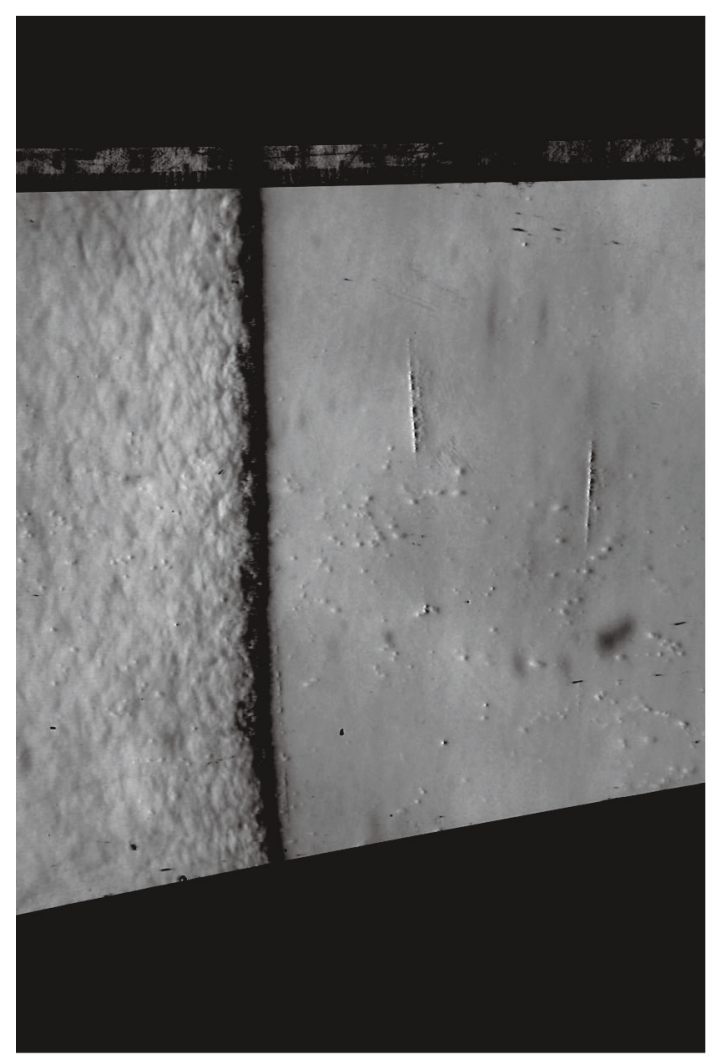

a)

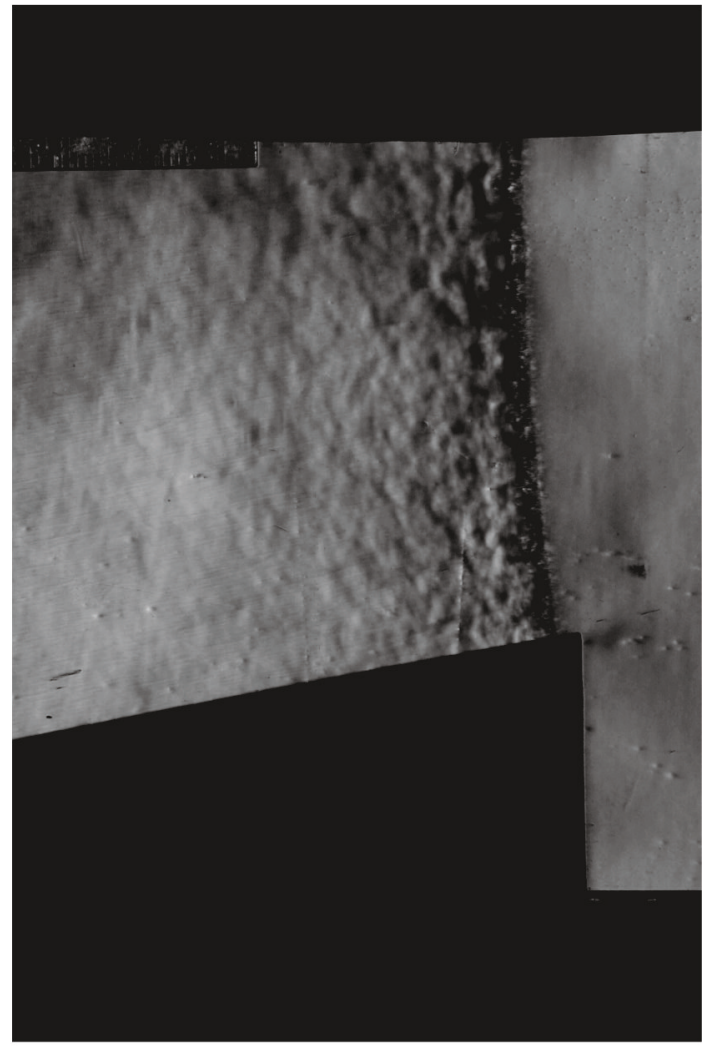

b)

Figure 3-5: Detonation in $\mathrm{C}_{3} \mathrm{H}_{8}+5 \mathrm{O}_{2}$ at $10 \mathrm{kPa}$ wave over $10^{\circ}$ wedge a) near-field b) far-field 


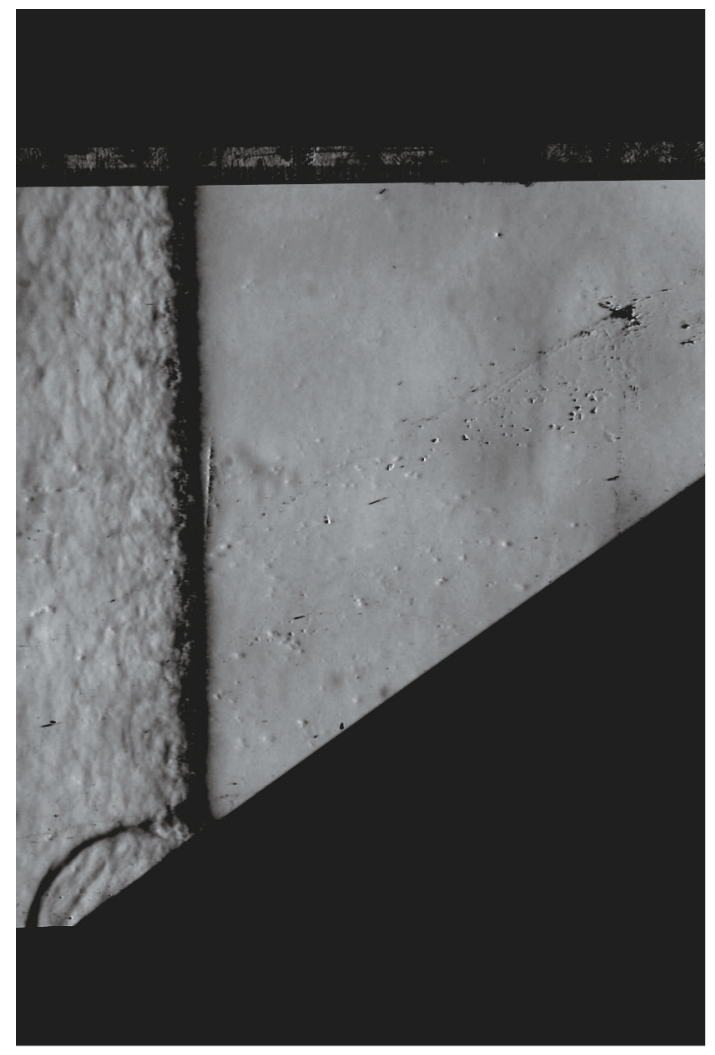

a)

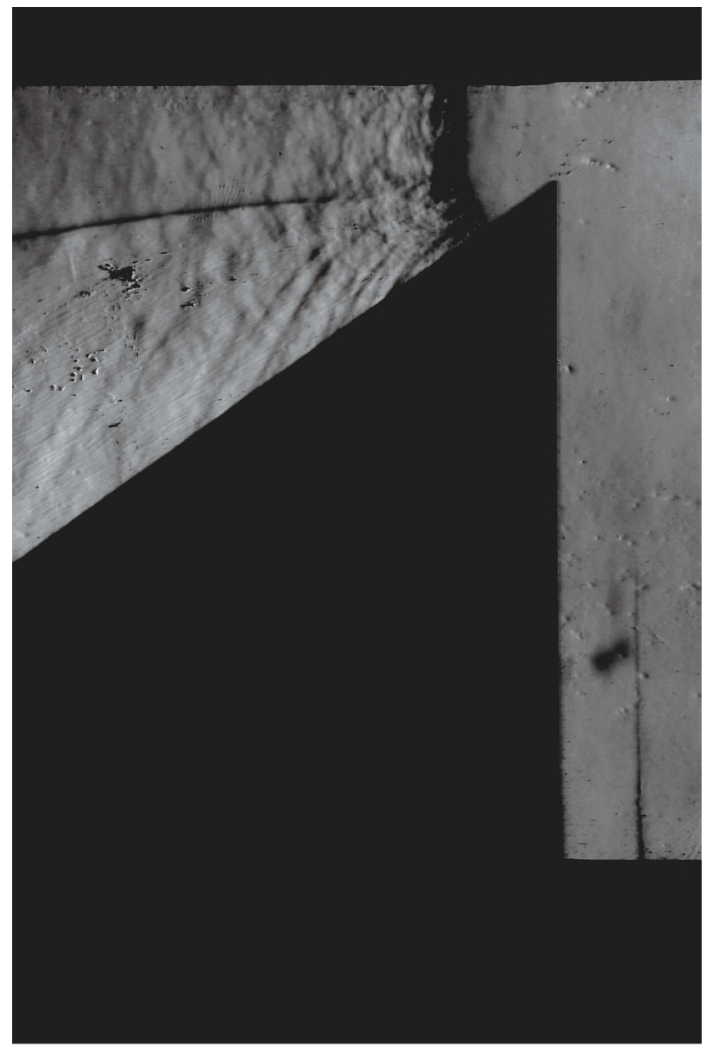

b)

Figure 3-6: Detonation wave in $\mathrm{C}_{3} \mathrm{H}_{8}+5 \mathrm{O}_{2}$ at $10 \mathrm{kPa}$ over $35^{\circ}$ wedge a) near-field b) far-field

The undiluted $\mathrm{C}_{2} \mathrm{H}_{2}+2.5 \mathrm{O}_{2}$ mixture presents some differences compared to the previous two mixtures as can be seen in Fig. 3-7, which shows a detonation in $\mathrm{C}_{2} \mathrm{H}_{2}+2.5 \mathrm{O}_{2}$ at $20 \mathrm{kPa}$ over a $10^{\circ}$ wedge. The overall detonation wave is much thinner for this mixture in comparison with the previous ones. This is due to the extremely rapid chemical reactions across the wave leading to very small cells (about $1 \mathrm{~mm}$ ) making detonations in this mixture appear similar to that of a shockwave with a heat release (thin discontinuity assumption). Unlike the previous results a reflected wave is visible throughout the entire 
propagation even for a $10^{\circ}$ wedge. A slight structural change between the Mach stem and incident detonation front observed as a "thinning" of the wave in the Mach stem. Although the other mixtures had no Mach stem for this wedge angle, one is observed in this mixture. However the transition between Mach stem and incident front is not sharp like in shockwaves. The Mach stem is heavily curved and the reflected wave appears almost tangent with the incident front at the contact location, thus blurring the exact location into a region. Fig. $3-7$

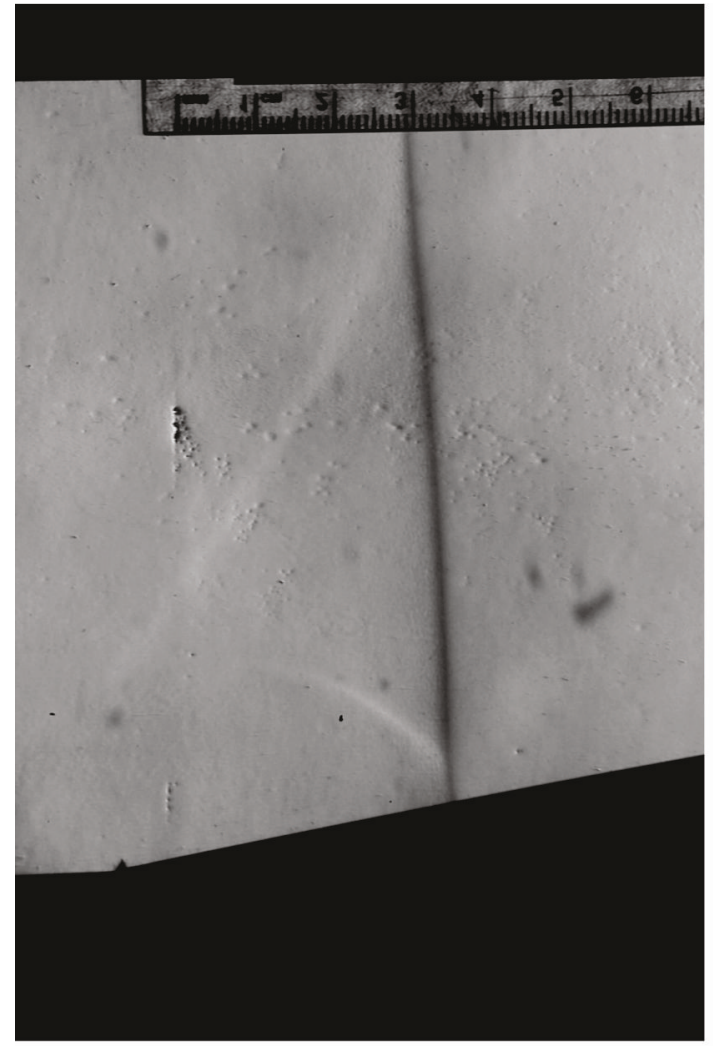

a)

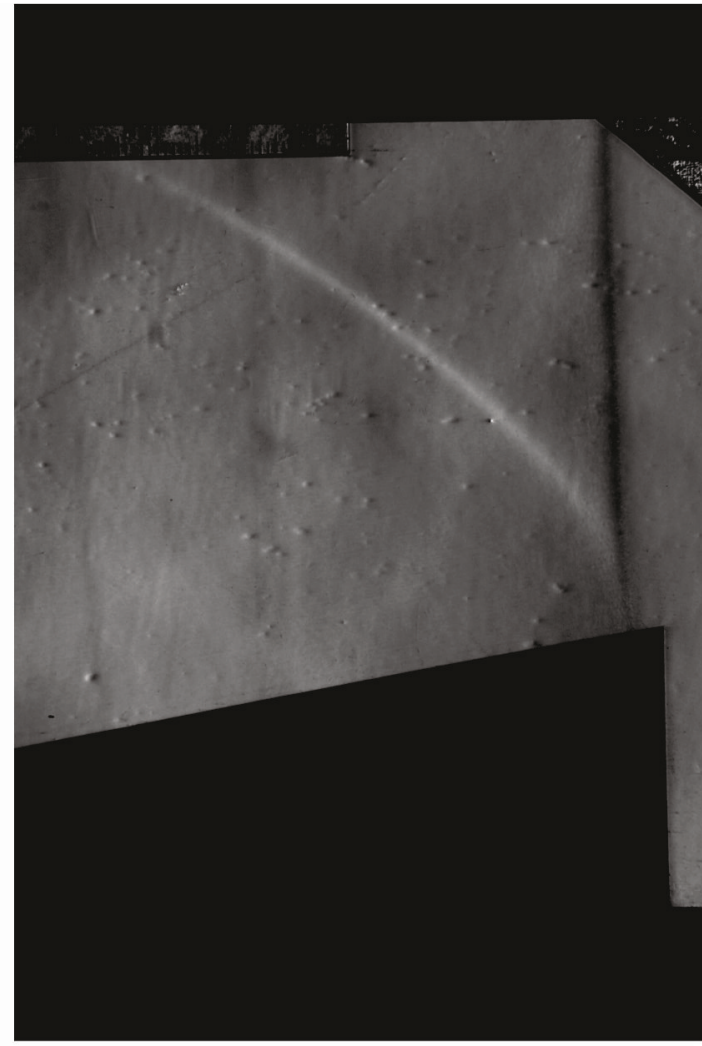

b)

Figure 3-7: Detonation wave in $\mathrm{C}_{2} \mathrm{H}_{2}+2.5 \mathrm{O}_{2}$ at $20 \mathrm{kPa}$ over $10^{\circ}$ wedge a) near-field b) far-field 
Increasing the wedge angle further, to $35^{\circ}$ in Fig. 3-8 shows regular reflection instead of Mach reflection. This is a major difference between this mixture and the previous ones. It is likely that the high sensitivity of this mixture allows for the Mach to develop much quicker thus reaching a steady state faster than the previous mixtures. This may explain why the Mach stem is smaller.

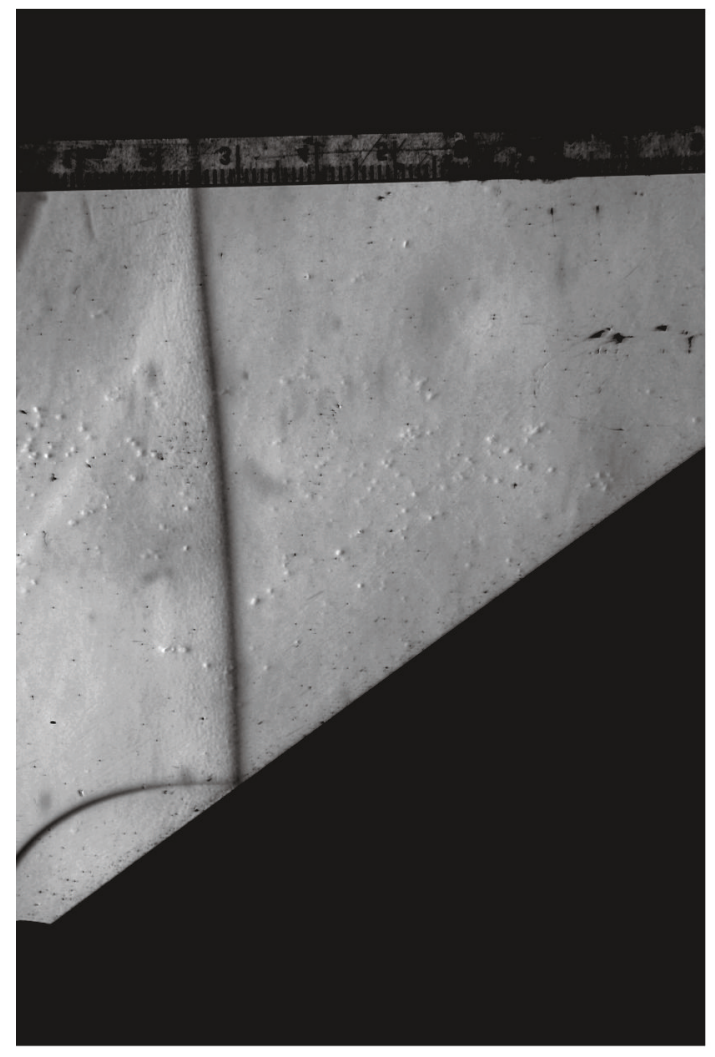

a)

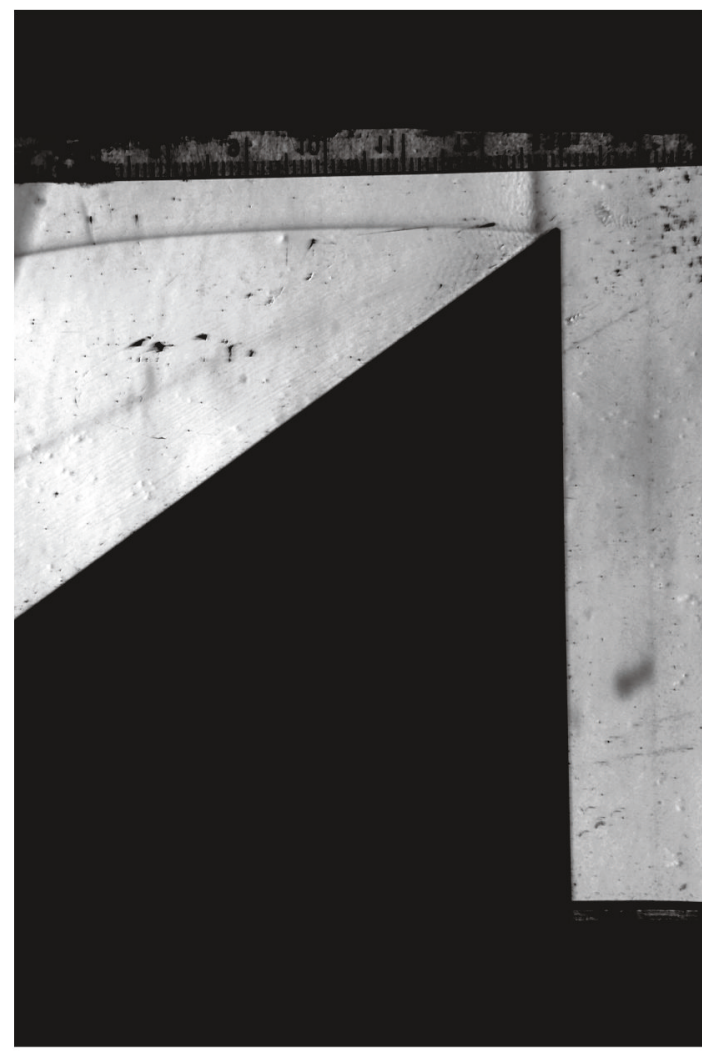

b)

Figure 3-8: Detonation wave in $\mathrm{C}_{2} \mathrm{H}_{2}+2.5 \mathrm{O}_{2}$ at $20 \mathrm{kPa}$ over $35^{\circ}$ wedge a) near-field b) far-field 


\subsection{Critical Angle for Transition}

According to the three-shock theory the critical angle for transition between regular reflection and Mach reflection is independent on the mixture (unless dilution with argon is present) and on the initial pressure. The calculated critical angle is about $34^{\circ}$. Based on the previous $\mathrm{C}_{2} \mathrm{H}_{2}+2.5 \mathrm{O}_{2}$ results at $20 \mathrm{kPa}$ it was shown that regular reflection was obtained for the $35^{\circ}$ shown in Fig. 3-10 but not at the $30^{\circ}$ wedge shown in Fig. 3-9. Thus the conclusion can be made that the critical angle for transition for this mixture is between $30^{\circ}-35^{\circ}$, which is in good agreement with the three-shock theory.

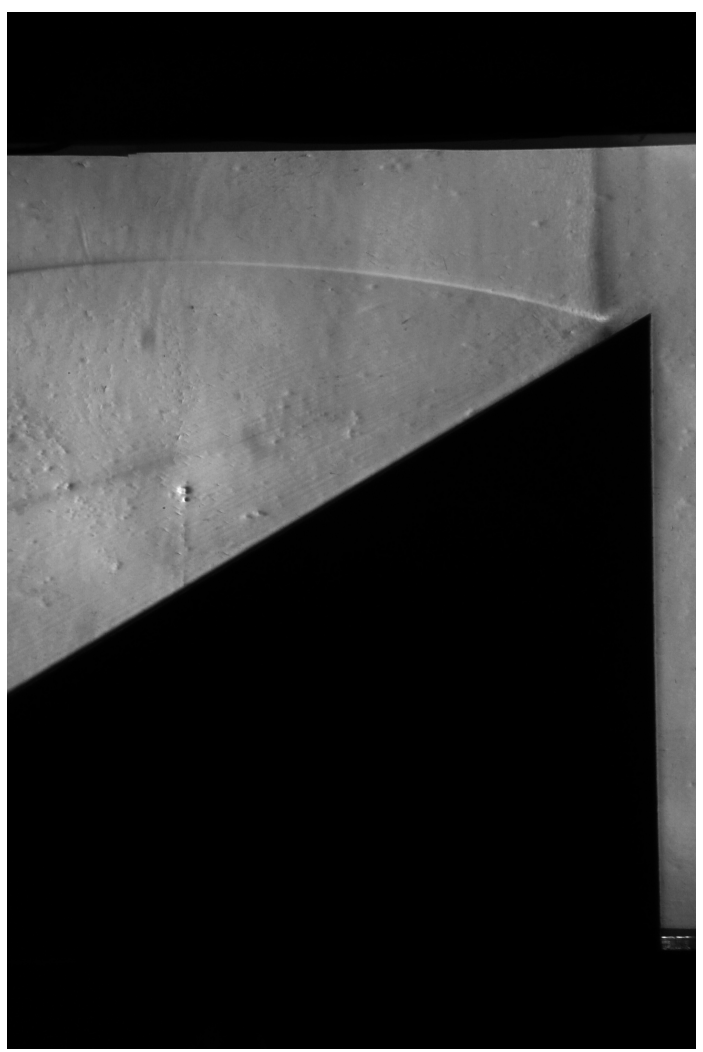

Figure 3-9: Detonation wave in $\mathrm{C}_{2} \mathrm{H}_{2}+2.5 \mathrm{O}_{2}$ at $20 \mathrm{kPa}$ over $30^{\circ}$ wedge 


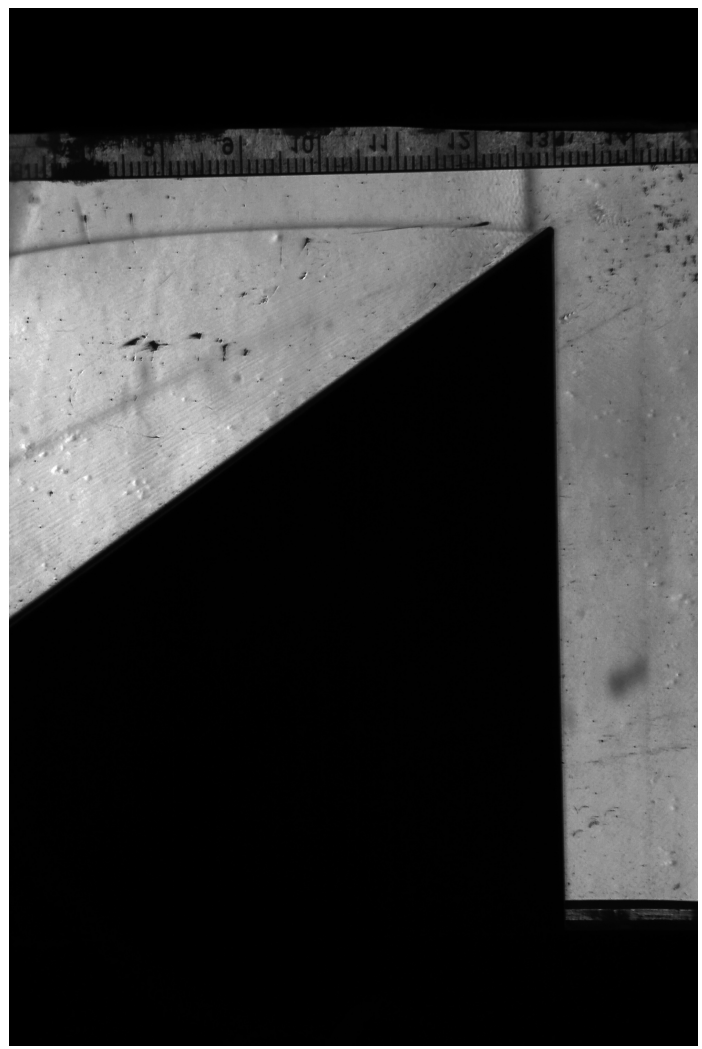

Figure 3-10: Detonation wave in $\mathrm{C}_{2} \mathrm{H}_{2}+2.5 \mathrm{O}_{2}$ at $20 \mathrm{kPa}$ over $35^{\circ}$ wedge

However it was demonstrated previously that the cell size is important for Mach reflection process. From Fig. 3-11 a Mach stem is observed over the $35^{\circ}$ wedge when the initial pressure is reduced to $5 \mathrm{kPa}$. Thus the critical angle of transition varies for a given mixture depending on the cell size of the incident detonation. 


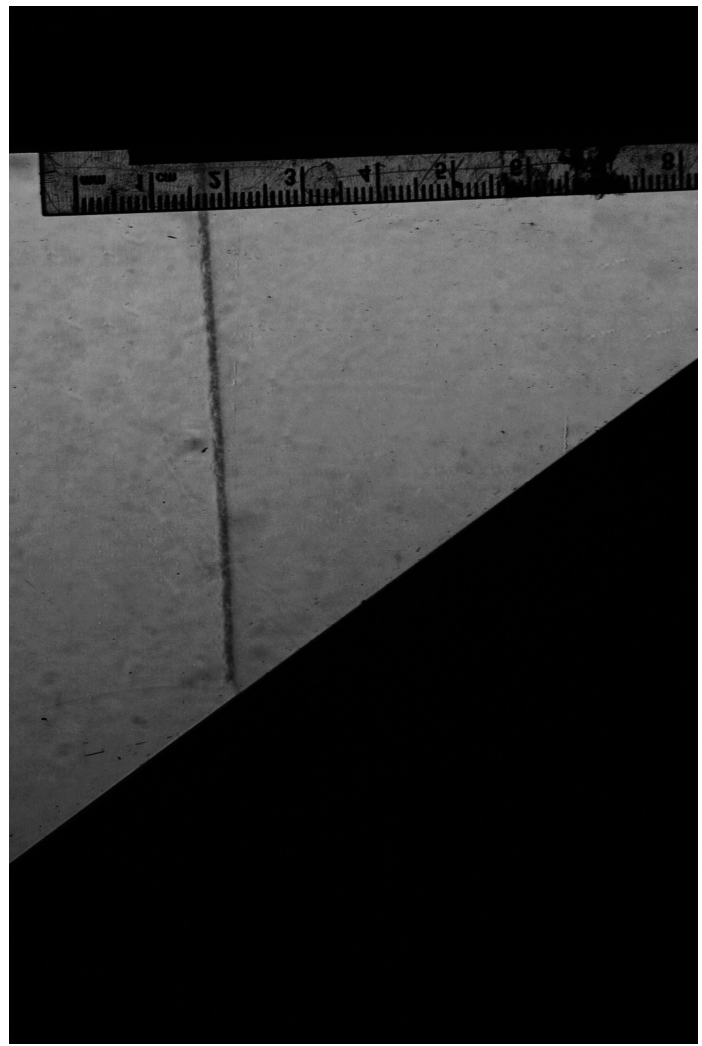

Figure 3-11: Detonation wave in $\mathrm{C}_{2} \mathrm{H}_{2}+2.5 \mathrm{O}_{2}$ at $5 \mathrm{kPa}$ over $35^{\circ}$ wedge

\subsection{Smoke Foils}

A typical smoke foil for the $\mathrm{C}_{2} \mathrm{H}_{2}+2.5 \mathrm{O}_{2}+70 \%$ mixture at $5 \mathrm{kPa}$ over the $30^{\circ}$ wedge is shown in Fig. 3-12. 


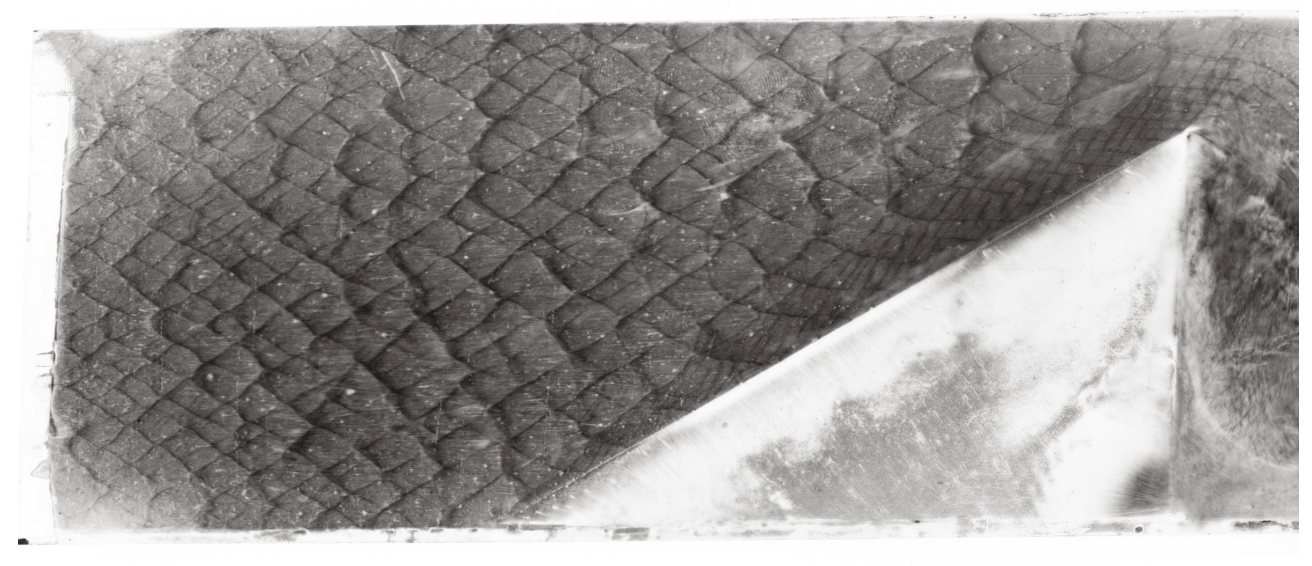

Figure 3-12: Smoke foils of a detonation in $\mathrm{C}_{2} \mathrm{H}_{2}+2.5 \mathrm{O}_{2}+70 \% \mathrm{Ar}$ at $5 \mathrm{kPa}$ over a $30^{\circ}$

In this case the fairly regular transverse waves pattern of the incident detonation can be observed. The different cell size of the overdriven Mach stem is also clearly evident. It can be seen that the boundary separating the overdriven Mach stem and the incident detonation is not a straight line. It not only appears to follow a curve, but the boundary itself seems to oscillate in a "wavy" pattern. Looking at the Mach stem region, there is evidence that the cell size varies between the near-field and the far-field region. This indicates that the Mach stem takes some time to fully develop. It is also clear that the cellular patterns are sort of superimposed on one another. Thus to properly define the triple point region one would require a detailed study of the interaction between the transverse waves of the intrinsic cellular instability and that of the reflected transverse shock of the Mach reflection process. This can be further demonstrated by looking at the more unstable mixtures of $\mathrm{C}_{3} \mathrm{H}_{8}+5 \mathrm{O}_{2}$ shown in Fig. 3-13 for an initial pressure of $5 \mathrm{kPa}$ over the $30^{\circ}$ wedge. 


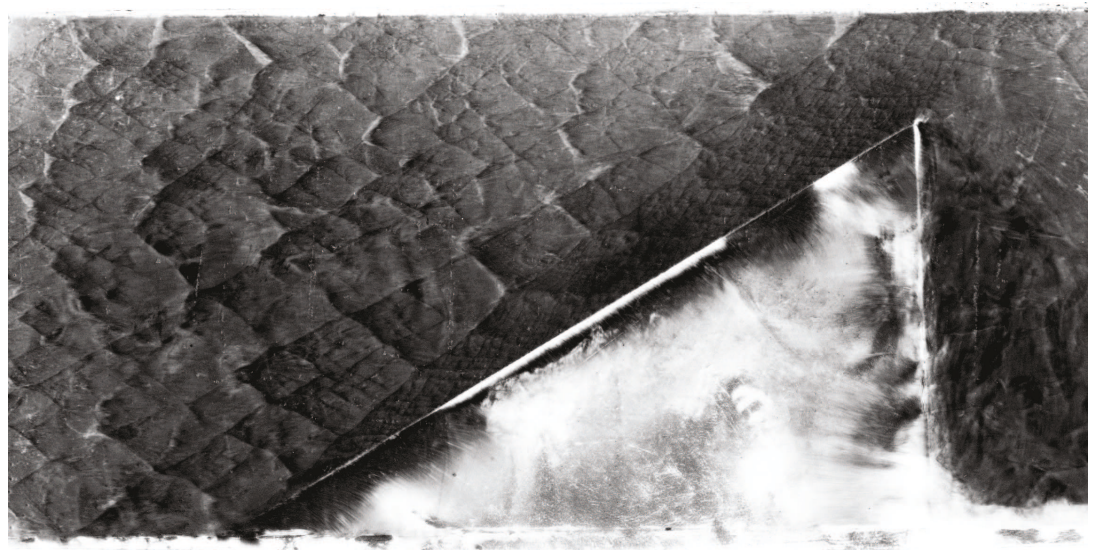

a)

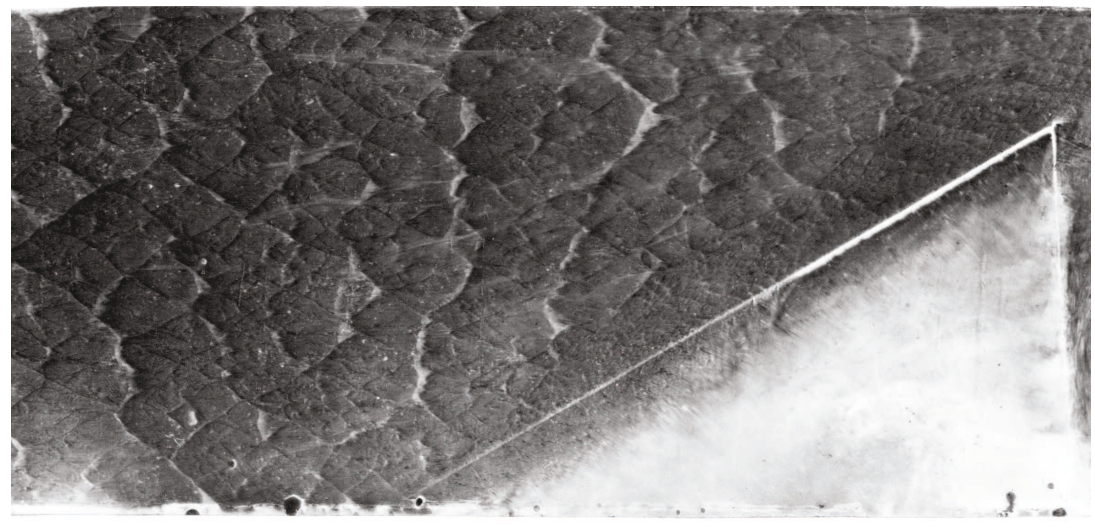

b)

Figure 3-13: Smoke foils of a detonations in $\mathrm{C}_{3} \mathrm{H}_{8}+5 \mathrm{O}_{2}$ at $5 \mathrm{kPa}$ over a $30^{\circ}$ wedge

The first observation is the fact that cellular structure is not regular. However the boundary separating the structure of the Mach stem from that of the incident detonation is quite distinct. Once again the boundary follows a curved and "wavy" path as the detonation propagates along the wedge. The cell size of the Mach stem region also appear to vary mildly throughout, but in this case the change is much harder to observe due to the irregular cellular structure inherent of this mixture. Comparing Fig. 3-13a) and b) in which the 
far-field Mach stem boundary pattern appear similar, it can be seen that the near-field is very different. The boundary remains very near the wedge surface in Fig. 3-13p) before suddenly rising to a similar height to that of the smoke foil in Fig. 3-13a). This is once again an indication that the triple point region is a consequence of the interaction between the transverse waves of the Mach stem and that of the incident detonation as well as the reflected shock of the Mach reflection.

\subsection{Graphical Results}

\subsubsection{Effect of Initial Pressure}

The height of the Mach was measured from both the schlieren photographs and the smoke foils. For the schlieren photographs the Mach stem can be measured for each individual photograph as the distance between the perceived triple point and the wedge surface. For the smoke foils, the height of the Mach stem region was determined based on the estimated boundary between the incident and Mach stem region. The results are presented alongside the calculated values of the triple point trajectory obtained from the three-shock theory for both the unreactive (shock wave case, assumed to travel at $\mathbf{M}_{c j}$ ) and reactive (thin discontinuity case). The measurement of the height of the Mach stem versus incident wave propagation distance at different initial pressures for all three mixtures, obtained from schlieren photographs are shown in Fig. 3-14. It is seen that at higher initial pressures (smaller cell sizes) the height of the Mach stem is observed to be smaller than the height measured at lower pressure (larger cell sizes). This clearly demonstrates the dependence on the initial pressure on the triple point trajectory. 


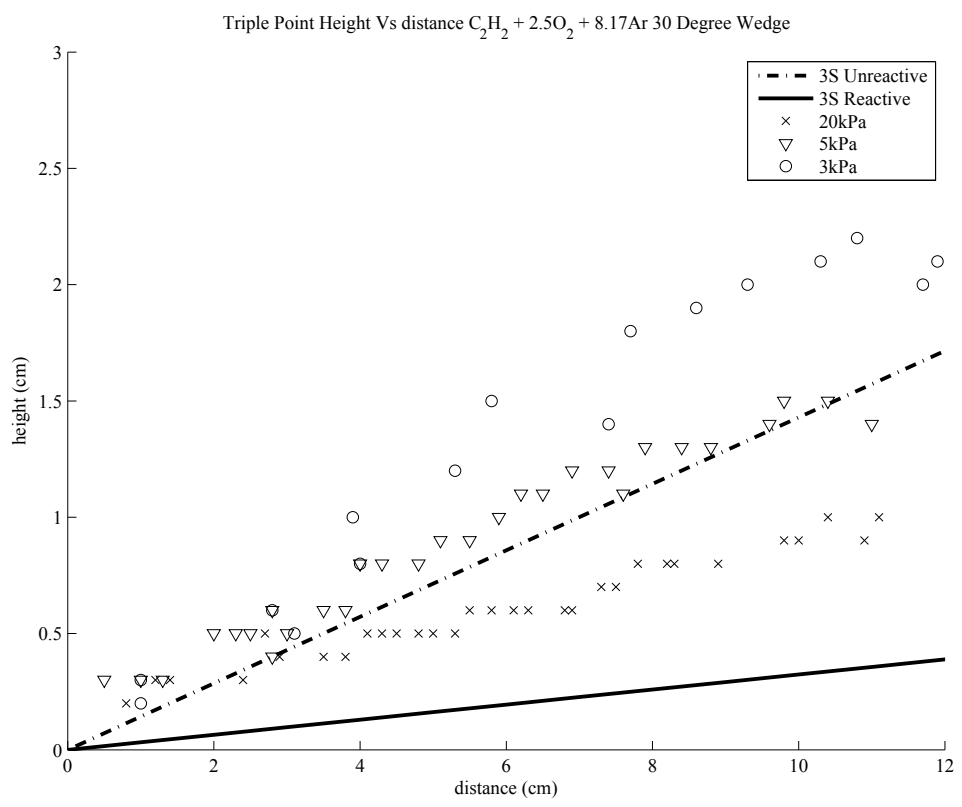

(a)

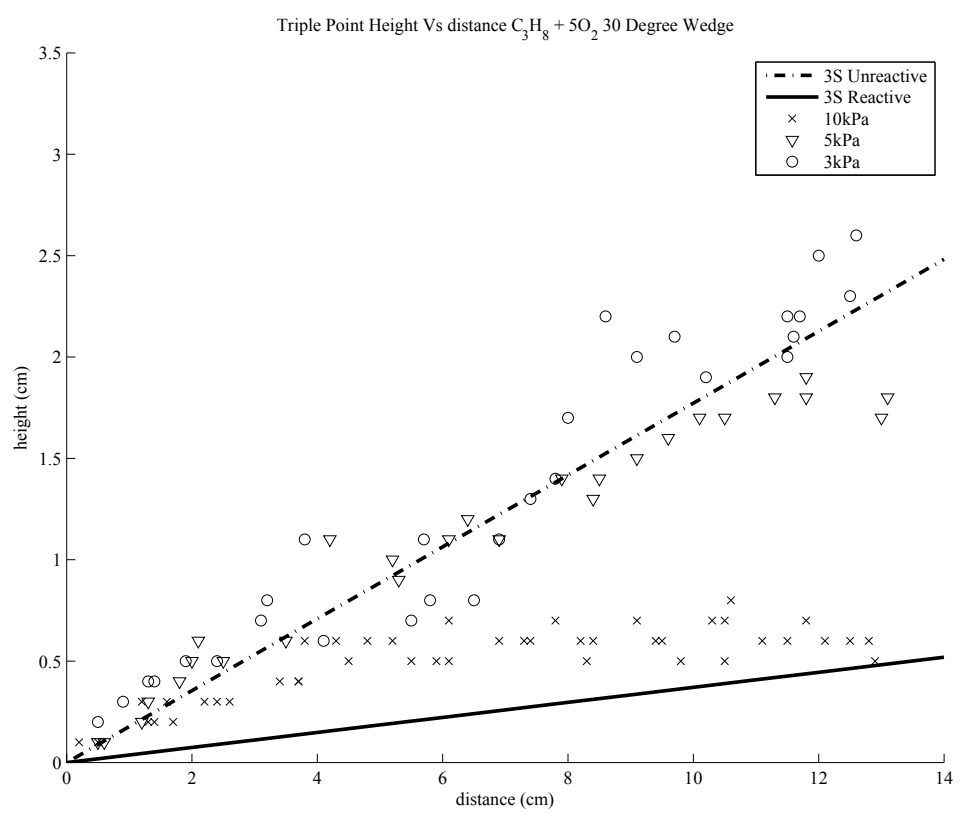

(b) 


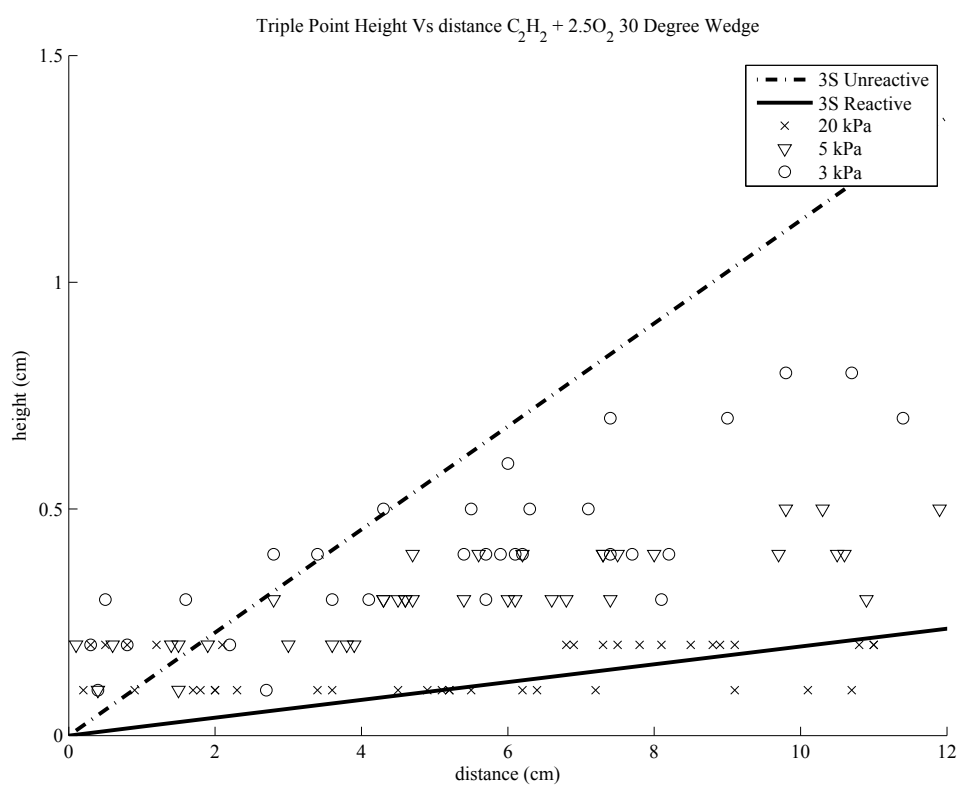

(c)

Figure 3-14: Height of Mach stem VS propagation distance for detonation waves in mixtures of a) $\mathrm{C}_{2} \mathrm{H}_{2}+2.5 \mathrm{O}_{2}+70 \% \mathrm{Ar} \mathrm{b}$ ) $\mathrm{C}_{3} \mathrm{H}_{8}+5 \mathrm{O}_{2}$ and c) $\mathrm{C}_{2} \mathrm{H}_{2}+2.5 \mathrm{O}_{2}$ at different initial pressures, over a $30^{\circ}$ wedge

At higher initial pressure (smaller cell size) the detonation front appears planar. The transverse waves behind the incident are very close to each other and their reflection from the wedge surface may play a role in the Mach reflection process. These reflected transverse waves would increase the chemical reaction rate behind the Mach stem. This would effectively reduce the cell size behind it as well as accelerate it. However if the initial pressure is low (large cell size) then the front is no longer planar. A consequence of this is that the perceived wedge angle may be different from the actual wedge angle due to the curvature of the detonation front. This would impact on the strength of the reflected wave and thus the initial formation of the Mach stem. However since detonations propagate 
based on the expansion of the product behind the wave and not on the flow-field behind it this impact would be minimal when the detonation has traveled far from the apex. As such it was not observed in this study as the focus was made to observe the Mach reflection process over the entire length of the wedge. Another aspect of larger cell size is that the transverse waves behind the incident are further apart. This would lead to a decrease in the frequency of the reflected transverse waves on the wedge. This means that the chemical reaction rate behind the Mach stem would not be increased as much as when the cell size is small.

In general the past results tend to agree more with the unreactive three-shock theory. But in previous studies wedges used are often short to accomodate the small channel height. Looking at the results presented above it can be seen that the triple point trajectory appears to follow a good agreement with the unreactive theory but only in the near-field. Thus if the wedge length is too short the Mach stem is not given enough time to properly reach steady-state which may explains why some results in the past show good agreement with the theotical results calculated by the unreactive three-shock theory. It was discussed by Guo [13] that the initial pressure had no bearings on the triple point trajectory $\chi$. This disagrees with the present results. Looking at the Mach stem height variation with initial pressure, it can be seen that the variation in the trajectory of the triple point from different initial pressures only becomes noticeable once the Mach stem reaches steady-state in the far-field. Guo used a small channel (channel height of $4 \mathrm{~cm}$ ), which means he used relatively short wedges since they were all of constant height $(3.5 \mathrm{~cm})$. In this case he would observe waves that are only in the near-field region in which the variation of Mach stem height is fairly independent on the initial pressure. 


\subsubsection{Effect of Wedge Angle and Mixture}

Looking at the schlieren photograph results for the $\mathrm{C}_{2} \mathrm{H}_{2}+2.5 \mathrm{O}_{2}+70 \%$ Ar mixture for different wedge angles from Fig. 3-15 a general trend can be seen. In the near-field there is somewhat good agreement with the three-shock unreactive solution, however as the Mach reflection approaches the far-field the Mach stem appears to stabilize and asymptotically approach a finite value. In Fig. $3-15$ b),c) and d) it appears that the slope of the trajectory in the far-field is parallel to the slope of the three-shock reactive solution. Of note, in Fig. 3-15 c) and d) the reactive three-shock solution does not appear in the graph. This is due to the fact that the reactive three-shock theory predicts a critical transition angle of about $36^{\circ}$, the trajectory angle value for the $35^{\circ}$ wedge is near $0^{\circ}$ and the trajectory angle is $0^{\circ}$ for the $40^{\circ}$ since regular reflection is expected from three-shock theory. The trajectories can be approximated as curved lines, perhaps with the exception of the $40^{\circ}$ wedge, since the Mach stem is very small for this wedge throughout the propagation. 


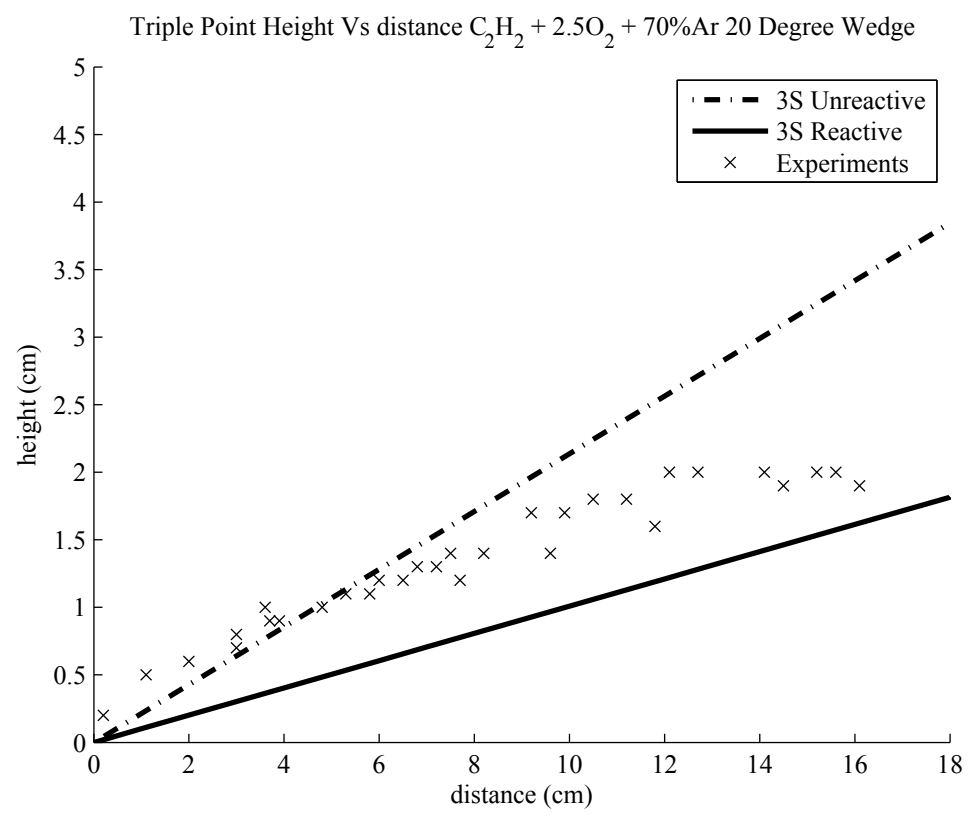

(a)

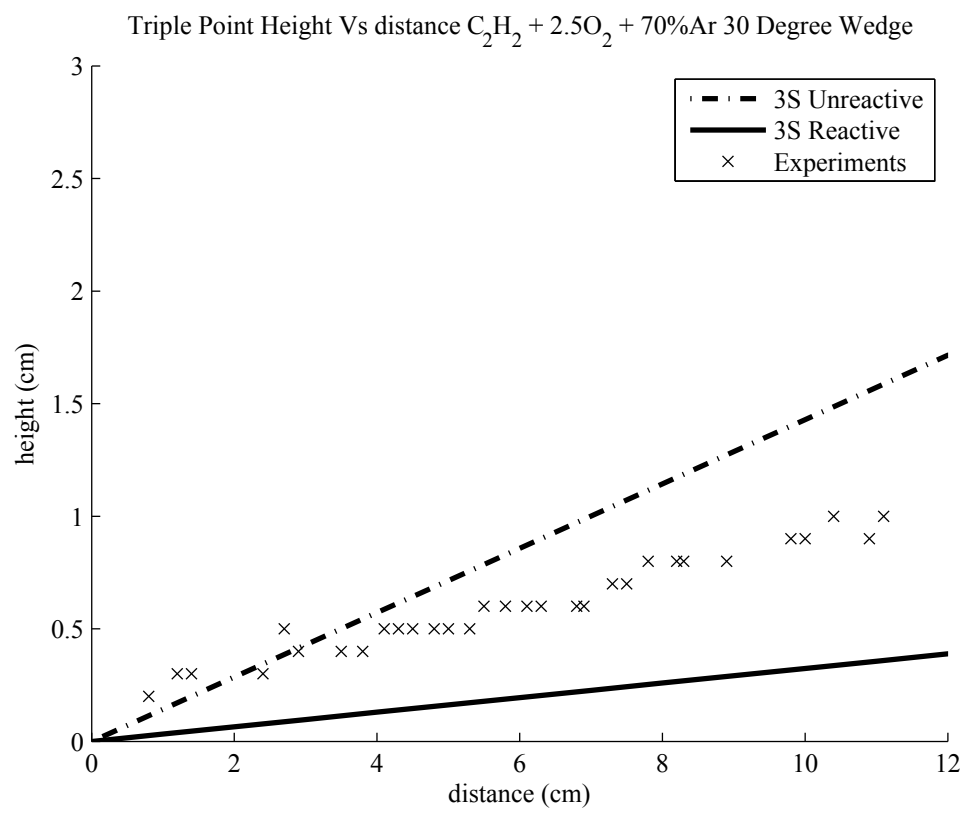

(b) 


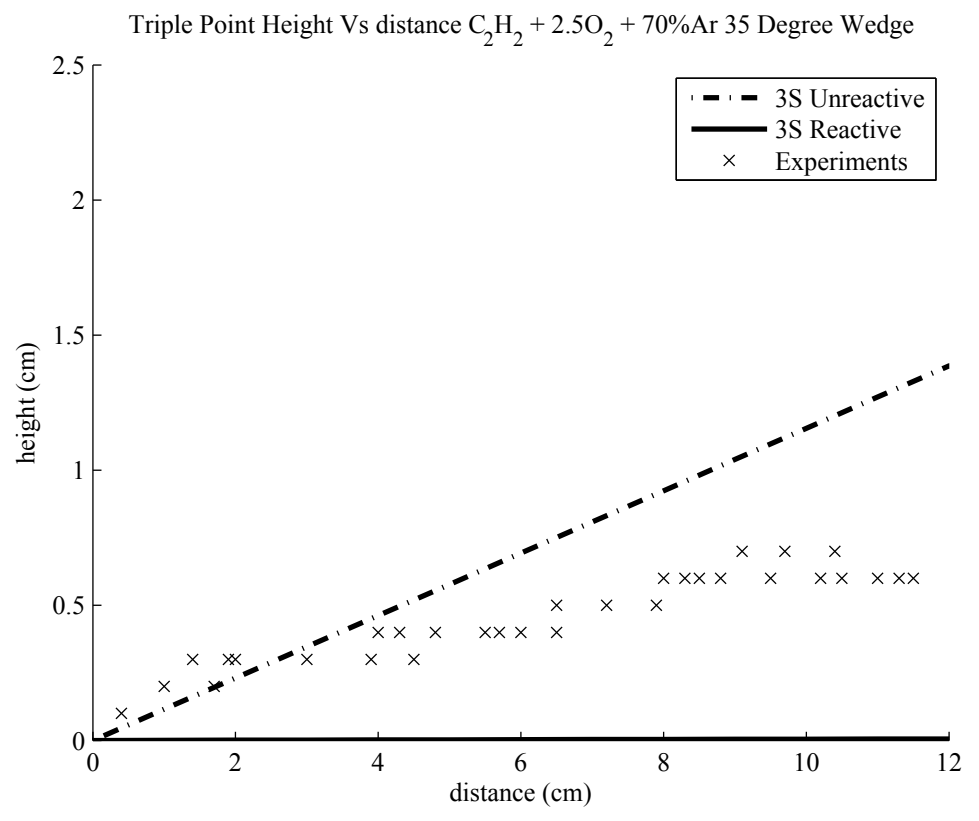

(c)

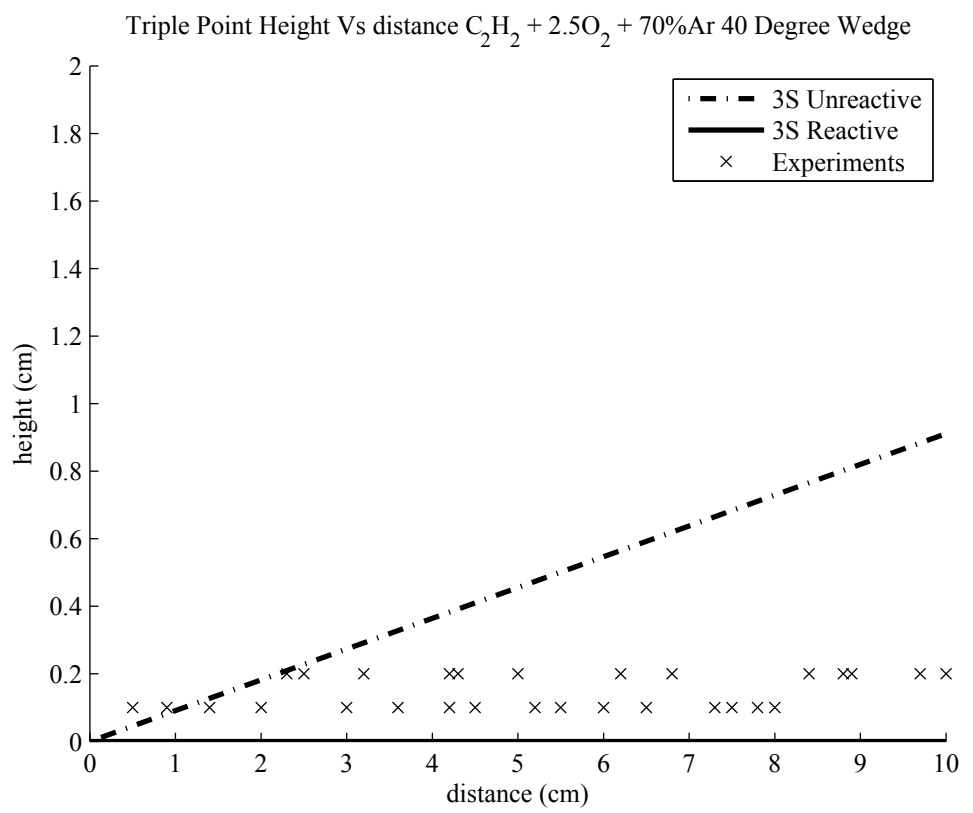

(d)

Figure 3-15: Height of Mach stem VS propagation distance for $\mathrm{C}_{2} \mathrm{H}_{2}+2.5 \mathrm{O}_{2}+70 \% \mathrm{Ar}$ at $20 \mathrm{kPa}$ over different wedges 
Comparing the results with those obtained from schlieren photographs for the $\mathrm{C}_{3} \mathrm{H}_{8}$ $+5 \mathrm{O}_{2}$ mixture from Fig. $3-16$ shows clearly that the trajectory of the triple point is not a straight line. Once again the results are in agreement with the three-shock unreactive predictions in the near-field. Further downstream the Mach stem appears to have reached an asymptotic value. Although the asymptotic value approached appears to be parallel with the $0^{\circ}$ trajectory of the reactive predictions in the $35^{\circ}$ and $40^{\circ}$ wedge it is not so for the $20^{\circ}$ wedge. The reason for this problem is currently not known but perhaps it is due to potential scatter of the data. From the smoke foils it was shown that the triple point boundary oscillated alongside the incident wave trajectories. This increases the scatter of the measurement in the case of the propane which yields irregular cell structure. 


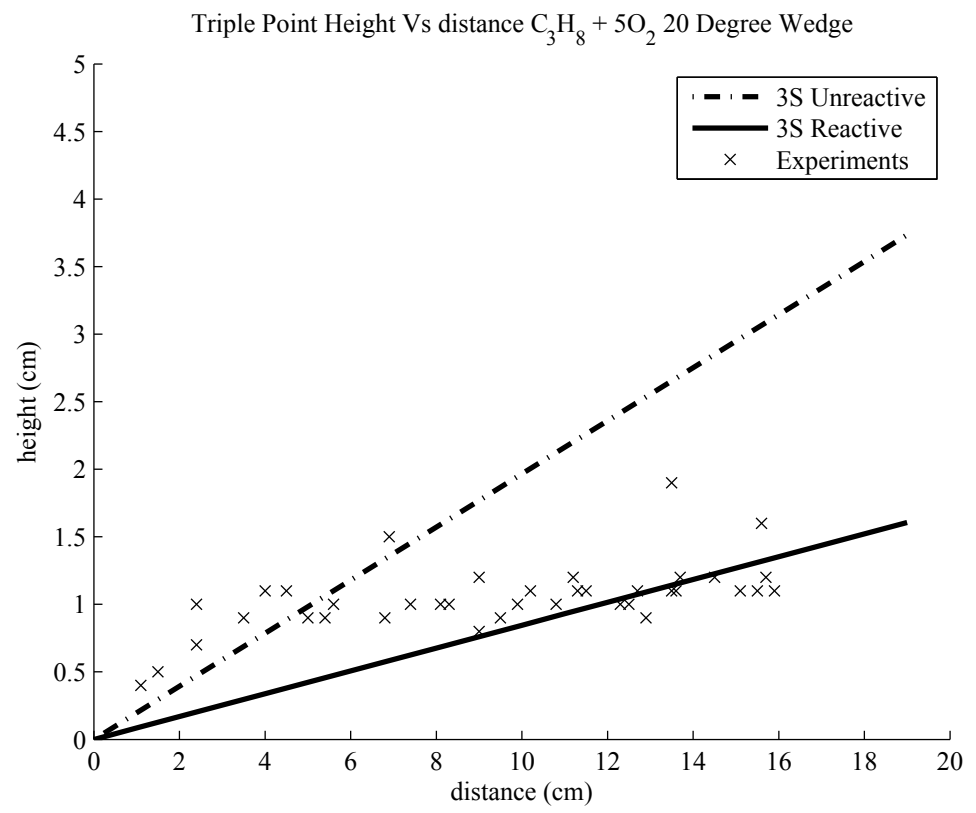

(a)

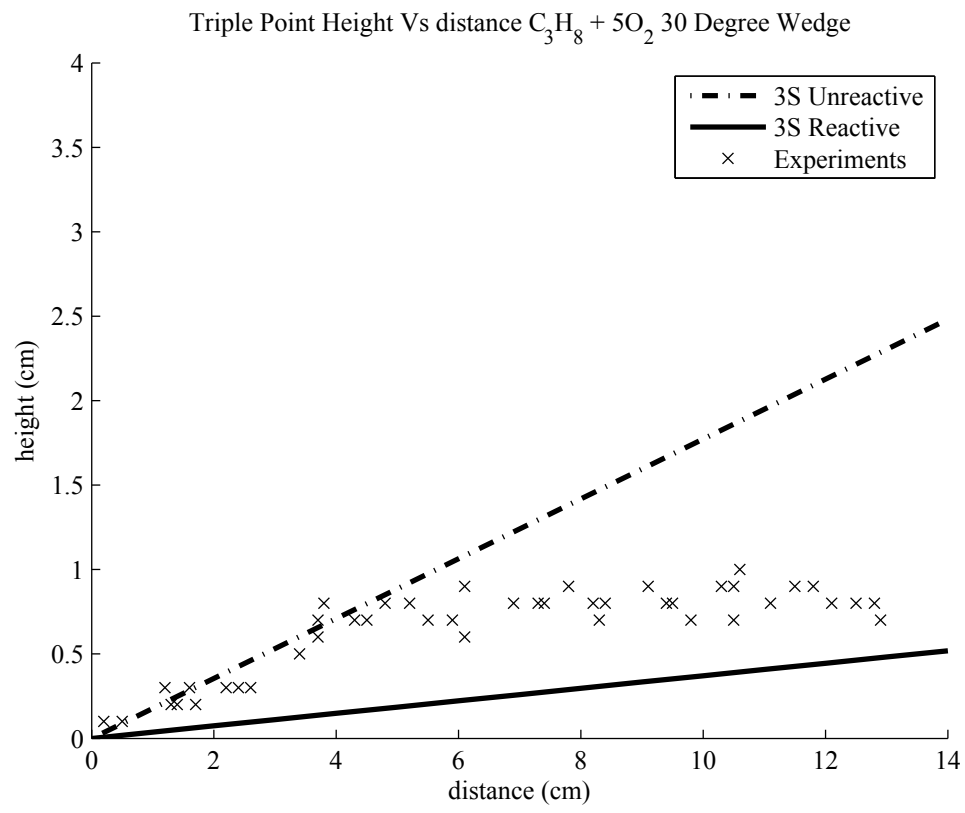

(b) 


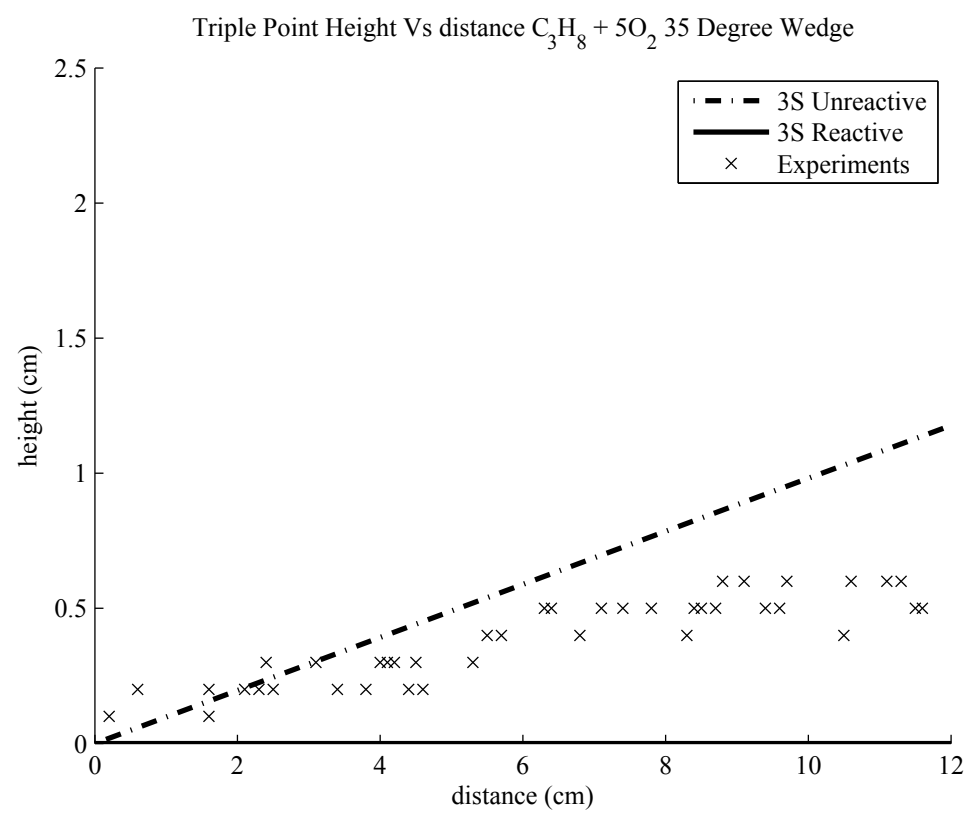

(c)

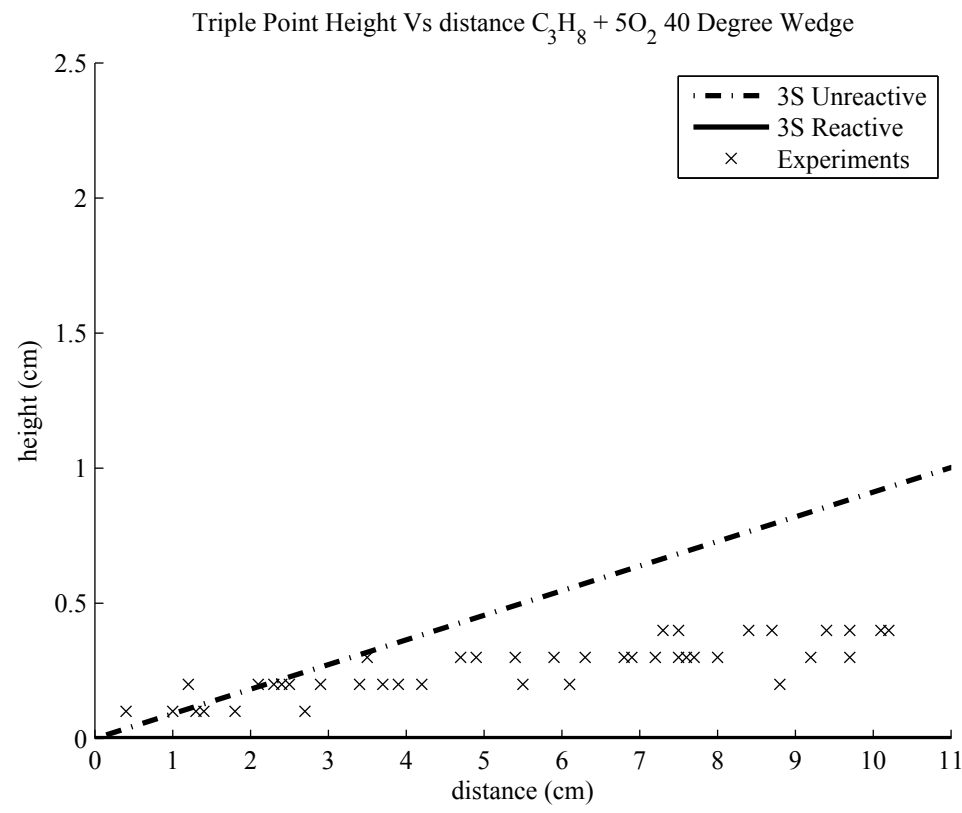

(d)

Figure 3-16: Height of Mach stem VS propagation distance for $\mathrm{C}_{3} \mathrm{H}_{8}+5 \mathrm{O}_{2}$ at $10 \mathrm{kPa}$ over different wedges 
The schlieren photographs results of the $\mathrm{C}_{2} \mathrm{H}_{2}+2.5 \mathrm{O}_{2}$ from Fig. $3-17$ fall well below that of both theoretical predictions with the smallest Mach stem height of all the mixtures. This is possibly due to the high sensitivity of the mixture. An oscillatory pattern in the triple point trajectory can be seen. Note that the $35^{\circ}$ wedge is not present because regular reflection was observed at $20 \mathrm{kPa}$. 


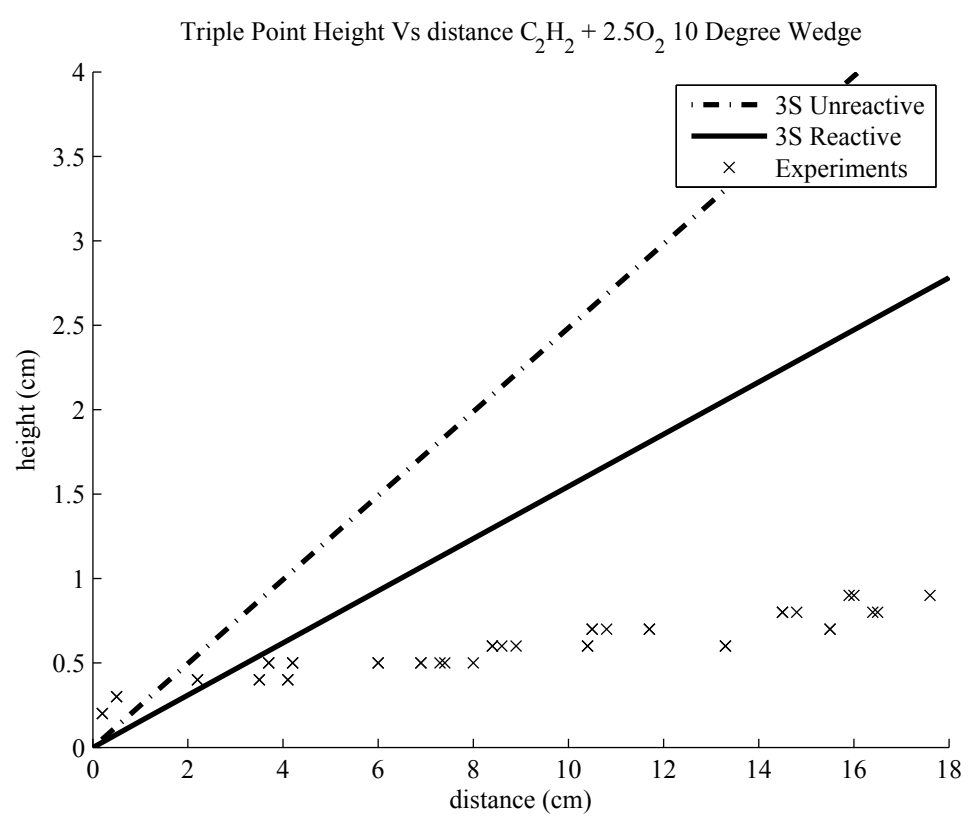

(a)

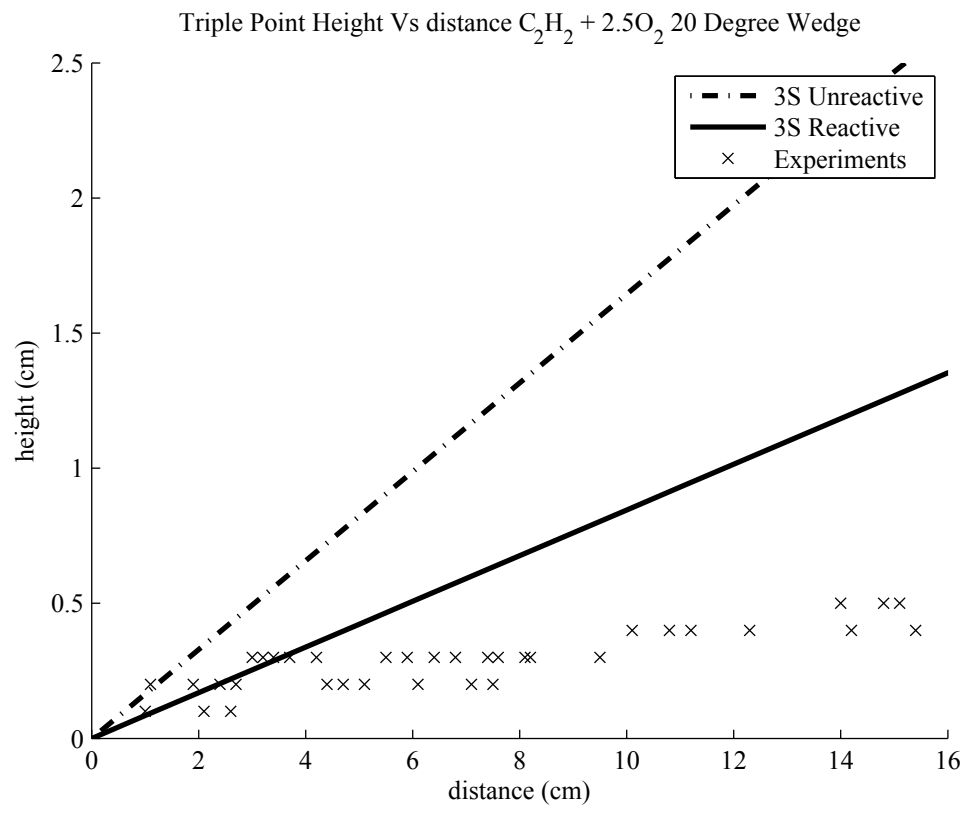

(b) 


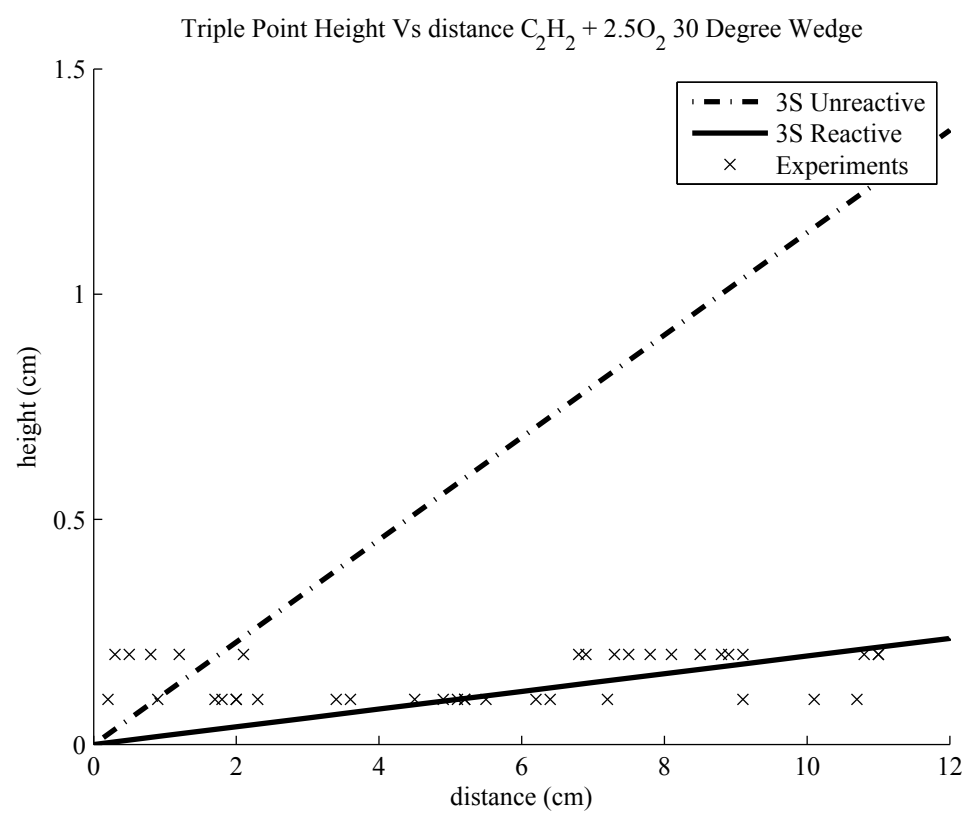

(c)

Figure 3-17: Height of Mach stem VS propagation distance for $\mathrm{C}_{2} \mathrm{H}_{2}+2.5 \mathrm{O}_{2}$ at $20 \mathrm{kPa}$ over different wedges

In past studies with smoke foils investigators have measured an angle $\chi$ by drawing a straight line across the uneven boundary between the incident detonation front and Mach stem and joining it to the wedge apex. In the case of schlieren photographs they have joined a line between the triple point and wedge apex from individual pictures. This is not adequate because it overlooks the curvature of the path exhibited by all mixtures. Akbar [1] mentioned that he obtained self-similarity within the field of view of is experiments located strictly in the far-field. However from the above results, in the far-field, the trajectory of the triple point reaches an asymptotic value, which may look self-similar. Thus simply looking at the far-field is not a good indication that the overall process is self-similar 
because it overlooks the curvature of the trajectory when observing the phenomenon over the whole wedge.

\subsubsection{Smoke foils VS schlieren}

The measurements of the height of the Mach stem region obtained from smoke foils are compared with the results obtained from the schlieren photographs in Fig. 3-18. Fig. 3-18a) and 3-18p) are for $\mathrm{C}_{2} \mathrm{H}_{2}+2.5 \mathrm{O}_{2}+70 \%$ Ar mixtures whereas Fig. 3-18c) and $3-18 \mathrm{~d}$ ) are for irregular cell pattern $\mathrm{C}_{3} \mathrm{H}_{8}+5 \mathrm{O}_{2}$ mixtures. There is more scatter obtained from the Smoke foils results (as can be seen by the spread in the 4 different shots measured) but they are in good agreement with the schlieren measurements, and thus only the schlieren measurements were shown in previous figures. It is clear that both techniques complement one other and thus permits adequate measurement to be made. The results are shown to agree with the the unreactive three-shock theory (frozen limit) in the nearfield and in the far-field, the trajectory follows a trajectory prallel to the predictions of the reactive three-shock theory (equilibrium limit). Also the triple point trajectory appears to transit between the two regimes. In spite of the fact that a precise thickness of cellular detonation front cannot be defined, the concept of frozen and equilibrium limits appear to provide a useful means to interpret the results. 
Triple Point Height Vs distance $\mathrm{C}_{2} \mathrm{H}_{2}+2.5 \mathrm{O}_{2}+8.17 \mathrm{Ar} 20 \mathrm{kPa} 30$ Degree Wedge

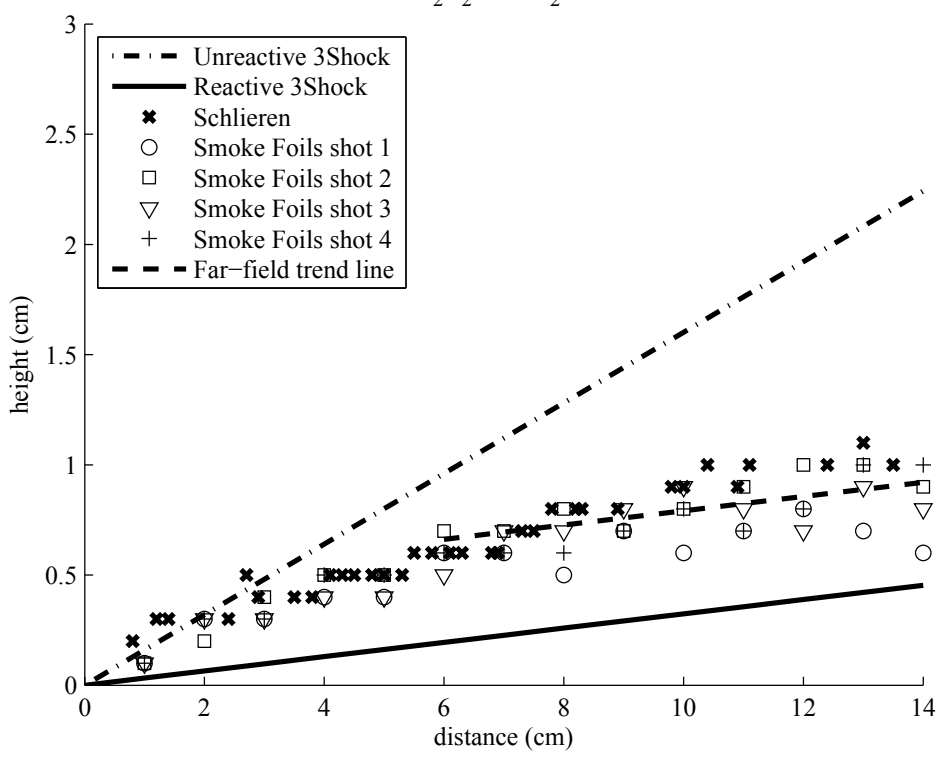

(a)

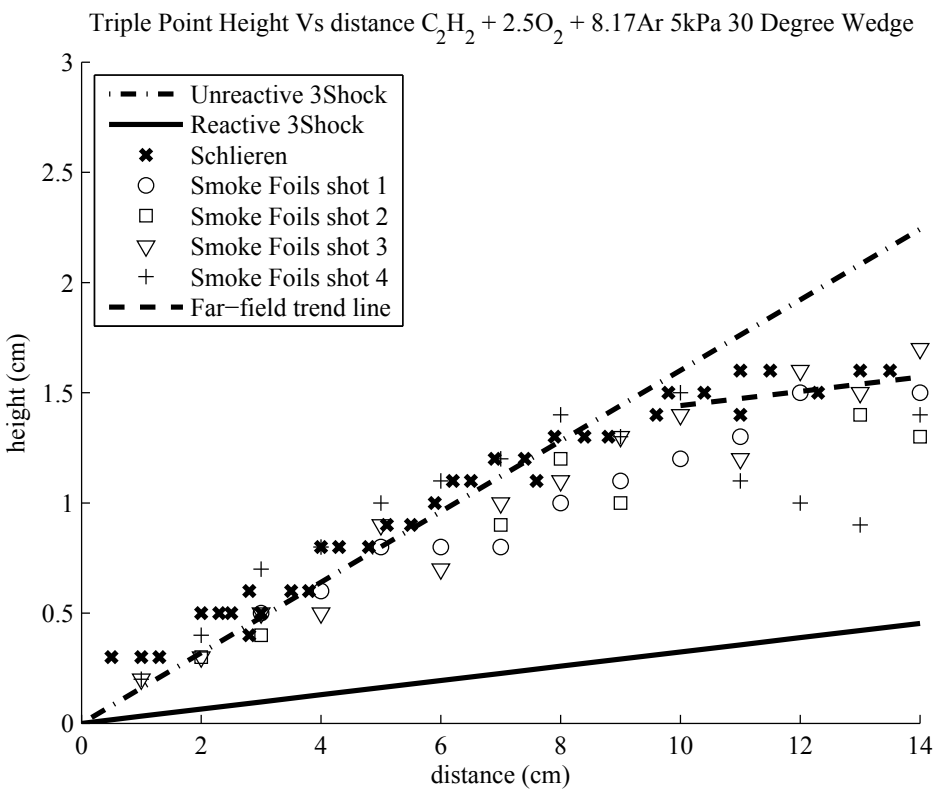

(b) 


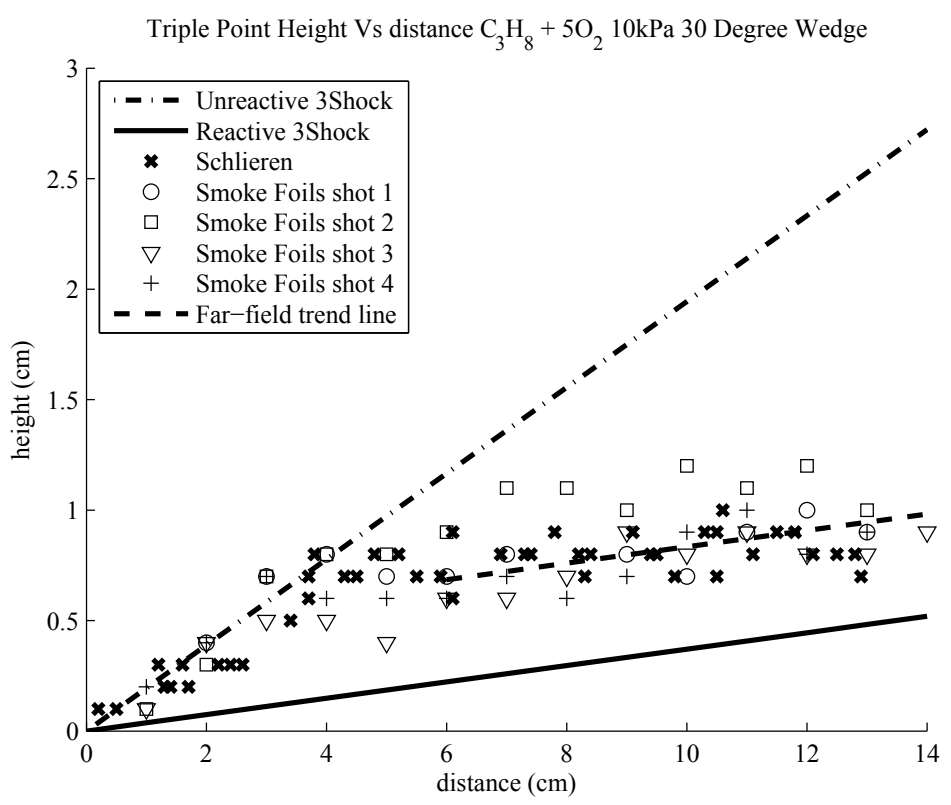

(c)

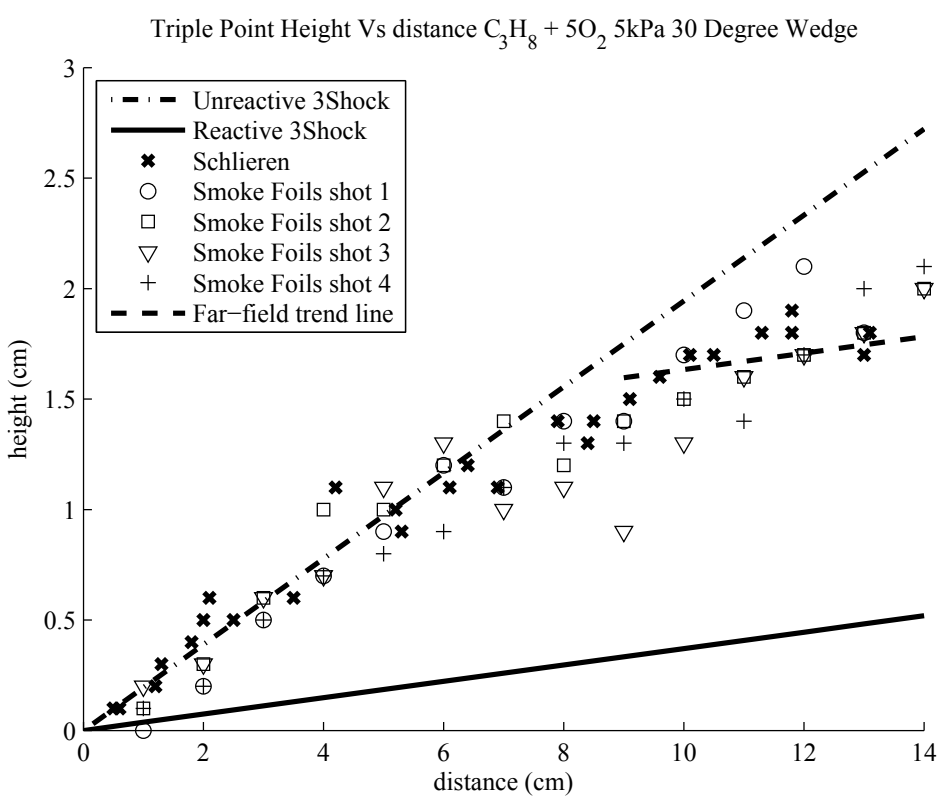

(d)

Figure 3-18: Triple point height vs distance traveled over a $30^{\circ}$ wedge for a) $\mathrm{C}_{2} \mathrm{H}_{2}+2.5 \mathrm{O}_{2}$ $+70 \% \mathrm{Ar} \mathrm{P}_{\text {ini }}=20 \mathrm{kPa}$ b) $\mathrm{C}_{2} \mathrm{H}_{2}+2.5 \mathrm{O}_{2}+70 \% \mathrm{Ar} \mathrm{P}_{i n i}=5 \mathrm{kPac}$ ) $\mathrm{C}_{3} \mathrm{H}_{8}+5 \mathrm{O}_{2} \mathrm{P}_{i n i}=10 \mathrm{kPa}$, d) $\mathrm{C}_{3} \mathrm{H}_{8}+5 \mathrm{O}_{2} \mathrm{P}_{\text {ini }}=5 \mathrm{kPa}$ 


\section{CHAPTER 4}

Conclusion

In the present investigation, the Mach stem height is determined over the entire length of the wedges. If one or two schlieren photographs are taken at some local region on the wedge and a line is joined from the apex of the wedge to the triple point observed from the photographs the detailed variation cannot be obtained and erroneous results (hence conclusions) are obtained for the triple point trajectory angle. Similarly if only a short

length of wedge is used, a straight line can be fitted to obtain a triple point trajectory angle. This again would give erroneous results. It is clear that the phenomenon requires enough time for the Mach stem to fully develop in order to be properly studied. The "scatter" in the data for the smoke foils comes from the measurements of various shots, which were shown previously to have the Mach stem boundary oscillate as the incident wave propagates, due to the interaction of the between the reflected wave, the incident wave transverse waves and the Mach stem transverse waves. Thus every shot is unique but a general trend can still be established.

The current investigation confirms the failure of the three-shock theory prediction of the triple point trajectory in the Mach reflection of cellular detonations, if one takes the definition of self-similarity strictly, ie the triple point trajectory is a straight line from the wedge apex. This is due to the presence of a length scale that characterizes a detonation front. Although the failure of self-similarity was observed by other investigators previously, they have persisted in comparing their results (experimental and numerical) 
with the self-similar three-shock theory which led to confusion. In many of the previous investigations, the triple point trajectory angle was obtained by either fitting a straight line through the irregular boundary between the incident wave and the Mach stem region. They fit a line through the observed triple point to the wedge apex for individual schlieren pictures. Furthermore the Mach reflection was observed at one localized position on the wedge (or over very small wedges). This results in the complex nature of the problem not being properly revealed. The present work has shed some light on the nature of the transverse waves interactions at the triple point indicating that a proper description of the Mach reflection of cellular detonations requires the detailed analysis of the transient wave interaction processes between the Mach stem and those of the incident detonation front.

On the other hand if one considers a small distance from the wedge apex or a region in the far-field when the distance of propagation of the Mach stem is large compared to the cell size, self-similar behavior is indicated. Thus it may be concluded that the frozen equilibrium limits do offer a useful approximate means to describe the Mach reflection phenomenon.

The past conclusion that the initial pressure had no impact on the triple point trajectory was also proven to be incorrect. A reduction of the initial pressure was shown to increase the triple point trajectory for mixtures with both a regular and irrefular structure. This also applied to the critical angle of transition to regular reflection. Only the $\mathrm{C}_{2} \mathrm{H}_{2}+$ $2.5 \mathrm{O}_{2}$ mixture at initial pressure of $20 \mathrm{kPa}$ was shown to transit to regular reflection over the $35^{\circ}$ wedge. Whereas the other mixtures did not transit even over a $40^{\circ}$ wedge. The change in initial pressure is equivalent to a change in cell size, thus it further demonstrates the high dependence of the transverse wave interaction near the triple point region. 
Based on the smoke foils it was observed that the boundary between the Mach stem and incident wave regions is not only curved but also irregular. The cellular structure behind the Mach stem appears to show a transition region between the formation of the Mach stem and when it reaches steady-state in the far-field. More studies are required with smoke foils on the wedge surface to investigate the development of the Mach stem and see if the curved trajectory of the triple point matches the Mach stem stabilizing into steady-state conditions. 


\section{References}

[1] Raza Akbar. Mach reflection of gaseous detonations. PhD thesis, 1997.

[2] T.V. Bazhenova, L.G. Gvozdeva, Yu. S. Lobastov, L. M. Naboko, R.G. Nemkov, and Predvoditeleva O.A. Shock waves in real gases. 1968.

[3] W. Bleakney, C. H. Fletcher, and D. K. Weimer. The density field in mach reflection of shock waves. Phys. Rev., 76:323-324, Jul 1949.

[4] Walker Bleakney and A. H. Taub. Interaction of shock waves. Rev. Mod. Phys., 21:584-605, Oct 1949.

[5] V.V. Mitrofanov B.V. Voitsekhovskii and M.E. Topchian. The structure of a detonation front in gases. Wright-Patterson Air Force Base Report.

[6] L. Hamada D. Desbordes, C. Guerraud and H.N. Presles. Failure of the classical dynamic parameters relationships in highly regular cellular detonation systems. Progress in Astronautics and Aeronautics, 153:347.

[7] J.R. Walker M.A. Nettleton Edwards, D.H. On the propagation of detonation waves along wedges. Archivum Combustion, 4:197.

[8] E.S. Prokhorov Gavrilenko, T.P. Overdriven gaseous detonations. Shock Waves, Explosions and Detonations: Progress in Astronautics and Aeronautics, 87:244.

[9] E.S. Prokhorov Gavrilenko, T.P. Compressed detonation wave in a real gas. Combustion, Explosion and Shock Waves, 17:689-692, 1982.

[10] Y.A. Nikolaev M.R. Topchiyan Gavrilenko, T.P. Supercompressed detonation waves. Combustion, Explosion and Shock Waves, 15:659-692, 1980.

[11] J.S. Grondin. The onset of detonation downstream of perforated plates. Master's thesis, 2010.

[12] R.A. Gross. Oblique detonation waves. AIAA Journal, 1:1225-1227, 1963. 
[13] C. Guo, D. Zhang, and W Xie. The mach reflection of a detonation based on soot track measurements. Combustion and Flame, 127, 2001.

[14] O.A. Predvoditeleva Gvozdeva, L.G. Triple configurations of detonation waves in gases. Fizika Goreniya i Vzryva, 5:451.

[15] H. Hornung. Regular and mach reflection of shock waves. Annual Review of Fluid Mechanics, 18:33-58, 1986.

[16] HG Hornung, H Oertel, and RJ Sandeman. Transition to mach reflexion of shock waves in steady and pseudosteady flow with and without relaxation. Journal of Fluid Mechanics, 90(03):541-560, 1979.

[17] S. Hoshi, T. Obara, T. Yoshihashi, and S. Ohyagi. Experiments on reflections of detonation waves by wedge. Presented at the 17th International Colloquium on Dynamics of Explosions and Reactive System, 1999.

[18] S.B. Murray J.E. Shepherd, I.O. Moen and P.A. Thibault. Analyses of the cellular structure of detonations. Symposium (International) on Combustion, 21:1649.

[19] CK Law. Diffraction of strong shock waves by a sharp compressive corner. Technical report, DTIC Document, 1970.

[20] H. Li, G. Ben-Dor, and H. Grnig. Analytical study on the oblique reflection of detonation waves. AIAA Journal, 35:1712-1720, 1997.

[21] J.and J.E. Shepherd Meltzer, R. Akbar, and A. Sabet. Mach reflection of detonation waves. Progress in Astronautics and Aeronatics, 153:78.

[22] J.H.S. Lee M.I. Radulescu, H.D. Ng and B. Varatharajan. The effect of argon dilution on the stability of acetylene/oxygen detonations. Proceedings of the Combustion Institute, , 29:2825.

[23] M.A. Nettleton. Gaseous detonations: Their nature and control.

[24] G. Thomas D. Bjerketvedt O. Moen, A. Sulmistras and P.A. Thibault. Influence of cellular regularity on the behavior of gaseous detonations. Progress in Astronautics and Aeronautics, 106:220.

[25] Shigeharu Ohyagi, Tetsuro Obara, Fusao Nakata, and Shintaro Hoshi. A numerical simulation of reflection processes of a detonation wave on a wedge. Shock Waves, 10(3):185-190, 2000 
[26] R.S.B Ong. On the Interaction of a Chapman jouget Detonation Wave with a Wedge. $\mathrm{PhD}$ thesis.

[27] J Sandeman, A Leitch, and H Hornung. The influence of relaxation on transition to mach reflection in pseudosteady flow. In Shock Tubes and Waves, volume 1, pages 298-307, 1980.

[28] G.S. Settles. Schlieren and shadowgraph techniques. Springer, 2006.

[29] J.E. Shepherd. Detonation in gases. Proceedings of the Combustion Institute, 32:83.

[30] J.E. Shepherd, E. Schultz, and R. Akbar. Detonation diffraction. Graduate Aeronautical Laboratories, 1999.

[31] GO Thomas and R Ll Williams. Detonation interaction with wedges and bends. Shock Waves, 11(6):481-492, 2002.

[32] John von Neumann. Oblique reflection of shocks, 1943.

[33] C.J. Wang and C. Guo. Infulence of low initial pressure and detonation randomness on mach reflection of gaseous detonation wave. Combustion and Flame, 2009.

[34] G.B. Whitham. A new approach to problems of shock dynamics. part 1. two dimensional problems. Journal of Fluid Mechanics, 2:146. 


\section{APPENDIX A schlieren Setup}

schlieren photography is a powerful technique that allows for the visualization of density gradients in all media, be they gases, liquids or solids. It is known that light refracts when it travels through a medium of varying density. The deflection of the light can be converted by the schlieren into dark and bright spots on the image projection plane, in our case the camera sensor. The level of darkness or brightness is related to the direction and intensity of the density gradient of the medium in which the light passes through. Because of its cost-effectiveness and ease of use post-setup, schlieren photography has become a staple in the gas dynamics community as a way to visualize real gas phenomenon. This section will discuss the present Z-type configuration and its setup.

\section{Image Formation}

Before any further discussion about the interpretation of the images the author would like to suggest the reading of the book on schlieren by G.S. Settles [28] for a very thorough explanation of the setup of different types of schlieren as well as the limitations and optimizations for them. In this section only the basics will be covered. Schlieren systems can use either a point light source or an extended light. 
a)

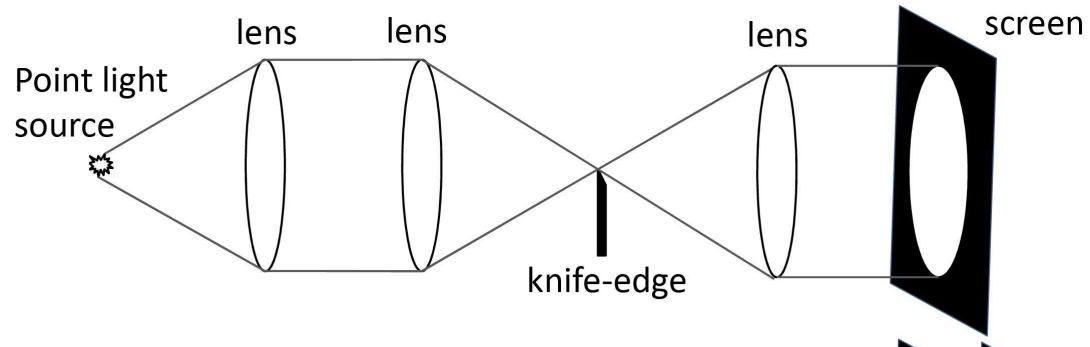

b)

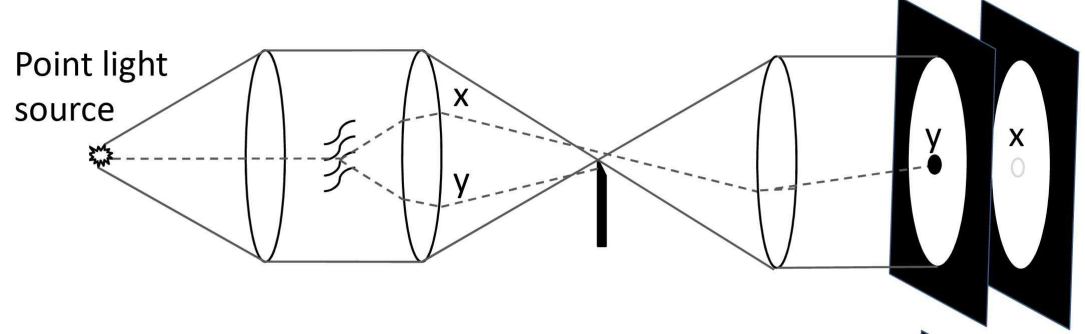

c)

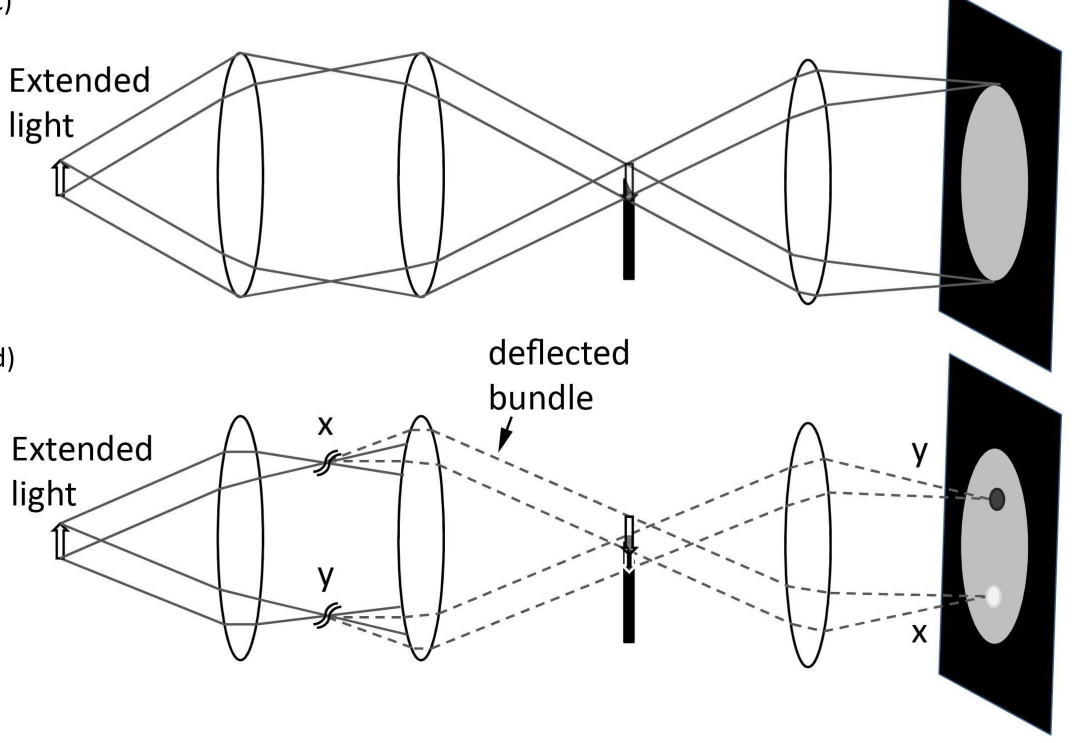

Figure A-1: Simple schlieren a), b)with point light source and c), d) extended light source

Referring to Fig. A-1 we can see that the point light source needs to be collimated by a lens in order to obtain a region or parallel beam of light that have homogeneous refractive index. A second lens is used to refocus the light into an image of the point source. We introduce a sharp knife edge at the focus of the second lens such that the screen behind 
remains fully illuminated. In order to obtain a true image of the point source on the screen plane we use a 3rd lens. If we add a schlieren object (for example a candle plume) in the homogeneous region, or test area, as seen in Fig. $\mathrm{A}-1 \mathrm{~b}$ ) we can see that the light will be diffracted from the original path, the direction depending in the density gradient direction. This deviation will results in different images on the screen. For example, ray $x$ gets deflected upwards. The second lens then returns it towards the knife edge, however since the ray is no longer coming normal to the lens it will not hit the focus point and will create a bright spot on the screen. Similarly ray $y$ is deflected downwards and the lens bends the ray back towards the knife edge, however it now hits the blade and in this case we have a black spot on the screen. This method is simple but shows that only density gradients in the vertical direction would be observed. Objects with horizontal component of density gradients will remain invisible on the screen. A point light source system is also very sensitive, yielding either bright or black spots in what is called on-oroff fashion. Most light systems however are considered to be an extended source because it is near impossible to obtain a pure point light source of infinitesimal size. Fig. A-1c) shows such a configuration. If we look at the extended light source to be a series of many point sources along a vertical axis we can see that each point source would contribute to illuminate the screen and every point in the schlieren field receives light from every point source in the extended light. This leads to the conclusion that the image formed at the focus of the second lens is that of the composite light source. The knife edge, if well placed, will cut the beam of light passing through the focus in such a way as to block out some of the point sources completely but not all of them. Since every point source helps to illuminate the screen having a knife edge will diminish the amount of light, resulting in 
a uniformly darker projection on the screen, rather than a black half followed by a bright one. In this case, if we introduce a schlieren object as shown in Fig. A-1d), we see that rays passing through are deflected and have their elemental image on the knife edge plane. Each elemental image is displaced relative to the composite light-source image which then yields either a brighter spot or a darker spot on the screen.

\section{Present configuration}

There are many ways to setup a schlieren field. Some using only lenses, some using a single or double pass through a concave mirror etc. (G.S. Settles shows many configurations in his book). The one used in this study and that will be discussed here is the Z-type configuration. The reasons being that it is compact, versatile and fairly easy to assemble. Refer to Fig. A-2 for a full setup schematic.

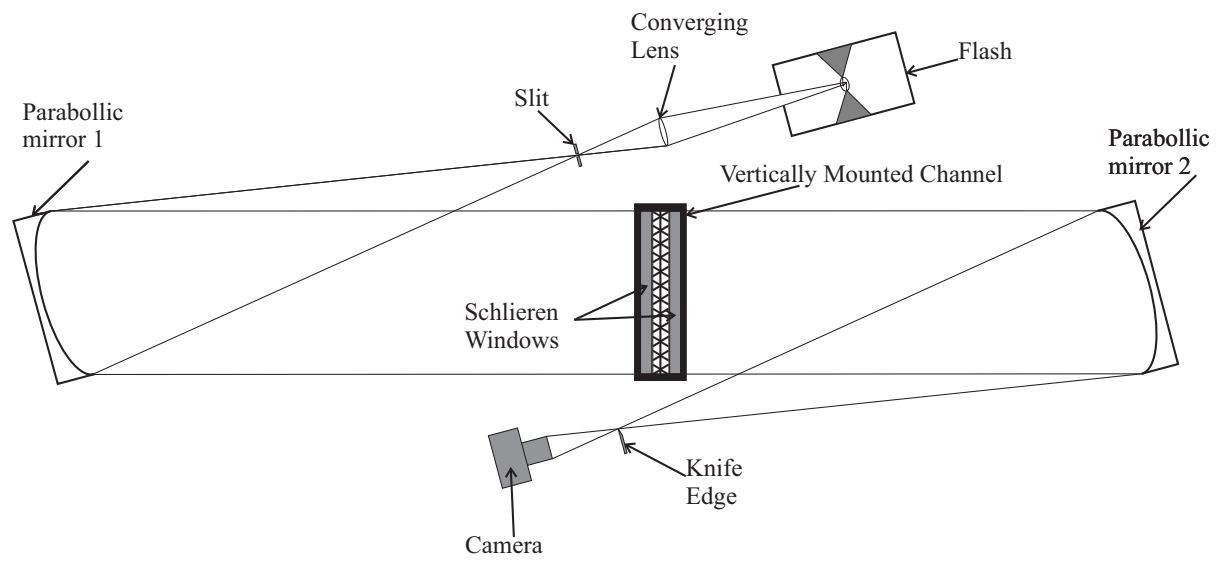

Figure A-2: Z-type schlieren configuration used

The first component in the setup is the light source. The light source used for most experiments is a spark flash (PALFLASH model 500). It was used because of its very short spark duration, spark brightness and colour. In the setup phase however a tungsten arc lamp was used because it provided a constant beam of light during mirror alignment. 
Following the light source is the condenser lens which is used to create an image of the light source on the slit. The condenser lens is placed $25 \mathrm{~cm}$ away from the light source and has a focal length of $88 \mathrm{~mm}$. The short focal length of the condenser means that unfortunately some of the light is lost at the first mirror due to the large cone of light rays that is created from this lens far away from the focus. Fig. A-3 demonstrate this problem in more details. As we can see a long focal length allows for more light to be directed at the mirror, however the setup requires more space. It was found that the flash is bright enough that some light loss is acceptable at this location and since we have severe space constraints in the laboratory it was deemed an acceptable compromise.

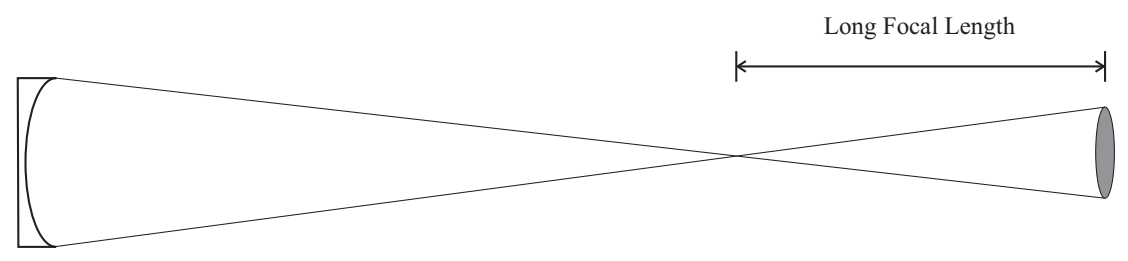

(a) Long Focal Length

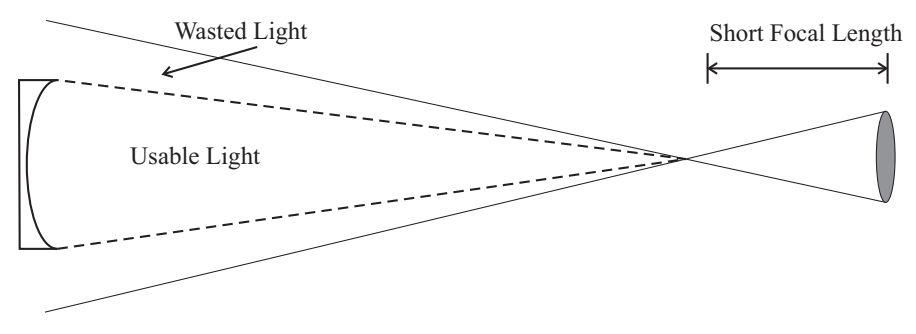

(b) Short Focal Length

Figure A-3: Condenser lens setup

The light source image is focus on the slit $8.8 \mathrm{~cm}$ away from the condenser lens. The slit is compromised of 4 razor blades arranged such that a rectangular hole of $2 \mathrm{~mm} \mathrm{x}$ $1 \mathrm{~mm}$ is formed. This is used to trim the light and increase the sharpness of the edges. 
All components so far were mounted on an optical rail measuring $1.5 \mathrm{~m}$ sitting on a heavy stand. The first mirror, which is concave, spherical and first surface finish, is located one focal length away from the slit opening, or $101 \mathrm{~cm}$ and produces a collimated beam of light. It is important to place the mirror exactly one focal length from the slit or else the beam of light will not be parallel. The mirror is angled 7 degrees away from the incoming light. This goes further than the recommended limits of 6 degrees by Settles, but it did not greatly affect the pictures. It was done because unfortunately the mirrors have relative small focal lengths for their size and the beam of parallel light was hitting the PALFLASH casing which is large. It was deemed more important to not obstruct the beam of light. If the beam of light is perfectly parallel, the light beam from the first mirror should be just large enough to completely fill the second mirror. In order to check if the beam is parallel throughout the test section one can use a sheet of paper with a circle the size of the mirrors drawn on it and check the beam at different locations. Once the beam is parallel and it fills the second mirror (which is identical to the first one), it can be turned by the exact amount in the opposite direction from the first mirror. Both mirrors are secured on heavy stands as well. One focal length away from the second mirror is where the knife edge should be positioned, such that one should be able to see an image of the rectangular slit focused on it. The experimenter should decide now which density gradient he wishes to investigate. As was discussed above the knife edge orientation changes the density gradient observed. An horizontal knife edge would allow visualization of the vertical gradients and vice-versa. In this experiment the schlieren objects moves vertically downward but the horizontal density gradients of the objects were of interest and therefore the knife edge was positioned horizontally such that the vertical density gradient were 
observed for the vertically moving object which, in the object frame of reference gave the horizontal gradients. The location of the knife edge one focal length away from the second is very important for the quality of the photographs and therefore the knife edge was mounted on an optic plate with a built-in micrometer adjuster in the longitudinal direction. Finally the camera is mounted immediately behind the knife edge in order to capture as much light as possible. The camera is a Nikon D40 with a DSLR sensor in which a $180 \mathrm{~mm}$ focal length lens with the focus set to infinity was used. Based on this we could find the location of the focus of the camera in the test area and mount our experiment there. This setup gives us a field of view $10 \mathrm{~cm}$ by $8 \mathrm{~cm}$ which allows us to see the whole width of our channel. The camera is also mounted with the knife edge on an optical rail attached to a heavy stand. Even though some light was wasted at the first mirror, it was found that the flash light was about 10 times too bright for the camera sensor. The sensor of type APS-C (Advanced Photo System type-C) is very sensitive in the range of wavelengths $475-525 \mathrm{~nm}$ which is in the range of wavelength produced by the PALFLASH and hence the camera was saturated and the quality of the picture was diminished. To prevent this it was decided to reduce the optical density down to 1 by using a neutral density filter that is designed to reduce light intensity ten-folds. It was positioned close to the knife edge in such a way as to grab all the light near the focus of the second mirror as to avoid bright rings that would form on the pictures if the filter was placed in a location where the beam of light was larger than the size of the filter. 


\section{Obtaining a picture}

In order to trigger the flash at a specific delay it was mentioned above that a digital delay generator was used and set prior to each experiments. For repeatability it was decided to not setup the delay generator based on the igniters because the detonation wave does not fully develop at the exact same location for every shot and therefore if we were to use the ignition as our zero delay the wave would appear randomly in the field of view. Instead an optic fiber located far downstream of the igniters but slightly before the wedge was used. The optic fiber connects to a photoprobe (IF-D950C). The photoprobe was powered by an external power supply of $9 \mathrm{~V}$. The circuit of the photoprobe is found in fig. A-4 and it generates a positive square pulse with $9 \mathrm{~V}$ amplitude with a pulse width that depends on the duration of time the photoprobe is exposed to light. In our case this width was ranging between 1 and $3 \mathrm{~ms}$ depending on the mixture. The pulse is then sent to our Digital delay generator (Berkeley BNC model 7010) which requires a positive input ranging between $2 \mathrm{~V}$ and $10 \mathrm{~V}$. The output of the delay generator is another positive square pulse with varying amplitude between 2 and $18 \mathrm{~V}$ that is set to occur at a fixed time, set by the experimenter depending on the field of view of the camera, after the input pulse. It was set to $15 \mathrm{~V}$ to trigger the flash. 


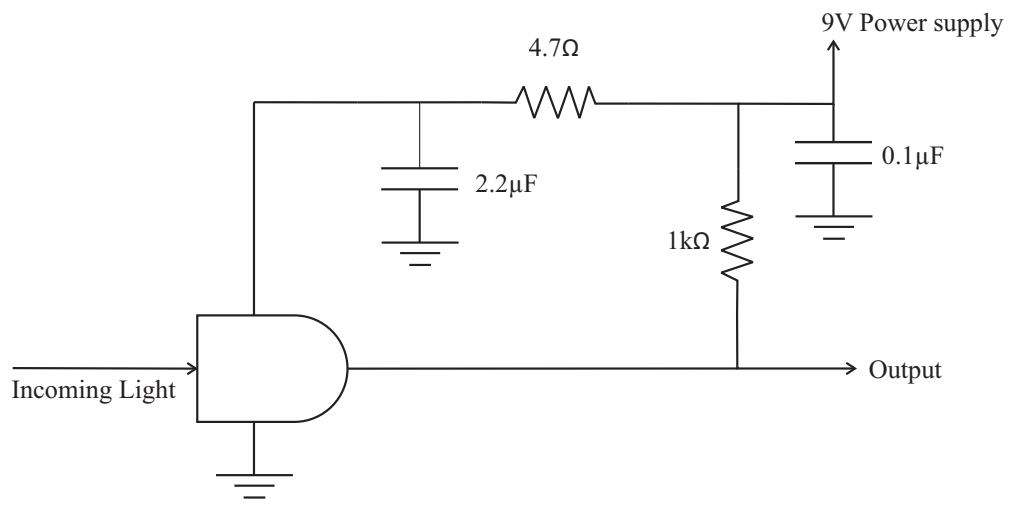

Figure A-4: Photodiode triggering circuit

\section{Understanding the image}

It is very important to understand how to read a schlieren picture. The photograph enhances the contrast of the diffraction caused by a change in density in the medium. Light rays are bent towards the higher density region as it is implied by the GladstoneDale linear relationship between refractive index and density $(\mathrm{n}-1=\mathrm{k})$. Fig. A-5 shows a spherical shockwave generated by the discharge of a high voltage capacitor across a gap switch as well as the knife edge configuration that was used in this picture. It is very clear that the region upstream is unaffected and therefore only shows the gray-scale background associated with the knife edge. The second very important thing to notice is the fact the shockwave is half bright and half dark. Earlier we mentioned that depending on the knife edge orientation we can observe different density gradient. In this case the knife edge was vertical allowing us to see horizontal density gradients. Following the image from left to right we notice that the density gradient across the left part of the shock increases in magnitude and therefore so does the refracting index. This leads to a ray deflection away from the knife edge and is therefore appearing white on the picture. However if 
we continue to move from left to right across the right portion of the shock we now have a negative density gradient as density is smaller in the upstream region. This leads to a decreasing refracting index and thus the light is deflected towards the knife edge which leads to a dark spot. Therefore for the same shockwave we see both a dark and bright region that have the same density gradients across them.

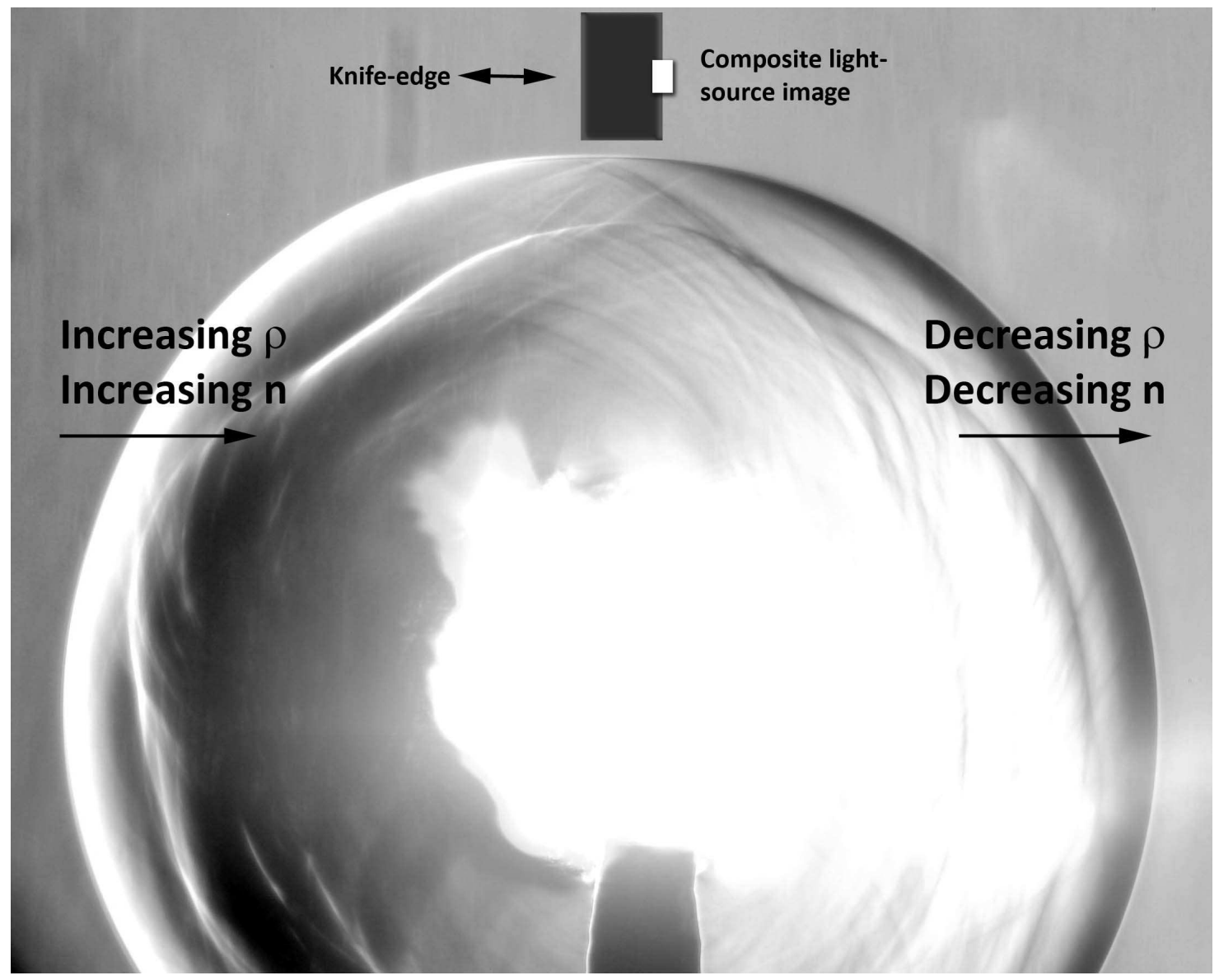

Figure A-5: Spherical shock generated by high voltage discharge obtained from Grondin [11] 


\section{APPENDIX B \\ Theory}

Consider the oblique detonation wave shown in Fig. B-1. We can express the conservation equations as follows:

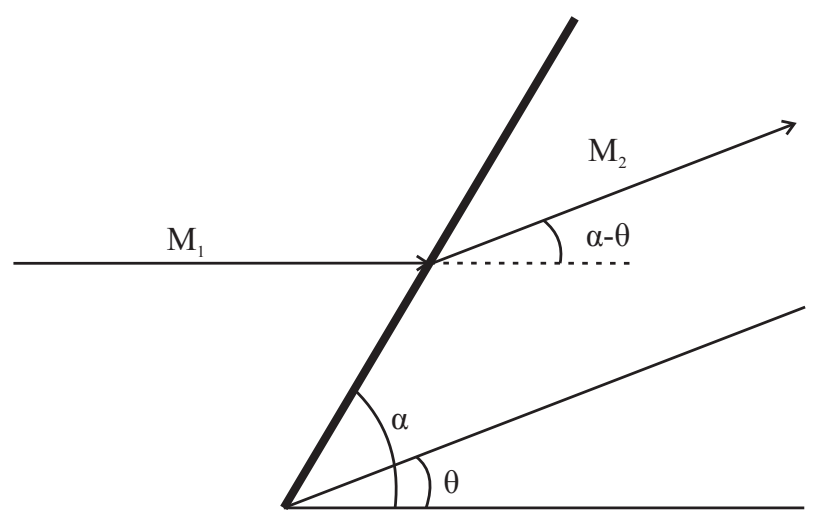

Figure B-1: Oblique Detonation Diagram

Mass:

$$
\rho_{1} u_{1} \sin (\alpha)=\rho_{2} u_{2} \sin (\alpha-\theta)
$$

Normal momemtum:

$$
P_{1}+\rho_{1} u_{1}^{2} \sin ^{2} \alpha=P_{2}+\rho_{2} u_{2}^{2} \sin ^{2}(\alpha-\theta)
$$

Tangential momemtum:

$$
\rho_{1} u_{1}^{2} \sin \alpha \cos \alpha=\rho_{2} u_{2}^{2} \sin (\alpha-\theta) \cos (\alpha-\theta)
$$


Energy:

$$
C_{p 1} T_{1}+\frac{u_{1}^{2}}{2}+Q=C_{p 2} T_{2}+\frac{u_{2}^{2}}{2}
$$

Equation of state:

$$
P=\rho R T
$$

We thus have 5 equations for the 5 unknowns $\left(\rho_{2}, \mathrm{u}_{2}, \mathrm{P}_{2}, \mathrm{~T}_{2}\right.$ and $\left.\theta\right)$. Under the assumption that $\mathrm{C}_{p 1}=\mathrm{C}_{p 2}$ the equations can be reduced in term for the density ratios as demonstrated by Gross [12]. We have:

$\frac{\rho_{1}}{\rho_{2}}=\frac{1+\gamma M_{1}^{2} \sin ^{2} \alpha \pm \sqrt{\left[1+\gamma M_{1}^{2} \sin ^{2} \alpha\right]^{2}-(\gamma+1) M_{1}^{2} \sin ^{2} \alpha\left[2+\left(2 Q / C_{p} T_{1}\right)+(\gamma-1) M_{1}^{2} \sin ^{2} \alpha\right]}}{(\gamma+1) M_{1}^{2} \sin ^{2} \alpha}$

Two real states that can be obtained from the above which represent the weak and strong detonation solution. The CJ solution is recovered, if the radical is equal to zero as performed by Gross in his paper. The remaining variables can be found using the ratio of densities:

$$
\begin{aligned}
\frac{V_{2}}{V_{1}}= & \sqrt{1-\sin ^{2} \alpha\left[1-\left(\rho_{1} / \rho_{2}\right)^{2}\right]} \\
\frac{P_{2}}{P_{1}}= & 1+\gamma M_{1}^{2} \sin ^{2} \alpha\left[1-\rho_{1} / \rho_{2}\right] \\
& \frac{T_{2}}{T_{1}}=\left(P_{2} / P_{1}\right)\left(\rho_{1} / \rho_{2}\right) \\
\theta= & \alpha-\cos ^{-1}\left[\left(V_{2} / V_{1}\right) \cos \alpha\right]
\end{aligned}
$$

Q can be obtained using the following equation:

$$
M_{c j}=\sqrt{1+(\gamma+1) \frac{Q}{C_{p} T_{1}}+\sqrt{\left(1+(\gamma+1) \frac{Q}{C_{p} T_{1}}\right)^{2}-1}}
$$

Where $\mathbf{M}_{c j}$ is obtained from CEA or STANJAN for a given mixture. 
Three-Shock theory can be used to solve for the triple point trajectory $\chi$ as well as the critical wedge angle for transition between regular and Mach reflection. As mentioned earlier we change the frame of reference to move with the triple point and solve for the flow conditions in its vicinity by using two contraints introduced by Law and Glass (1971) [19]:

1. The Mach Stem must be normal to the wedge as well as be straight.

2. The pressure and flow deflection must be the same across the slipstream between region 2 and 3.

Of course the first condition violates the fact that the Mach stem must be curved as was pointed out earlier, however we are only interested in the flow very near the triple point this assumption can be used. Fig. B-2 shows the flow configuration. From geometry we obtain:

$\alpha_{1}=90^{\circ}-\theta_{w^{-}} \chi$

$\alpha_{3}=90^{\circ}-\chi$

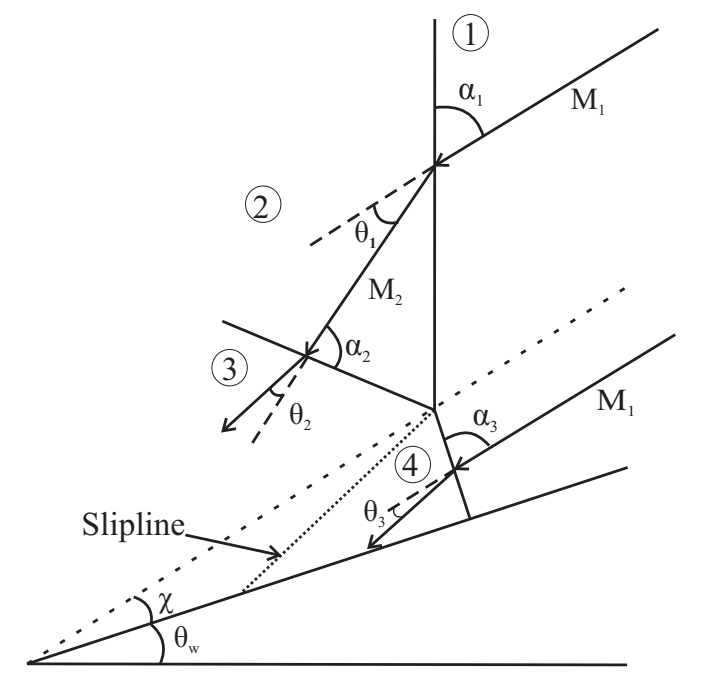

Figure B-2: Three-Shock configuration from the triple point 
One can proceed as follow to solve the problem. First guess a $\chi$ for a known value of $\theta_{w}$. Then based on the geometry $\alpha_{1}$, and $\alpha_{3}$ are known. We require that the incident wave is a wave moving at the $\mathrm{CJ}$ velocity, thus the normal component of $\mathbf{M}_{1}$ must be $\mathbf{M}_{c j}$, and hence once again from geometry $\mathrm{M}_{1}$ is known. We can now solve for the flow conditions between region 1 and 2 and between region 1 and 4 .

\section{Region 1 to 2}

This is an oblique detonation with wave angle $\alpha_{1}$ and known $\mathrm{M}_{1}$. The detonation is considered to be overdriven because the normal component is $\mathrm{M} c j$, therefore the overdriven solution for the density ratios obtained above must be used.

Thus :

$$
\begin{gathered}
\frac{\rho_{1}}{\rho_{2}}=\frac{1+\gamma M_{1}^{2} \sin ^{2} \alpha_{1}-\sqrt{\left[1+\gamma M_{1}^{2} \sin ^{2} \alpha_{1}\right]^{2}-(\gamma+1) M_{1}^{2} \sin ^{2} \alpha_{1}\left[2+Q / C_{p} T_{1}+(\gamma-1) M_{1}^{2} \sin ^{2} \alpha_{1}\right]}}{(\gamma+1) M_{1}^{2} \sin ^{2} \alpha_{1}} \\
\frac{V_{2}}{V_{1}}=\sqrt{1-\sin ^{2} \alpha_{1}\left[1-\left(\rho_{1} / \rho_{2}\right)^{2}\right]} \\
\frac{P_{2}}{P_{1}}=1+\gamma M_{1}^{2} \sin ^{2} \alpha_{1}\left[1-\rho_{1} / \rho_{2}\right] \\
\frac{T_{2}}{T_{1}}=\left(P_{2} / P_{1}\right)\left(\rho_{1} / \rho_{2}\right) \\
\frac{M_{2}}{M_{1}}=\left(V_{2} / V_{1}\right) \sqrt{\left(T_{1} / T_{2}\right)} \\
\theta_{1}=\alpha_{1}-\cos ^{-1}\left[\left(V_{2} / V_{1}\right) \cos \alpha_{1}\right]
\end{gathered}
$$

\section{Region 1 to 4}

Similarly, this is an oblique detonation wave problem with $\alpha_{3}$ and known $\mathbf{M}_{1}$, and the 
detonation wave is still overdriven therefore:

$$
\begin{gathered}
\frac{\rho_{1}}{\rho_{4}}=\frac{1+\gamma M_{1}^{2} \sin ^{2} \alpha_{3}-\sqrt{\left[1+\gamma M_{1}^{2} \sin ^{2} \alpha_{3}\right]^{2}-(\gamma+1) M_{1}^{2} \sin ^{2} \alpha_{3}\left[2+Q / C_{p} T_{1}+(\gamma-1) M_{1}^{2} \sin ^{2} \alpha_{3}\right]}}{(\gamma+1) M_{1}^{2} \sin ^{2} \alpha_{3}} \\
\frac{V_{4}}{V_{1}}=\sqrt{1-\sin ^{2} \alpha_{3}\left[1-\left(\rho_{1} / \rho_{4}\right)^{2}\right]} \\
\frac{P_{4}}{P_{1}}=1+\gamma M_{1}^{2} \sin ^{2} \alpha_{3}\left[1-\rho_{1} / \rho_{4}\right] \\
\frac{T_{4}}{T_{1}}=\left(P_{4} / P_{1}\right)\left(\rho_{1} / \rho_{4}\right) \\
\theta_{3}=\alpha_{3}-\cos ^{-1}\left[\left(V_{4} / V_{1}\right) \cos \alpha_{3}\right]
\end{gathered}
$$

Since we require that the flow in region 3 and 4 be parallel we must have $\theta_{3}=\theta_{1}+$ $\theta_{2}$ and hence $\theta_{2}$ is known and we can solve for the quantities in the final region.

\section{Region 2 to 3}

In this case the problem is one of an oblique shockwave $(Q=0)$ but this time the wave angle $\alpha_{2}$ is unknown but the deflection $\theta_{2}$ is.

A value for $\alpha_{2}$ between $90 \mathrm{deg}$ and $\sin ^{-1}\left(1 / M_{2}\right)$ is guessed and we obtain:

$$
\begin{gathered}
\frac{\rho_{2}}{\rho_{3}}=\frac{1+\gamma M_{2}^{2} \sin ^{2} \alpha_{2}-\sqrt{\left[1+\gamma M_{2}^{2} \sin ^{2} \alpha_{2}\right]^{2}-(\gamma+1) M_{2}^{2} \sin ^{2} \alpha_{2}\left[2+(\gamma-1) M_{2}^{2} \sin ^{2} \alpha_{2}\right]}}{(\gamma+1) M_{2}^{2} \sin ^{2} \alpha_{2}} \\
\frac{V_{3}}{V_{2}}=\sqrt{1-\sin ^{2} \alpha_{2}\left[1-\left(\rho_{2} / \rho_{3}\right)^{2}\right]} \\
\frac{P_{3}}{P_{2}}=1+\gamma M_{2}^{2} \sin ^{2} \alpha_{2}\left[1-\rho_{2} / \rho_{3}\right] \\
\frac{T_{3}}{T_{2}}=\left(P_{3} / P_{2}\right)\left(\rho_{2} / \rho_{3}\right)
\end{gathered}
$$




$$
\theta_{2}=\alpha_{2}-\cos ^{-1}\left[\left(V_{3} / V_{2}\right) \cos \alpha_{2}\right]
$$

If $\theta_{2}$ obtained using the equations is the same as the one obtained from $\theta_{3}=\theta_{1}+\theta_{2}$ then the process is stopped.

The pressure in region 3 and 4 can now be compared. If they are not equal then the whole process is repeated with a new value of $\chi$ until they match. This $\chi$ is the triple point trajectory. When $\chi$ cannot be solved for a given $\theta_{W}$ then regular reflection occurs.

The process above is for the reactive case of thin discontinuity detonation waves. The unreactive case (shockwaves) uses the same procedure, however Q is set to 0 for all regions. 